\title{
The OphthaCoil : a new vehicle for the delivery of drugs to the eye
}

Citation for published version (APA):

Pijls, R. T. (2007). The OphthaCoil : a new vehicle for the delivery of drugs to the eye. [Doctoral Thesis, Maastricht University]. Universiteit Maastricht. https://doi.org/10.26481/dis.20071217rp

Document status and date:

Published: 01/01/2007

DOI:

10.26481/dis.20071217rp

Document Version:

Publisher's PDF, also known as Version of record

\section{Please check the document version of this publication:}

- A submitted manuscript is the version of the article upon submission and before peer-review. There can be important differences between the submitted version and the official published version of record.

People interested in the research are advised to contact the author for the final version of the publication, or visit the DOI to the publisher's website.

- The final author version and the galley proof are versions of the publication after peer review.

- The final published version features the final layout of the paper including the volume, issue and page numbers.

Link to publication

\footnotetext{
General rights rights.

- You may freely distribute the URL identifying the publication in the public portal. please follow below link for the End User Agreement:

www.umlib.nl/taverne-license

Take down policy

If you believe that this document breaches copyright please contact us at:

repository@maastrichtuniversity.nl

providing details and we will investigate your claim.
}

Copyright and moral rights for the publications made accessible in the public portal are retained by the authors and/or other copyright owners and it is a condition of accessing publications that users recognise and abide by the legal requirements associated with these

- Users may download and print one copy of any publication from the public portal for the purpose of private study or research.

- You may not further distribute the material or use it for any profit-making activity or commercial gain

If the publication is distributed under the terms of Article $25 \mathrm{fa}$ of the Dutch Copyright Act, indicated by the "Taverne" license above, 


\section{The OphthaCoil}

a new vehicle for the delivery of drugs to the eye 
The OphthaCoil: a new vehicle for the delivery of drugs to the eye

ISBN 978-90-8570-296-2

Printed by: Wöhrmann Print Service, Zutphen, the Netherlands

Cover design: K. de Boer (AzM Oogheelkunde) and Joris Kockelkoren

Copyright (C R.T. Pijls, Maastricht 2007 


\section{The OphthaCoil}

\section{a new vehicle for the delivery of drugs to the eye}

\section{PROEFSCHRIFT}

ter verkrijging van de graad van doctor

aan de Universiteit Maastricht,

op gezag van de Rector Magnificus,

Prof. mr. G.P.M.F. Mols,

volgens het besluit van het College van Decanen,

in het openbaar te verdedigen op

maandag 17 december 2007 om 12.00 uur

door

\section{Rachel Theodora Pijls}

Geboren op 10 september 1980 te Heerlen 


\section{Promotor}

Prof. dr. ir. L.H. Koole

\section{Copromotores}

Dr. R.M.M.A. Nuijts

Dr. A.A. Dias (DSM Research)

\section{Beoordelingscommissie}

Prof. dr. F. Hendrikse (voorzitter)

Prof. dr. M.P. van Dieijen-Visser

Prof. dr. J.M.M. Hooymans (Rijksuniversiteit Groningen)

Prof. dr. H.C.J. Ottenheijm

Prof. dr. H.A.J. Struijker Boudier

This thesis was performed in collaboration with and financially supported by DSM Research, Geleen, the Netherlands. 


\section{CONTENTS}

$\begin{array}{lll}\text { Chapter } 1 & \text { General Introduction } & 7\end{array}$

$\begin{array}{lll}\text { Chapter } 2 \text { Flexible coils with a drug-releasing hydrophilic coating: a new } & 29\end{array}$

platform for controlled delivery of drugs to the eye?

Chapter 3 Studies on a new device for drug delivery to the eye 45

Chapter 4 Preliminary study on the patient compliance of the OphthaCoil 59

$\begin{array}{lll}\text { Chapter } 5 \text { Increasing the capacity of the OphthaCoil using drug-loaded } & 67\end{array}$ microspheres

Chapter 6 Pradofloxacin release from the OphthaCoil 85

Chapter 7 The OphthaCoil for treatment of infections or pre-operative 99 delivery of drugs to the eye

Chapter 8 Highly porous UHMWPE-films as a matrix for controlled drug 115 delivery

$\begin{array}{lll}\text { Chapter } 9 & \text { General Discussion } & 137\end{array}$

$\begin{array}{lll}\text { Appendix I Title page patent } & 143\end{array}$

$\begin{array}{ll}\text { Summary } & 145\end{array}$

$\begin{array}{ll}\text { Samenvatting } & 150\end{array}$

$\begin{array}{ll}\text { List of Publications } & 155\end{array}$

$\begin{array}{ll}\text { Curriculum vitae } & 157\end{array}$

$\begin{array}{lc}\text { Dankwoord } & 158\end{array}$ 


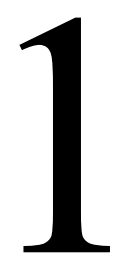

General Introduction 


\subsection{Preface}

The eye, our window to the outside world, allows us to view the beauties of nature. It is composed of tissues that require continuous nourishment and use metabolic pathways appropriate to their unique needs [1]. The eyebrows, tear fluid and eyelashes protect the eye from external influences, like sunlight, sweat and dust. Despite this protection, lots of things can happen to the eye, such as insufficient amount of tear fluid (dry eye syndrome), irritation/infections, or illness (like diabetes mellitus) and aging can induce ocular disorders. Most diseases can be treated by the delivery of drugs. Despite the accessibility of the anterior surface of the eye, efficient delivery of drugs to treat the various ocular disorders is a challenge $[2,3]$. The eye has two compartments: an anterior chamber, present between the cornea and the ocular lens, and a vitreous cavity behind the lens. There is no vascular connection between these chambers. Besides the isolated structure, other protection mechanisms of the eye, like tissue barriers (e.g. cornea, sclera and conjunctiva), or tear turnover and blinking make it more difficult to deliver drugs to the therapeutic targets [4-6].

The selection of the route of administration depends primarily on the target tissue. Traditional topical ocular and sub-conjunctival administrations are used for anterior targets and intravitreal administration for posterior targets [7, 8]. Our focus area is the anterior route, which is important in the treatment of diseases such as inflammations, infections, and glaucoma [9]. Topical application of drugs via solutions, ointments or suspensions account for nearly $90 \%$ of all available ophthalmic formulations $[10,11]$. Despite the fact that these formulations are very common, the pharmacokinetic characteristics of these formulations, i.e. pulsed administrations of high drug concentrations, may be undesirable.

In this thesis, the development of a new ocular device for the delivery of drugs to the tear film is described. The insert is called the OphthaCoil. It is based on a drug-loaded hydrogel coated onto a metallic substrate. The device is placed under the eyelid, where the hydrogel coating takes up tear fluid, swells, and releases its entrapped drug gradually to the tear film.

In this chapter, first the anatomy of the eye is described, where several ocular tissues are highlighted. The possible causes of visual impairment are then classified as well as the current methods for the topical delivery of ocular drugs. Finally, the outline of this thesis is described. 


\subsection{Anatomy of the eye}

The cornea is considered to be the main route by which topically applied drugs enter the eye. Another potential route of ocular drug absorption is across the bulbar conjunctiva and sclera into the uveal tract and vitreous humor [12], see Figure 1.1. Absorption is defined as the process by which a drug enters the aqueous humor. The rate and extent of absorption (defined as bioavailability [13]) refer to how quickly and extensively the administered dose enters the aqueous humor [14].

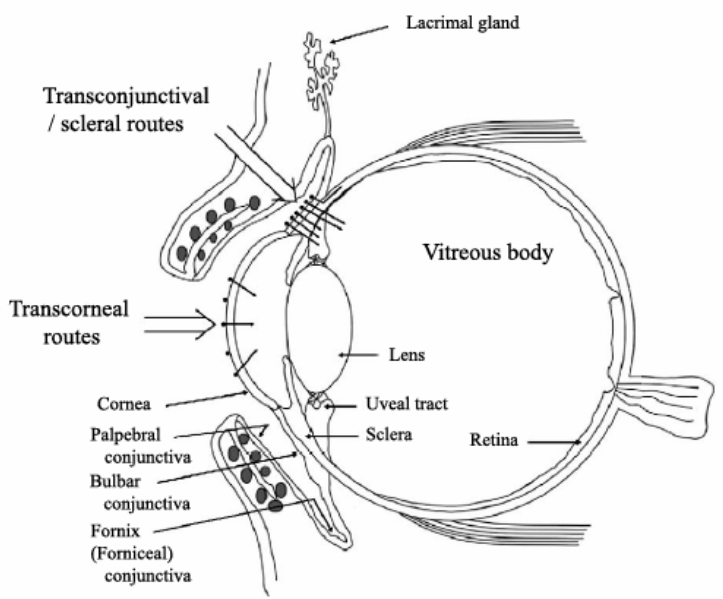

Figure 1.1. Cross-section of the eye [7].

\subsubsection{The cornea}

Although the human cornea consists of five layers, only three layers provide significant barrier resistance to absorption into the aqueous humor $[14,15]$, i.e. the epithelium, the stroma and the single layer of endothelium, see Figure 1.2 [16]. The epithelium is $60-65 \mathrm{~nm}$ thick [17], and provides for most drugs the highest resistance to penetration [14]. The aqueous corneal stroma comprises approximately $90 \%$ of the corneal thickness and is composed of layers of parallel lamellae and holds $75-80 \%$ water by net weight. The single layer endothelium serves as a barrier between the hydrophilic aqueous stroma of the cornea and the aqueous humor of the anterior chamber [17]. The special arrangement of cells, lack of vascularity, and regularity and smoothness of the epithelium make the cornea transparent [17]. 


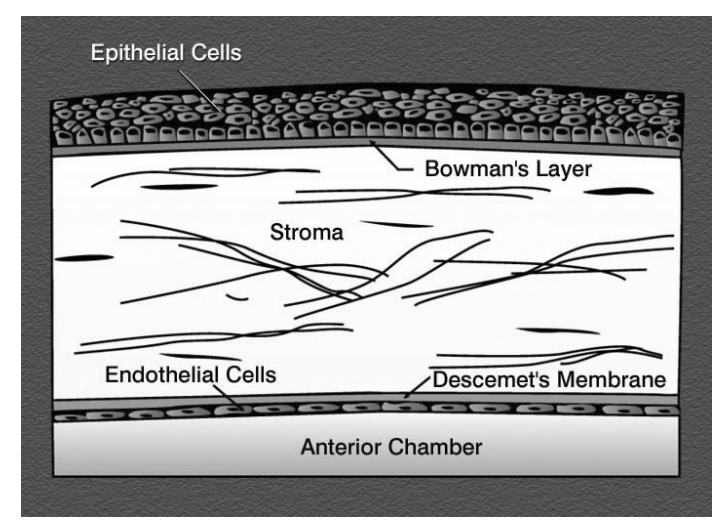

Figure 1.2. The different layers of the cornea [16].

\subsubsection{The conjunctiva}

The conjunctiva is a thin transparent mucous epithelial barrier, lining on the inside of the eyelids, and covering the anterior one-third of the eyeball. The respective portion of conjunctiva is referred to as the palpebral and bulbar conjunctiva. The joining area is referred to as the fornix, see Figure 1.1. The conjunctiva is composed of two layers: an outer epithelium and its underlying stroma [7]. The epithelium is from 2 to 10 cell layers thick, and the epithelium cells contain numerous mucus producing goblet cells [17, 18]. The stroma, containing structural and cellular elements, including nerves, lymphatics and blood vessels, is loosely attached to the underlying sclera [7].

\subsubsection{The tear film}

The exposed surface of the eye includes conjunctiva and cornea and is covered with the tear film. The conjunctiva contributes to the formation of the tear film by secreting substantial electrolytes, fluid, and mucins [7]. The tear film consists of three layers, an outer lipid layer, a middle aqueous layer, and an inner mucous layer [18]. The tear film fulfils several important functions in the eye; it (i) forms and maintains a smooth refracting surface over the cornea, (ii) maintains a moist environment for the epithelial cells of the cornea and conjunctiva, (iii) has bactericidal properties, (iv) lubricates the eyelids, (v) transports metabolic products (primarily oxygen and carbon dioxide) to and from the epithelial cells and cornea, (vi) provides a pathway for white blood cells in case of injury and (vii) dilutes and washes away noxious stimuli [19]. Under normal conditions, the tear volume is $7-9 \mu \mathrm{L}$ in humans with a turnover rate of $0.5-2.2$ $\mu \mathrm{L} / \min [14,20]$. Most of the volume resides in the conjunctival sacs with approximately $1 \mu \mathrm{L}$ covering the cornea [2]. 


\subsubsection{The lens}

The lens is an optically clear structure located behind the iris, and in front of the vitreous body and the retina [21]. The lens is bathed on one side by aqueous humor and supported on the other side by vitreous humor. It has no blood supply but is metabolically active. It gets nutrients from the aqueous humor and eliminates waste into the aqueous humor. The structure of the lens comprises a monolayer of epithelial cells that covers elongated and precisely aligned fiber cells [22].

The lens is responsible for fine-tuning the image that is projected on the retina. To perform its function, the lens must be transparent and have a high refractive index. The transparency of the lens depends on the organization of the cells and of the distribution of the proteins (crystallins) within. The refractive properties of the lens are the result of the high concentration of these proteins and the curvature of the lens surfaces [23].

\subsubsection{The sclera}

The human sclera is an elastic and microporous tissue composed of proteoglycans and closely packed collagen fibrils, containing approximately $70 \%$ water [24, 25]. Scleral collagen fibers, $90 \%$ of which are collagen type I, constitute $75 \%$ to $80 \%$ of the dry weight of the sclera. The sclera, like the cornea, is essentially avascular except for the superficial vessels of the episclera and the intrascleral vascular plexus located just posterior to the limbus [26].

The sclera performs several important functions essential for the visual integrity of the eye. Primarily, the sclera provides a firm substrate for the delicate intraocular contents and protects them from injury. In addition, it facilitates rotation of the eyeball without significant distortion to nearly $180^{\circ}$ by powerful muscles. The shape of the eye is, in part, maintained by the presence of the intraocular contents and the intraocular pressure [27].

\subsubsection{The aqueous chamber and the vitreous body}

The aqueous humor flows around the lens and through the pupil into the anterior chamber. The circulating aqueous humor nourishes the cornea and the lens, and it removes end products of metabolism from them [1]. It provides a transparent and colorless medium with a refractive index 1.333 between the cornea and the lens, thus constituting an important component of the eye's optical system [28].

The vitreous body is a transparent gel that occupies the vitreous cavity. The collagenous or gelatinous mass helps maintain the shape of the eye, while allowing it to remain flexible [1]. It has an almost spherical appearance, except for the anterior part, which is concave corresponding to the presence of the crystalline lens [29]. 


\subsubsection{The retina}

Light enters the eye through the lens and is refracted to the back of the eye onto the retina. Important vision cells in the retina, the photoreceptor cells, convert the focused light, into an electrical signal which then travels to the brain through the optic nerve. In the brain that electrical signal is experienced as vision [30]. There are two types of photoreceptor cells: rod cells, responsible for vision in dim light, and cone cells, responsible for color vision.

The macula lutea (yellow spot) is an area located in the center of the retina, which is approximately $5.5 \mathrm{~mm}$ in diameter. With help of the focussing lens, light narrows to a point on the macula, the fovea. This is the primary location of the cones. The place on the retina where the optic nerve leads back into the brain, is the blind spot. The retina has no light-sensitive rods or cones at this point. This means that an object in the field of the blind spot becomes invisible [31].

\subsubsection{The pupil}

The pupil acts as an objective indicator of the amount of light transduction by the visual system [32]. The major functions of the pupil are listed here: (i) pupil movement, in response to changing light intensity, assists in optimizing retinal illumination to maximize vision. In dim light, dilation of the pupil provides an immediate means for maximizing the number of photons reaching the retina; (ii) the diameter of the pupil can also contribute to improving the image quality of the retina when the steady-state pupil diameter is small; and (iii) a small pupil increases the depth of focus of the eye's optical system. When a person views objects at close distance, the pupil contraction helps to bring objects into better focus by increasing the depth of focus [32].

\subsection{Visual impairment}

The World Health Organization (WHO) states that an estimated 37 million people worldwide are blind. Seventy-five percent of this blindness is treatable or preventable. Without proper interventions the number of blind people will increase to 75 million by 2020 [33]. The most

important causes of visual impairment (i.e. blindness and low vision) in the world today can be divided into several categories [34], which will be discussed here. 


\subsubsection{Refractive error}

Refractive error has been identified as the leading cause of visual impairment in the developed world [34]. The impact of myopia (near-sightedness) and hyperopia (far-sightedness) can generally be undone by the use of spectacles, contact lenses, or refractive surgery. When refractive error is corrected, vision may be lost due to bacterial keratitis associated with contact lens wear or complications from refractive surgery [34].

\subsubsection{Age-related causes of blindness}

Cataract is the leading cause of blindness worldwide and may concern as many as 17 million people [34-38]. Cataract is an opacification of the lens that causes the loss of transparency and/or scattering of light entering the eye [35]. The transparency of the lens can be destroyed by the disruption of the precise organization of the lens fibre cells or by damage to the proteins within them [23]. In the last decades, cataract surgery underwent remarkable technical refinement. The current state-of-the-art is sutureless phaco-emulsification surgery with foldable intraocular lens implantation [39]. In hospitals in the Netherlands, $75 \%$ of all eye operations are cataract surgeries [40]. An important issue regarding cataract surgery is the risk of endophthalmitis (i.e. inflammation of the interior ocular structures). Currently, patients are pretreated with antibiotics to decrease this risk of inflammation. Nevertheless, the incidence of endophthalmitis associated with cataract surgery has increased over the last decade [39].

Age-related macular degeneration (AMD) is the most common cause of irreversible blindness and visual impairment in individuals of 65 years and older in the developed world [35, 41, 42]. AMD is a degenerative disease involving damage and death of cells of the macula, the region of the retina responsible for central vision [43]. Although AMD is currently incurable, advances in the understanding of its pathophysiology have led to significant therapeutic innovation over the past decade. Vascular endothelial growth factor (VEGF) in particular, contributes to angiogenesis, and anti-VEGF pharmacotherapy forms an important step forward in the treatment of AMD [43].

Glaucoma is a progressive optic neuropathy characterized by functional and structural impairment of ocular tissues. It leads to visual field defects and, ultimately, blindness [35]. The structural changes in the tissues lead to elevated intraocular pressure. Systemic hypertension is suspected to increase the risk of the development and progression of glaucoma [44]. Glaucoma is not a single condition, but a heterogeneous group of diseases that are classified as primary or secondary, open- or closed-angle, according to age of onset and degree of ocular hypertension 
[35]. Medical therapy is generally focussed on decreasing aqueous production or increasing aqueous outflow [45].

\subsubsection{Infectious causes of blindness}

Systemic inflammatory diseases commonly affect the sclera, cornea, retina, and orbit, and can pose a serious threat to sight [46]. Trachoma is a chronic keratoconjunctivitis, which is caused by repeated infection with Chlamydia trachomatis. Repeated infections lead to conjunctival scarring and deformation of the lid margin may cause the eyelashes to turn inward (entropion) and repeatedly rub against the cornea (trichiasis). The catastrophic outcome is corneal opacification and, ultimately, blindness. It accounts for $15 \%$ of cases of blindness worldwide $[47,48]$. Circumstantial evidence links this disease specifically to poor hygiene and inadequate access to clean water [48]. Nowadays in the developed world, the risk of developing bacterial keratitis is increasing, because of contact lens wear [49-51].

\subsubsection{Nutritional and metabolic causes of blindness}

Diabetes mellitus has many manifestations in the eye, of which cataract and diabetic retinopathy (DR) are the most significant cause of visual impairment. People with diabetes are 25 times more likely to become blind than the general population [52]. DR is a leading cause of blindness among adults younger than 40 years in the developed world, but it affects older individuals as well [34]. The treatment of DR is to some degree limited by the difficulty of delivering drugs to the retina, the target tissue in the posterior eye. Traditional treatment (i.e. systemic or topical drug delivery) does not result in therapeutic drug levels in the posterior tissues. Delivery of drugs through the permeable sclera or the use of less invasive devices would be safer and could provide a more effective retinal dose [53]. Clinical trials have documented the benefit of early laser treatment in preventing blindness among individuals with DR [34].

\subsubsection{Trauma}

Endophthalmitis is not only a risk factor after ocular surgery, but is also a serious complication of penetrating ocular trauma [54]. Risk factors of developing endophthalmitis after open globe injuries are dirty wounds, opening of the lens capsule and delayed primary repair [55]. The annual cost of ocular trauma in the US is estimated at \$175-200 million for hospital care alone [34]. 


\subsubsection{Other eye conditions}

The tear film is crucial to ocular surface health. Dysfunction may result in the dry eye syndrome [56]. Dry eye is defined as a disorder causing ocular discomfort and ocular surface damage, due to tear deficiency or excessive tear evaporation [56, 57]. Artificial tears represent the main treatment for dry eyes and are arguably the most frequently used local ophthalmic medication $[58,59]$.

\subsection{Drug permeability of ocular barriers}

In order to effectively reach therapeutic targets in the eye, drugs must penetrate across the eye's barriers, i.e. the cornea, sclera, conjunctiva [4]. The permeability of drugs across these tissues is influenced by various factors, such as lipophilicity, molecular weight and charge [60]. The permeability of these barriers will now be discussed.

\subsubsection{Cornea}

The permeability of the cornea is dependent on the octanol-water distribution coefficient of the transported molecule. The permeability increases with increasing distribution coefficient. This means that lipophilic drugs permeate faster and to a greater extent than hydrophilic drugs $[4$, $60]$.

For molecules smaller than $10 \AA$ (i.e. with a molecular weight smaller than approximately 1500 $\mathrm{Da}$ ), there is no evidence that the molecular radius influences the corneal permeability. Molecules with a radius larger than $10 \AA$ generally can not cross the cornea at measurable rates. This is due to the pore size in the intercellular space [60-62].

The pores of the corneal epithelium are negatively charged at physiological $\mathrm{pH}$, which means that negatively charged drugs permeate slower than positively charged or neutral drugs. However, positively charged drugs may decrease the permeability as well, due to ionic interaction [60].

After crossing the cornea, the drug diffuses into the aqueous humor. Topically applied drugs that enter the eye via the cornea cannot reach the retina and vitreous at sufficient therapeutic concentrations [60].

\subsubsection{Conjunctiva}

In rabbits, the conjunctival epithelium has 2 times larger pores and a 16 times higher pore density than the corneal epithelium. This results in a 15 - to 25 -fold higher permeability in 
comparison to the cornea $[12,60]$. Also, the surface area of the conjunctiva is 17 times larger than that of the cornea. This may contribute to the greater absorption of hydrophilic drugs via the conjunctiva, as the conjunctiva have more tight junctions as the cornea $[7,63]$.

However, data on the permeability of the conjunctiva is quite limited $[4,7]$. Horibe et al. found that the permeability of hydrophilic drugs decreases with increased molecular weight [64]. Because of the larger pores compared to the cornea, hydrophilic drugs with molecular weights less than 20,000 - 40,000 Da can permeate the conjunctiva [62, 64]. This makes the conjunctiva permeable for small peptides and oligonucleotides [12].

\subsubsection{Sclera}

The sclera is about 10 times more permeable than the cornea, and half as permeable as the conjunctiva [60]. This, in combination with the large surface area of the sclera, has stimulated transscleral drug delivery, especially for drugs that need to be administered to the back of the eye (i.e. posterior drug delivery) $[4,65,66]$.

The permeability of the sclera shows no dependence on lipophilicity, but is strongly dependent on the molecular radius. The permeability increases with decreasing radius. However, there is no evidence that the distribution coefficient influences the permeability [4].

\subsection{Topical administration of ocular drugs}

A considerable amount of effort has been made in ophthalmic drug delivery since the 1970s. The various approaches can be divided into two main categories: bioavailability improvement and controlled release of drug delivery [67]. The most common methods of topical ocular drug delivery will be discussed.

\subsubsection{Eye drops (solutions and suspensions)}

Eye drops are the most used dosage form by ocular route [68], but this method has several disadvantages. Using eye drops, the actual dose absorbed by the eye is not known. The volume of tear fluid in the eye is approximately 7-9 $\mu \mathrm{L}$ [20]. The volume delivered by most commercial ophthalmic eye drop dispensers is $50 \mu \mathrm{L}$. It has been estimated that the human eye can hold approximately $30 \mu \mathrm{L}$ without overflow or spillage at the outer angle [2]. This means that a large proportion of drug is wasted due to the administration of such an excess volume. Another disadvantage is the tear turnover. Secretion and drainage of tears occur at a rate of approximately $1 \mu \mathrm{L} / \mathrm{min}$, which equates to a $16 \%$ turnover of the tear film per minute [2]. 
These and other mechanisms, like tear evaporation and non-productive absorption, contribute to the fact that the contact time of an applied dose is less than two minutes, with the result that even less than $5 \%$ of an applied dose actually reaches the intraocular tissues and more than $50 \%$ is systemically absorbed $[3,5,7,62,67-70]$.

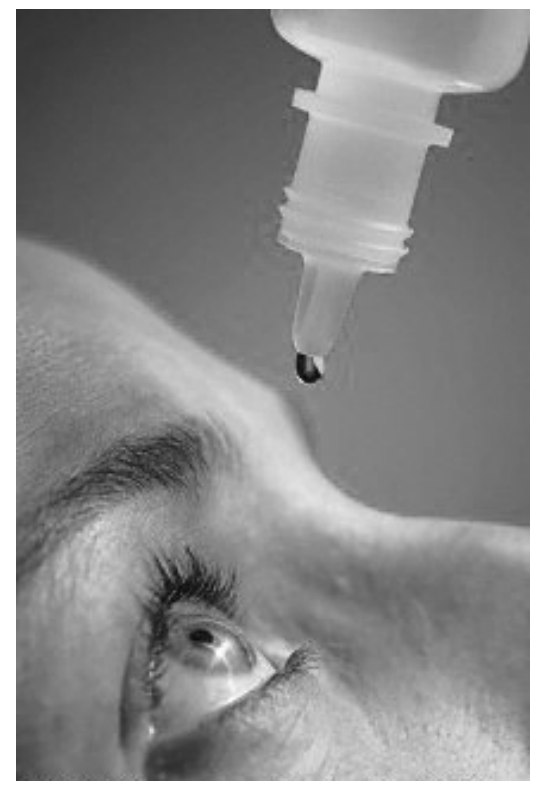

Figure 1.3. The administration of an eye drop [72].

Therefore, pulsed administrations of high drug concentrations are necessary, but this is highly undesirable. The initial high drug concentration found in tears, followed by a rapid decline, poses a potential risk of toxicity [67]. The preservatives present in eye drops may cause some problems as well. The most frequent used preservative, benzalkonium chloride, has toxic side effects [71]. The long-term use of eye drops has consistently been reported to induce inflammatory ocular surface changes, causing progressive ocular discomfort upon instillation, tear film instability, and corneal surface impairment [71].

Besides technical problems, also practical issues arise, like the administration of the drop in the eye. Patients find it difficult to instil a drop adequately, see Figure $1.3[72,73]$. These problems arise in both human and veterinary medicine. In human ophthalmology, especially children and elderly have problems with the administration of eye drops. In the Netherlands, relatives or people from domiciliary care have to visit elderly frequently only to administer the required eye drops, with associated financial burden to health care.

In animals, the main diseases are similar and exhibit similar pathologies to those of the human eye. Ocular inflammation is one of the most common eye disorders in animals, but the 
administration of drugs to animals, like dogs, cats and horses, is often difficult, particularly when repeated applications are required. In horses, the orbicularis oculi muscle is so powerful that it is often impossible to retract the upper eyelid [74]. Also, it is difficult to tilt the head of the horse to apply a drop of solution without spilling the contents of the bottle. In cats antiviral agents must be administered topically every $1-2 \mathrm{~h}$ in order to obtain a therapeutic effect to treat herpes keratitis (inflammation of the cornea). Frequent installations are often inconvenient for the owner and the compliance is rather bad [74].

\subsubsection{Ointments and gels}

Polymeric gels or ointments have been widely used to increase the viscosity and consequently the retention time of eye drops on the ocular surface in order to increase intraocular drug levels $[75,76]$. The most widely used polymers include cellulosic derivatives, poly(vinyl alcohol), sodium hyaluronate, and carbomer. Polymeric gels are classified into two distinct groups: preformed and in situ forming gels. Possible discomfort of an ointment or gel is caused by blurred vision after installation of the ointment or gel $[2,75,77]$.

\subsubsection{Mucoadhesive formulations}

This approach relies on vehicles containing polymers that adhere via non-covalent bonds to conjunctival mucin, thus ensuring contact of the medication with the precorneal tissues until mucin turnover causes elimination of the polymer. This extends the contact time of the drug with the biological tissues and thereby improve the ocular bioavailability [11]. Mucoadhesive polymers are usually hydrocolloids with numerous hydrophilic functional groups such as carboxyl, hydroxyl, amide and sulphate. These groups can establish electrostatic interactions, hydrophobic interactions, van der Waals intermolecular interactions and hydrogen bonding with mucus substrates.

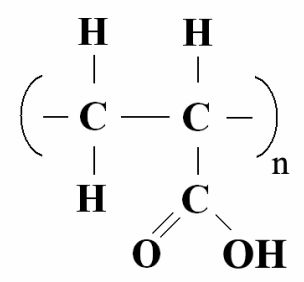

Figure 1.4. General chemical structure of poly(acrylic acid).

Figure 1.4 shows the chemical structure of poly(acrylic acid). The widely used Carbopol ${ }^{\circledR}$ consists polymers of poly(acrylic acid) cross-linked with polyalkenyl ethers or divinyl glycol. 
Other examples of mucoadhesive polymers are hydroxypropylcellulose, polyethylene glycols, dextrans, and hyaluronic acid [5].

\subsubsection{Polymer colloidal systems (nanoparticles and microparticles)}

Polymeric colloidal systems range in size from $10 \mathrm{~nm}$ to micrometers. The drug is dissolved, entrapped, encapsulated or adsorbed in the nano- or microparticle. The binding of the drug depends on the physicochemical properties of the drugs as well as the nano- or microparticle polymer. After optimal drug binding to these particles, the drug absorption in the eye is enhanced significantly in comparison to eye drop solutions. This is attributed to the much slower elimination rates of the particles [76].

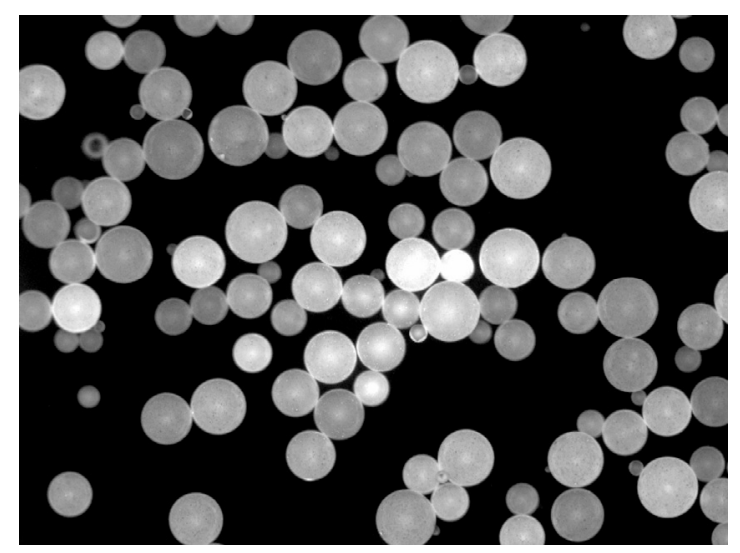

Figure 1.5. An example of poly(methyl methacrylate)-microspheres.

Surface-modified particulate carriers may be used to accommodate a wide variety of drugs. The major development issues in the case of particulates include formulation stability, particle size uniformity, control of drug release rate, and large-scale manufacture of sterile preparation [70]. Examples of polymers used for particulates are poly(hydroxyethyl methacrylate), poly(methyl methacrylate) or chitosan. Figure 1.5 shows a light microscope photograph of poly(methyl methacrylate) microspheres. The average size of the spheres is $240 \mu \mathrm{m}$ in diameter.

\subsubsection{Contact lenses}

Hard contact lenses, soft contact lenses, and intraocular lenses are made from the methacrylate family of polymers. Hard contact lenses are made of poly(methyl methacrylate) (polyMMA). PolyMMA is a hydrophobic, linear chain polymer that is transparent, amorphous, and glassy at 
room temperature. The widely used material for soft contact lenses is poly(hydroxyethyl methacrylate) (polyHEMA).

For soft contact lenses, the methyl ester group in methyl methacrylate is substituted with a hydroxyethyl group. This results in a very hydrophilic polymer, which can slightly be crosslinked with ethylene glycol dimethacrylate (EGDMA) to retain dimensional stability for its use as a lens [78]. Figure 1.6 shows a photograph of a soft contact lens [79].

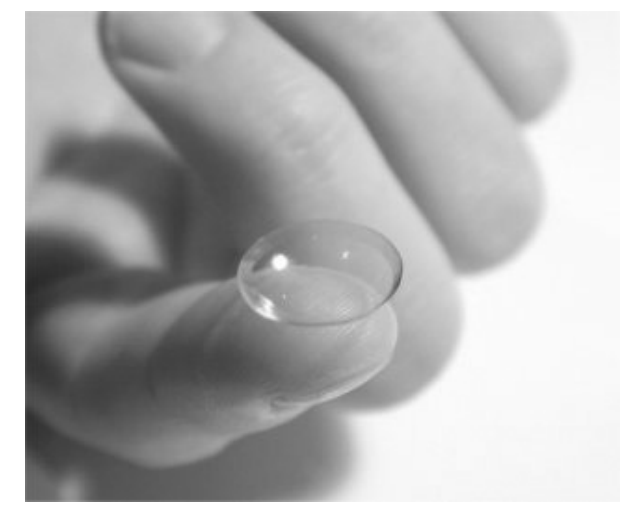

Figure 1.6. A photograph of a soft contact lens [79].

Contact lenses can absorb water-soluble drugs when soaked in drug solutions. Another approach for the delivery of drugs is the encapsulation of ophthalmic drugs in nanoparticles and dispersion of these drug-laden particles in the lens material $[76,80]$.

Although soft contact lenses have excellent comfortable feeling and biocompatibility, the drugloading capacity of a conventional lens is quite low and, as a consequence, it is difficult to achieve therapeutic concentrations in the eye [81]. As mentioned before, the use of soft contact lenses has been associated with the potential risk of developing microbial or fungal keratitis $[50,51]$. The risk of microbial keratitis among contact lens wearers is about 80 -fold greater than among healthy non-wearers. Common organisms associated in these infections are bacteria, such as Pseudomonas aeruginosa and Staphylococci aureus [82].

\subsubsection{Ocular inserts}

An ocular insert provides more controlled, sustained and continuous drug delivery by maintaining an effective drug concentration in the target tissues and yet minimizing the number of applications [76]. There are different kinds of inserts: soluble, biodegradable, nonbiodegradable and hydrogel inserts.

Hydrogels are three-dimensional, hydrophilic, polymer networks capable of imbibing large amounts of water or biological fluids [83]. They have been of great interest to biomaterial 
scientists for many years. Hydrogels may absorb from 10-20\% (an arbitrary lower limit) up to thousands of times their dry weight in water and resemble natural living tissue more than any other class of biomaterials. This is due to their high water content and soft consistency, which is similar to natural tissue. Furthermore, the high water content of the materials contributes to their biocompatibility [83].

There are two different types of gels. When the networks are held together by molecular entanglements, and/or secondary forces including ionic, hydrogen-bonding or hydrophobic forces, the hydrogels are called reversible or physical gels. Hydrogels are called permanent or chemical gels when they are covalently cross-linked networks. In the cross-linked state they reach an equilibrium swelling level in aqueous solutions that depends mainly on the cross-link density [78].

Advantages of ocular inserts are an increased ocular residence, slow constant drug release, accurate dosing, reduction of systemic absorption, better patient compliance, possibility of targeting internal tissues, increased shelf-life, exclusion of preservatives and the possibility of incorporating novel approaches like mucoadhesives, prodrugs or microparticles. Possible disadvantages can be foreign body sensation, movement in the eye, interference with vision, difficult placement (and removal when necessary) and price [84].

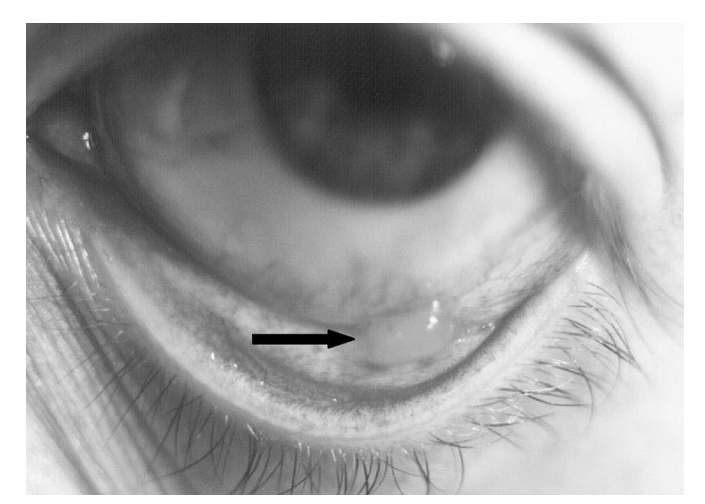

Figure 1.7. A mini-tablet inserted in the conjunctival fornix [85].

As mentioned before, considerable amount of effort has been made in ophthalmic drug delivery since the 1970s [67]. This includes injection therapies, microspheres and polymer carriers. Examples in ocular drug delivery inserts are a mucoadhesive mini-tablet (see Figure 1.7 [85]), a calcium-alginate insert with hydroxyethyl cellulose [86] and chitosan nanoparticles [87]. More recently developed ocular inserts are:

- Gelrite ${ }^{\mathbb{B}}$-based in situ gelling formulations. The concentration of the polymer Gelrite ${ }^{\circledR}$ has a major influence on the drug release as observed by the increase in the amount of drug 
release with an increased polymer concentration from 0.4 to $0.6 \%$. Initially rapid release was observed, gradually approaching constant values for the rest of the time (up to 12 hours in vitro) [88]. These gelling formulations have been developed for many drugs such as timolol maleate [89], ciprofloxacin hydrochloride [90], and indomethacin [91].

- Mydriasert $^{\circledR}$, developed to obtain preoperative mydriasis. Mydriasert ${ }^{\circledR}$ is defined as an insoluble mini-matrix coated with a semi-permeable membrane that increases the local tolerance and regularises the release rate of the active ingredients it contains [92]. It contains two mydriatic drugs (phenylephrine and tropicamide) at a dose corresponding to the content of one eye drop. The time required for mydriasis is slightly longer compared to conventional eye drop treatment [93, 94]. This insert is currently being evaluated in clinical trials in France.

Some attempts developed into a commercially available delivery system. An example is Ocusert $^{\circledR}$, the first rate-controlled drug delivery system developed by Alza Corporation, and first marketed in the USA in 1974 [11, 84]. Ocusert ${ }^{\circledR}$ is an elliptical shaped membrane, which is soft and flexible. It contains the drug pilocarpine, used for the treatment of glaucoma. Alza Corporation developed two inserts, Ocusert Pilo-20 and Ocusert Pilo-40, containing different amounts of drug. Some problems that were observed with these kinds of inserts were foreign body sensation, the disappearance of the insert without noticing by the patient, and disintegration or leakage of the insert [84].

\subsubsection{Our approach}

The use of an ocular device, which releases its drug gradually over a period of time, would be a solution for the delivery of drugs to the eye in both human and veterinary ophthalmology. We developed a novel ocular drug delivery device, called the OphthaCoil. It can be placed in the lower conjunctival fornix, where it releases its drug. The device consists of a metal coil, coated with a hydrogel. A drug is entrapped in the hydrogel coating. Special care is taken on the shape of the device, to avoid spontaneous disappearance, as well as on the integrity of the insert. The device is non-biodegradable to prevent disintegration of the device or leakage of the drug. Consequently, the device must be removed after the drug is released. The interior of the coil can be used as extra reservoir for drugs, to obtain a higher and extended drug release into the tear fluid. This thesis describes the preparation of the device, various methods of using the interior of the coil, and in vitro and in vivo experiments using several drugs. 


\subsection{Outline of this thesis}

Chapter 2 describes the pathway towards our new ocular drug delivery device. It covers the preparation of the device, as well as the first in vitro release experiments with a dye. A first approach to optimize the drug release profile was done using coated metallic filaments in the interior of the coil. Also, a preliminary experiment with two volunteers and devices loaded with a pupil-widening agent was described.

Next, in vivo experiments with Beagle dogs were done in Chapter 3, where we focused on the tolerance of the device in the canine eye. Also preliminary drug release of the antibiotic pradofloxacin, developed by Bayer HealthCare AG (Monheim, Germany), was described. The inner cavity of the device was filled with the filaments, as introduced in Chapter 2.

Chapter 4 describes a clinical trial using the device with the metallic filaments in the inner cavity to investigate the tolerance and ease of the insertion/removal procedure in the human eye. Here, five volunteers received the insert in the eye for a 2-hour period. The device did not contain a drug. Ophthalmologic examinations, including photographs, as well as questionnaires for the volunteers, were performed to evaluate the device.

A second method of increasing the drug capacity of the device by using drug-loaded polymeric microparticles was described in Chapter 5. Here, microparticles consisting of poly(HEMA) were prepared by suspension polymerization. The particles were loaded with a dye or an antibiotic, introduced into the inner cavity of the coil, and the release profiles in vitro were investigated in a flow system. Also, the flexibility of the coil was investigated.

Chapter 6 shows a third approach to increase the drug capacity of the device and to optimize the release profile. Here, a drug-loaded polymeric rod was made, which was placed in the inner cavity of the coil. The release of the antibiotic pradofloxacin in the in vitro flow system was measured. Furthermore, Beagle dogs received an insert under the eyelid for 72 hours and tear fluid samples were collected to measure the concentration of the antibiotic in the eye.

In Chapter 7, a new method of filling the inner cavity of the coil was compared to the method described in Chapter 6. Here, a textile fiber was soaked into a highly concentrated drug solution and the dried fiber was inserted double in the inner cavity of the coil. An in vitro experiment compared the release of the drugs chloramphenicol and cyclosporine A according to both approaches: the fiber and the polymer rod. Furthermore, we describe in vivo experiments with Beagle dogs, where we inserted a device with two pupil-widening agents in the eye and observed the pupil dilation and pupil response to light over time. Finally, coils were made with larger diameters and lengths. These coils were inserted into the eyes of two 
horses. The tolerance and persistence of the device was evaluated over a time period of 24 hours.

Chapter 8 describes the possibility of using a porous substrate based on ultra high molecular weight polyethylene (UHMWPE), called Solupor ${ }^{\circledR}$ (DSM Solutech, Geleen, the Netherlands), as a matrix for the controlled delivery of drugs. The substrate is a thin, porous film, which can be placed in the conjunctival fornix as well. The substrate can be loaded using different methods. The matrix can be impregnated with a drug via precipitation of the drug in the pores of the substrate, a drug-containing hydrogel coating layer prepared by photopolymerization, or a combination of both methods can be used. The release of the antibiotic chloramphenicol from the substrate is also described in this chapter.

Finally, a general discussion will be given in Chapter 9. Here, the different methods of increasing the capacity of the OphthaCoil will be discussed, as well as the advantages and possible disadvantages of the insert. Also, possible applications of both the OphthaCoil and the porous substrate will be discussed.

\section{References}

1. Smith T.E. Molecular cell biology. In: Textbook of Biochemistry with clinical correlations. Edited by Devlin T.M., 4th edn. New York, Wiley-Liss, Inc (1997) 919-979.

2. Davies N.M. Biopharmaceutical considerations in topical ocular drug delivery. Clin Exp Pharmacol Physiol 27 (7) (2000) 558-562.

3. Kaur I.P. and Kanwar M. Ocular preparations: the formulation approach. Drug Dev Ind Pharm 28 (5) (2002) 473-493.

4. Prausnitz M.R. and Noonan J.S. Permeability of cornea, sclera, and conjunctiva: a literature analysis for drug delivery to the eye. J Pharm Sci 87 (12) (1998) 1479-1488.

5. Saettone M.F. Progress and problems in ophthalmic drug delivery. Business briefing: Pharmatech (2002) 1-6.

6. Bourges J.L., Bloquel C., Thomas A., Froussart F., Bochot A., Azan F., Gurny R., BenEzra D. and Behar-Cohen F. Intraocular implants for extended drug delivery: therapeutic applications. Adv Drug Deliv Rev 58 (11) (2006) 1182-1202.

7. Hosoya K., Lee V.H. and Kim K.J. Roles of the conjunctiva in ocular drug delivery: a review of conjunctival transport mechanisms and their regulation. Eur J Pharm Biopharm 60 (2) (2005) 227240.

8. Urtti A. Challenges and obstacles of ocular pharmacokinetics and drug delivery. Adv Drug Deliv Rev 58 (11) (2006) 1131-1135.

9. Mannermaa E., Vellonen K.S. and Urtti A. Drug transport in corneal epithelium and blood-retina barrier: emerging role of transporters in ocular pharmacokinetics. Adv Drug Deliv Rev 58 (11) (2006) 1136-1163.

10. Lang J.C. Ocular drug delivery conventional ocular formulations. Adv Drug Deliv Rev 16 (1995) 39-43.

11. Ali Y. and Lehmussaari K. Industrial perspective in ocular drug delivery. Adv Drug Deliv Rev 58 (11) (2006) 1258-1268. 
12. Hamalainen K.M., Kananen K., Auriola S., Kontturi K. and Urtti A. Characterization of paracellular and aqueous penetration routes in cornea, conjunctiva, and sclera. Invest Ophthalmol Vis Sci 38 (3) (1997) 627-634.

13. Shargel L., Wu-Pong S. and Yu A.B. Applied Biopharmaceutics \& Pharmacokinetics, 5th edn. New York: McGray-Hill; 2005.

14. Schoenwald R.D. Chapter 9: Ocular pharmacokinetics. In: Textbook of ocular pharmacology. Edited by Zimmermann T.J. Philadelphia, Lippinocott-Raven Publishers (1997) 119-138.

15. Bodor N. and Buchwald P. Ophthalmic drug design based on the metabolic activity of the eye: soft drugs and chemical delivery systems. AAPS J 7 (4) (2005) E820-833.

16. Drinkwater M. Stand by for implantable contact lenses. http://www.onset.unsw.edu.au/ (2007).

17. Middleton D.L., Leung S.S. and Robinson J.R. Ocular bioadhesive drug delivery systems. In: Bioadhesive drug delivery systems. Edited by Lenaerts V. and Gurny R. Boca Raton, Florida, CRC Press, Inc. (1990) 179-202.

18. Dartt D.A. Regulation of mucin and fluid secretion by conjunctival epithelial cells. Prog Retin Eye Res 21 (6) (2002) 555-576.

19. Baeyens V. and Gurny R. Chemical and physical parameters of tears relevant for the design of ocular drug delivery formulations. Pharm Acta Helv 72 (4) (1997) 191-202.

20. Ghate D. and Edelhauser H.F. Ocular drug delivery. Expert Opin Drug Deliv 3 (2) (2006) 275-287.

21. Asbell P.A., Dualan I., Mindel J., Brocks D., Ahmad M. and Epstein S. Age-related cataract. Lancet 365 (9459) (2005) 599-609.

22. Boros J., Newitt P., Wang Q., McAvoy J.W. and Lovicu F.J. Sef and Sprouty expression in the developing ocular lens: implications for regulating lens cell proliferation and differentiation. Semin Cell Dev Biol 17 (6) (2006) 741-752.

23. Beebe D.C. Chapter 5: The Lens. In: Adler's physiology of the eye: clinical application. Edited by Kaufman P.L. and Alm A., 10th edn. St. Louis, Missouri, Mosby, Inc. (2003) 117-158.

24. Geroski D.H. and Edelhauser H.F. Drug delivery for posterior segment eye disease. Invest Ophthalmol Vis Sci 41 (5) (2000) 961-964.

25. Pardue M.T., Hejny C., Gilbert J.A., Phillips M.J., Geroski D.H. and Edelhauser H.F. Retinal function after subconjunctival injection of carboplatin in fibrin sealant. Retina 24 (5) (2004) 776-782.

26. Edelhauser H.F. and Ubels J.L. Chapter 4: Cornea and Sclera. In: Adler's physiology of the eye. Edited by Kaufman P.L. and Alm A., 10th edn. St. Louis, Mosby Inc. (2003) 47-114.

27. Watson P.G. and Young R.D. Scleral structure, organisation and disease. A review. Exp Eye Res 78 (3) (2004) 609-623.

28. Gabelt B.T. and Kaufman P.L. Aqueous humor hydrodynamics. In: Adler's physiology of the eye. Edited by Kaufman P.L. and Alm A. St. Louis, Mosby Inc. (2003) 237-289.

29. Lund-Andersen H. and Sander B. Chapter 9: The vitreous. In: Adler's physiology of the eye. Edited by Kaufman P.L. and Alm A. St. Louis, Mosby Inc. (2003) 293-316.

30. National-Eye-Institute. Vision loss from eye disease will increase as Americans age. NEI press release (2004).

31. Sharma R.K. and Ehinger B.E.J. Development and structure of the retina. In: Adler's physiology of the eye. Edited by Kaufman P.L. and Alm A., 10th edn. St. Louis, Mosby Inc. (2003) 319-347.

32. Kardon R. The pupil. In: Adler's physiology of the eye. Edited by Kaufman P.L. and Alm A. St. Louis, Mosby Inc. (2003).

33. Vision 2020: the Right to Sight. http://www.v2020.org/ (2007).

34. Congdon N.G., Friedman D.S. and Lietman T. Important causes of visual impairment in the world today. JAMA 290 (15) (2003) 2057-2060.

35. Clark A.F. and Yorio T. Ophthalmic drug discovery. Nat Rev Drug Discov 2 (6) (2003) 448-459.

36. Nijkamp M.D., Dolders M.G., de Brabander J., van den Borne B., Hendrikse F. and Nuijts R.M. Effectiveness of multifocal intraocular lenses to correct presbyopia after cataract surgery: a randomized controlled trial. Ophthalmology 111 (10) (2004) 1832-1839.

37. Bloemendal H., de Jong W., Jaenicke R., Lubsen N.H., Slingsby C. and Tardieu A. Ageing and vision: structure, stability and function of lens crystallins. Prog Biophys Mol Biol 86 (3) (2004) 407485 . 
38. Mouly S., Mahe I., Haouchine B., Sanson-le-Pors M.J., Blain P., Tillet Y., Dewailly J., Mongold J.J. and Bergmann J.F. Pharmacodynamics of a new ophthalmic mydriatic insert in healthy volunteers: potential alternative as drug delivery system prior to cataract surgery. Basic Clin Pharmacol Toxicol 98 (6) (2006) 547-554.

39. Taban M., Behrens A., Newcomb R.L., Nobe M.Y., Saedi G., Sweet P.M. and McDonnell P.J. Acute endophthalmitis following cataract surgery: a systematic review of the literature. Arch Ophthalmol 123 (5) (2005) 613-620.

40. Ziekenhuisstatistieken. http://www.prismant.nl (2007).

41. Ambati J., Ambati B.K., Yoo S.H., Ianchulev S. and Adamis A.P. Age-related macular degeneration: etiology, pathogenesis, and therapeutic strategies. Surv Ophthalmol 48 (3) (2003) 257-293.

42. Hughes P.M., Olejnik O., Chang-Lin J.E. and Wilson C.G. Topical and systemic drug delivery to the posterior segments. Adv Drug Deliv Rev 57 (14) (2005) 2010-2032.

43. Barouch F.C. and Gragoudas E.S. Progress in age-related macular degeneration: a review. Adv Stud Ophthalmol 3 (3) (2006) 60-66.

44. Wong T. and Mitchell P. The eye in hypertension. Lancet 369 (9559) (2007) 425-435.

45. Fechtner R.D. and Kooner K.S. Definitions and classification of glaucoma. In: Textbook of Ocular Pharmacology. Edited by Zimmermann T.J., et al. Philadelphia, Lippincott-Raven Publishers (1997).

46. McCluskey P. and Powell R.J. The eye in systemic inflammatory diseases. Lancet 364 (9451) (2004) 2125-2133.

47. Kasi P.M., Gilani A.I., Ahmad K. and Janjua N.Z. Blinding trachoma: a disease of poverty. PLoS Med 1 (2) (2004) e44.

48. Weir E., Haider S. and Telio D. Trachoma: leading cause of infectious blindness. CMAJ 170 (8) (2004) 1225.

49. Dart J.K., Stapleton F. and Minassian D. Contact lenses and other risk factors in microbial keratitis. Lancet 338 (8768) (1991) 650-653.

50. Chang D.C., Grant G.B., O'Donnell K., Wannemuehler K.A., Noble-Wang J., Rao C.Y., Jacobson L.M., Crowell C.S., Sneed R.S., Lewis F.M. et al Multistate outbreak of Fusarium keratitis associated with use of a contact lens solution. JAMA 296 (8) (2006) 953-963.

51. Iyer S.A., Tuli S.S. and Wagoner R.C. Fungal keratitis: emerging trends and treatment outcomes. Eye Contact Lens 32 (6) (2006) 267-271.

52. Williams R., Airey M., Baxter H., Forrester J., Kennedy-Martin T. and Girach A. Epidemiology of diabetic retinopathy and macular oedema: a systematic review. Eye 18 (10) (2004) 963-983.

53. Rudnick D.E., Noonan J.S., Geroski D.H., Prausnitz M.R. and Edelhauser H.F. The effect of intraocular pressure on human and rabbit scleral permeability. Invest Ophthalmol Vis Sci 40 (12) (1999) 3054-3058.

54. Ozturk F., Kurt E., Inan U.U., Kortunay S., Ilker S.S., Basci N.E. and Bozkurt A. The effects of prolonged acute use and inflammation on the ocular penetration of topical ciprofloxacin. Int J Pharm 204 (1-2) (2000) 97-100.

55. Essex R.W., Yi Q., Charles P.G. and Allen P.J. Post-traumatic andophthalmitis. Ophthalmology 111 (11) (2004) 2015-2022.

56. Yokoi N. and Komuro A. Non-invasive methods of assessing the tear film. Exp Eye Res 78 (3) (2004) 399-407.

57. Bielory L. Ocular allergy and dry eye syndrome. Curr Opin Allergy Clin Immunol 4 (5) (2004) 421424.

58. Gobbels M. and Spitznas M. Corneal epithelial permeability of dry eyes before and after treatment with artificial tears. Ophthalmology 99 (6) (1992) 873-878.

59. Brewitt H. and Sistani F. Dry eye disease: the scale of the problem. Surv Ophthalmol 45 (2) (2001) S199-202.

60. Hornof M., Toropainen E. and Urtti A. Cell culture models of the ocular barriers. Eur J Pharm Biopharm 60 (2) (2005) 207-225.

61. Hamalainen K.M., Kontturi K., Auriola S., Murtomaki L. and Urtti A. Estimation of pore size and pore density of biomembranes from permeability measurements of polyethylene glycols using an effusion-like approach. J Control Release 49 (2-3) (1997) 97-104. 
62. Jarvinen K., Jarvinen T. and Urtti A. Ocular absorption following topical delivery. Adv Drug Deliv Rev 16 (1) (1995) 3-19.

63. Watsky M.A., Jablonski M.M. and Edelhauser H.F. Comparison of conjunctival and corneal surface areas in rabbit and human. Curr Eye Res 7 (5) (1988) 483-486.

64. Horibe Y., Hosoya K., Kim K.J., Ogiso T. and Lee V.H. Polar solute transport across the pigmented rabbit conjunctiva: size dependence and the influence of 8-bromo cyclic adenosine monophosphate. Pharm Res 14 (9) (1997) 1246-1251.

65. Cruysberg L.P., Nuijts R.M., Geroski D.H., Koole L.H., Hendrikse F. and Edelhauser H.F. In vitro human scleral permeability of fluorescein, dexamethasone-fluorescein, methotrexate-fluorescein and rhodamine $6 \mathrm{G}$ and the use of a coated coil as a new drug delivery system. J Ocul Pharmacol Ther 18 (6) (2002) 559-569.

66. Kao J.C., Geroski D.H. and Edelhauser H.F. Transscleral permeability of fluorescent-labeled antibiotics. J Ocul Pharmacol Ther 21 (1) (2005) 1-10.

67. Ding S. Recent developments in ophthalmic drug delivery. Pharm Sci Technol Today 1 (8) (1998) 328-335.

68. Lv F.F., Li N., Zheng L.Q. and Tung C.H. Studies on the stability of the chloramphenicol in the microemulsion free of alcohols. Eur J Pharm Biopharm 62 (3) (2006) 288-294.

69. Lee S.B., Geroski D.H., Prausnitz M.R. and Edelhauser H.F. Drug delivery through the sclera: effects of thickness, hydration, and sustained release systems. Exp Eye Res 78 (3) (2004) 599-607.

70. Barbu E., Verestiuc L., Nevell T.G. and Tsibouklis J. Polymeric materials for ophthalmic drug delivery: trends and perspectives. J Mater Chem 16 (34) (2006) 3439-3443.

71. Baudouin C. Allergic reaction to topical eyedrops. Curr Opin Allergy Clin Immunol 5 (5) (2005) 459-463.

72. Tears Naturale. Products for dry eye relief. http://www.tearsnaturale.com/ (2007).

73. Salyani A. and Birt C. Evaluation of an eye drop guide to aid self-administration by patients experienced with topical use of glaucoma medication. Can J Ophthalmol 40 (2) (2005) 170-174.

74. Baeyens V.V., Percicot C., Zignani M., Deshpande A.A., Kaltsatos V.V. and Gurny R. Ocular drug delivery in veterinary medicine. Adv Drug Deliv Rev 28 (3) (1997) 335-361.

75. Baydoun L., Furrer P., Gurny R. and Muller-Goymann C.C. New surface-active polymers for ophthalmic formulations: evaluation of ocular tolerance. Eur J Pharm Biopharm 58 (1) (2004) 169175.

76. Sultana Y., Jain R., Aqil M. and Ali A. Review of ocular drug delivery. Curr Drug Deliv 3 (2) (2006) 207-217.

77. Le Bourlais C., Acar L., Zia H., Sado P.A., Needham T. and Leverge R. Ophthalmic drug delivery systems--recent advances. Prog Retin Eye Res 17 (1) (1998) 33-58.

78. Peppas N.A. Chapter 2.5: Hydrogels. In: Biomaterials Science: an introduction to materials in medicine. Edited by Ratner B.D., Hoffman A.S., Schoen F.J. and Lemons J.E., 2nd edn. San Diego, California, Elsevier Academic Press (2004).

79. Trends contact lenses. http://www.designer-fashion-trends.com (2007).

80. Gulsen D. and Chauhan A. Ophthalmic drug delivery through contact lenses. Invest Ophthalmol Vis Sci 45 (7) (2004) 2342-2347.

81. Hiratani H., Fujiwara A., Tamiya Y., Mizutani Y. and Alvarez-Lorenzo C. Ocular release of timolol from molecularly imprinted soft contact lenses. Biomaterials 26 (11) (2005) 1293-1298.

82. Alfonso E.C., Miller D., Cantu-Dibildox J., O'Brien T.P. and Schein O.D. Fungal keratitis associated with non-therapeutic soft contact lenses. Am J Ophthalmol 142 (1) (2006) 154-155.

83. Peppas N.A., Bures P., Leobandung W. and Ichikawa H. Hydrogels in pharmaceutical formulations. Eur J Pharm Biopharm 50 (1) (2000) 27-46.

84. Saettone M.F., Salminen, L. Ocular inserts for topical delivery. Adv Drug Deliv Rev 16 (1995) 95106.

85. Ceulemans J., Vermeire A., Adriaens E., Remon J.P. and Ludwig A. Evaluation of a mucoadhesive tablet for ocular use. J Control Release 77 (3) (2001) 333-344. 
86. Fuchs-Koelwel B., Koelwel C., Gopferich A., Gabler B., Wiegrebe E. and Lohmann C.P. Tolerance of a new calcium-alginate-insert for controlled medication therapy of the eye. Ophthalmologe 101 (5) (2004) 496-499.

87. de Campos A.M., Diebold Y., Carvalho E.L., Sanchez A. and Alonso M.J. Chitosan nanoparticles as new ocular drug delivery systems: in vitro stability, in vivo fate, and cellular toxicity. Pharm Res 21 (5) (2004) 803-810.

88. Sultana Y., Aqil M. and Ali A. Ion-activated, Gelrite-based in situ ophthalmic gels of pefloxacin mesylate: comparison with conventional eye drops. Drug Deliv 13 (3) (2006) 215-219.

89. Rozier A., Mazuel C., Grove J. and Plazonnet B. Gelrite ${ }^{\circledR}$ : a novel, ion-activated, in-situ gelling polymer for ophthalmic vehicles. Effect on bioavailability of timolol. Int J Pharm 57 (1989) 163168.

90. Balasubramaniam J. and Pandit J.K. Ion-activated in situ gelling systems for sustained ophthalmic delivery of ciprofloxacin hydrochloride. Drug Deliv 10 (3) (2003) 185-191.

91. Balasubramaniam J., Kant S. and Pandit J.K. In vitro and in vivo evaluation of the Gelrite gellan gum-based ocular delivery system for indomethacin. Acta Pharm 53 (4) (2003) 251-261.

92. Aiache J.M., el Meski S., Serpin G., Beyssac E. and Conti R. Local tolerance and in vivo release study of a new ocular drug delivery system. Drug Deliv Systems and Sciences 1 (3) (2001) 85-87.

93. Korobelnik J.F., Tavera C., Renaud-Rougier M.B., El Meski S. and Colin J. The Mydriasert insert: an alternative to eye drops for preangiographic mydriasis. J Fr Ophtalmol 27 (8) (2004) 897-902.

94. Caruba T., Couffon-Partant C., Oliary J., Tadayoni R., Limelette N. and Gaudric A. Efficacy and efficiency of preoperative mydriasis: drops versus ocular insert. J Fr Ophtalmol 29 (7) (2006) 789795. 


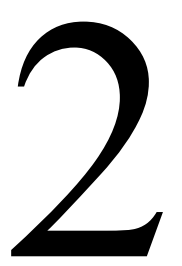

Flexible coils with a drug-releasing hydrophilic coating: A new platform for controlled delivery of drugs to the eye?

Rachel T. Pijls, Hans H.L. Hanssen, Rudy M.M.A. Nuijts and Leo H. Koole Adapted from: Biomedical Materials and Engineering 14 (2004) 383-393 


\section{Abstract}

Delivery of drugs to the front-side of the eye is routinely done via eye drops. It is known that approximately $80 \%$ of each eye-drop is lost, as a result of rapid clearance of the tear fluid via the naso-lacrymal canal. Consequently, repeated administration through several droplets is usually necessary to achieve a desired effect, e.g., mydriasis (widening of the pupil) prior to corneal surgery. Studies with a new ocular drug delivery device are reported. The new device is believed to provide a basis for more convenient and efficient method for ocular drug delivery. The device is a metallic coil with a hydrophilic, drug-containing polymeric coating. The coil is placed in the conjunctival fornix (under the lower eye-lid), and the drug is released slowly, by diffusion into the tear fluid. The capacity of the device could be increased by using the lumen of the coils as a depot for the drug to be released. Preliminary experiments with the new device are reported. These experiments were performed largely in vitro, but partly also in vivo. The latter experiments comprised release of the fluorescent dye, and delivery of atropine (a potent mydriatic agent), in the eyes of several healthy volunteers. The first results obtained with the new device indicate its potential utility. It is discussed that much more research and development work is required, e.g., to define the optimal design of the coil in order to minimise the risk for irritation. Furthermore, the parameters that define the kinetics of the intraocular drug release must be defined and optimized with respect to the exact application. 


\subsection{Introduction}

Biocompatible polymeric coatings continue to attract considerable interest in the biomaterials research field [1-11]. Moreover, various medical devices with a polymeric coating have found their way into clinical practice in the recent years. The modern coronary stent may serve as an illustrative example $[12,13]$. The original stent was, in essence, a mechanical device that serves as a scaffold for the vessel wall after balloon dilatation. The stent was designed to prevent "elastic recoil", i.e. spontaneous re-narrowing of the coronary artery. In many cases, however, the stent proved to be ineffective in preventing so-called in-stent restenosis, which is cellular response to the presence of the stent. Intimal hyperplasia, i.e. extensive proliferation of smooth muscle cells in the media of the blood vessel wall, causes in-stent restenosis. The intimal layer thickens, protrudes through the stent mesh, and impedes the blood flow through the stent. It has been shown in animal models that the use of a polymeric coating on the surface of a stent can inhibit intimal hyperplasia. This inhibitory effect can be increased further through incorporation of a drug in the stent coating. The drug is then slowly released at the stent's surface. The most powerful drugs, in this respect, appear to be antimitotic agents, such as paclitaxel $[13,14]$ and sirolimus (rapamycin) $[13,15]$. The polymer-coated, drug-loaded coronary stent has become a landmark in the development of bioactive coatings for medical devices $[13,16,17]$.

Our work on biocompatible polymeric coatings relates to new hydrophilic biomaterials which are applied onto long metallic wires $[8,18,19]$. The coating materials are copolymers of $\mathrm{N}$ vinyl pyrrolidone (NVP, hydrophilic building block), and butyl methacrylate (BMA, hydrophobic building block) [20,21]; this family of coating materials was given the generic name SlipSkin ${ }^{\circledR}$. The ratio of NVP:BMA determines the hydrophilicity of the coating which, in turn, steers important physical parameters such as degree of swelling and lubricity. To apply the coatings onto metallic wires, we use an extrusion-like application technique, which was described previously [18]. This approach allows accurate control over the thickness of the coating layer in the range $2-10 \mu \mathrm{m}$. Based on this procedure new hydrophilic coiled guide wires were developed. These have a well-defined hydrophilic coating with ideal slipperywhen-wet lubricity characteristics. The coils lead to minimal haemolysis in contact with full blood, they have low thrombogenicity (in vitro and in vivo), and hardly activate contacting blood platelets (in vitro). It has been shown that it is possible to incorporate pharmacologically active agents in SlipSkin ${ }^{\circledR}$ coatings. For example, we have introduced heparin in the coatings, and it could be shown that: (i) heparin "survives" the procedure of application of the coating [19]; (ii) release of heparin occurs readily upon immersion of coated wires or coils in an 
aqueous solution; (iii) not all heparin is released; part of it remains entrapped in the coating; (iv) use of heparin-releasing coils and guide wires may minimize the risk for formation of emboli at the surface of the coils (contact activation of blood coagulation is inhibited); (v) release of heparin occurs fast, e.g. within $30 \mathrm{~min}$, after immersion in the circulation, which is acceptable for short interventional procedures (PTCA), but not suitable for long-term release $[8,19]$.

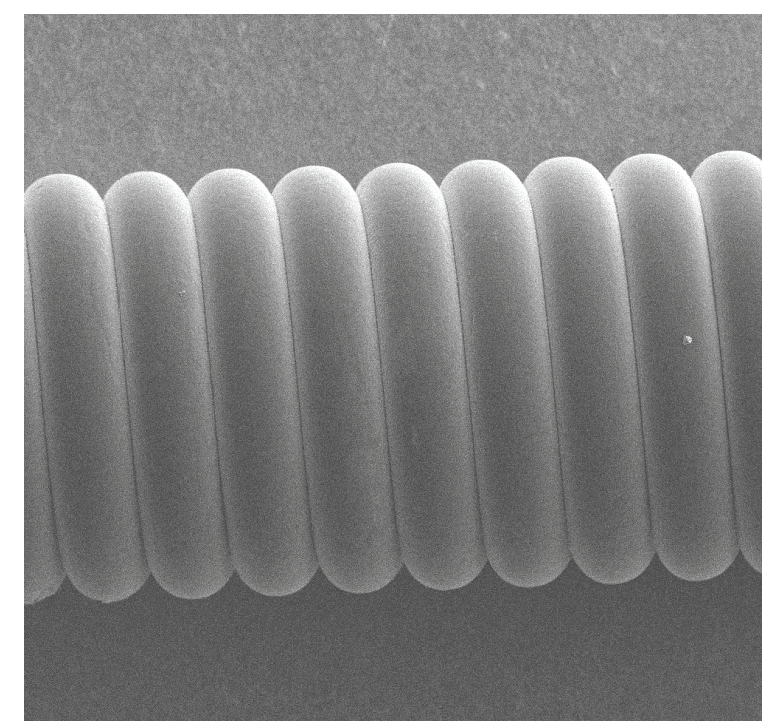

Figure 2.1. Scanning electron micrograph showing a mid-section of a Slipskin ${ }^{\circledR}$-coated coil; the diameter of the coil is $600 \mu \mathrm{m}$; the diameter of the coated wire is $87 \mu \mathrm{m}$.

Herein, we wish to report on a new application of metallic coils with a drug-containing SlipSkin ${ }^{\circledR}$ coating. We focus on short coils (length $16 \mathrm{~mm}$, diameter appr. $450 \mu \mathrm{m}$ ); a typical example is shown in Figure 2.1. At the onset of this study, our idea was to investigate whether a coil like this can be laid in the lower conjunctival fornix of the eye (i.e. under the lower eyelid) without causing irritation to the patient. If this is the case, then the coil could be used as a temporary vehicle for local release of a drug to the tear fluid of the eye. The coil can then potentially serve as an alternative to the administration of drugs to the eye via eye drops. Obviously, the coil will start to absorb water from the tear fluid after its introduction in the fornix, and concomitant drug release will occur.

The device has two different depots for the drug that is to be released: (i) the hydrophilic coating on the coil, and (ii) the lumen of the coils, which is a relatively big volume. This approach is attractive for at least 2 reasons: 
- The amount of the drug that is administered to the eye can be controlled more accurately, as compared to eye drops;

- Drug release over a time-span can be realized (e.g. several days), provided that the release kinetics can be controlled.

We report here preliminary studies of: (i) use of a short spiral, charged with the dye fluorescein, in the eye of a healthy volunteer; (ii) use of a short spiral for the release of atropine, a well-known mydriatic (pupil-widening) agent [22], also in the eye of a healthy volunteer; (iii) possibilities to utilize the coils and their inner lumen for the prolonged release of the antibiotic agent chloramphenicol [23]. The chemical structures of the agents are given in Figure 2.2. Based on these preliminary findings, the potential utility of this device for ocular drug delivery is discussed briefly.

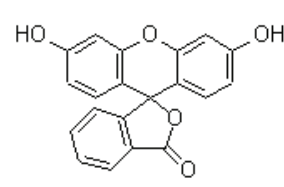

Fluorescein

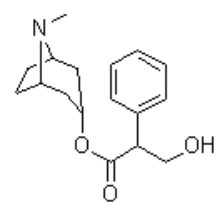

Atropine

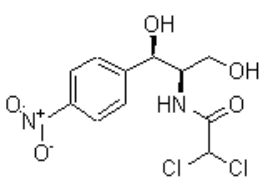

Chloramphenicol

Figure 2.2. The chemical structures of fluorescein, atropine and chloramphenicol.

\subsection{Materials and methods}

Four variations of the SlipSkin ${ }^{\circledR}$-copolymer were prepared on a $100 \mathrm{~g}$ scale; the ratios of the hydrophilic building block (NVP) : hydrophobic building block (BMA) were as compiled in Table 2.1. The materials were prepared in free-radical bulk polymerization reactions, using $\alpha, \alpha^{\prime}$-azoisobutyronitrile (AIBN) as the polymerization initiator. The procedure afforded the desired copolymers as hard opaque rods.

Table 2.1. The composition of the different coating biomaterials used in this work.

\begin{tabular}{ccc} 
Materials & Mol\% of NVP & Mol\% of BMA \\
\hline $90-10$ & 90 & 10 \\
$80-20$ & 80 & 20 \\
$70-30$ & 70 & 30 \\
$60-40$ & 60 & 40 \\
\hline
\end{tabular}


The rods were cut into pieces of approximately $1 \mathrm{~g}$. These were dissolved in N-methyl pyrrolidone (NMP) by mechanical stirring overnight; the concentration was set at a copolymer : solvent ratio of 10 : 90 (mass : mass). This resulted in highly viscous but homogeneous solutions. Each copolymer solution was split into four portions: three portions of $200 \mathrm{~g}$, and the remainder of appr. $400 \mathrm{~g}$. The large portion served as backup and was stored at $4^{\circ} \mathrm{C}$. Additives were dissolved as outlined in Table 2.2. Clear solutions were obtained readily, by mechanical stirring at room temperature. For the atropine-containing solutions, gentle heating was required for complete homogeneity.

Table 2.2. The composition of the different drug-loaded coatings.

\begin{tabular}{ccccc}
\hline $\begin{array}{c}\text { Solution } \\
(200 \mathrm{~mL})\end{array}$ & Additive & $\begin{array}{c}\text { Amount of } \\
\text { copolymer }(\mathrm{g})\end{array}$ & $\begin{array}{c}\text { Amount of } \\
\text { additive }(\mathrm{mg})\end{array}$ & Code \\
\hline \multirow{2}{*}{ Slipskin $^{\circledR}$ 90-10 } & Fluorescein & 20 & 200 & $90-10-\mathrm{F}$ \\
& Atropine & 20 & 3000 & $90-10-\mathrm{A}$ \\
\hline \multirow{2}{*}{ Slipskin $^{\circledR} 80-20$} & Fluorescein & 20 & 200 & $80-20-\mathrm{F}$ \\
& Atropine & 20 & 3000 & $80-20-\mathrm{A}$ \\
\hline \multirow{3}{*}{ Slipskin $^{\circledR} 70-30$} & Fluorescein & 20 & 200 & $70-30-\mathrm{F}$ \\
& Atropine & 20 & 3000 & $70-30-\mathrm{A}$ \\
& Chloramphenicol & 13.3 & 13300 & $70-30-\mathrm{C}$ \\
\hline \multirow{2}{*}{ Slipskin $^{\circledR} 60-40$} & Fluorescein & 20 & 200 & $60-40-\mathrm{F}$ \\
& Atropine & 20 & 3000 & $60-40-\mathrm{A}$ \\
\hline
\end{tabular}

The resulting solutions were used in our extrusion-like coating procedure, which was described before. It may be worthwhile to repeat here that the procedure is surprisingly quick and precise: thousands of meters of wire can be coated with a polymer layer of uniform thickness in the range $2-10 \mu \mathrm{m}$; the maximum variation of the thickness is $<10 \%$. The normal speed is approximately 2000 meters per hour.

The present experiments were started with a commercially available stainless steel wire with a diameter of $76 \mu \mathrm{m}$. The wire was first coated with a thin layer of a binding polymer (poly(ethersulfone), thickness $1 \mu \mathrm{m}$ ); the length was appr. 2000 meters. Parts (appr. 200 meters each) were then coated with two layers of $90-10-\mathrm{F}, 80-20-\mathrm{F}, 70-30-\mathrm{F}$, or $60-40-\mathrm{F}$; the hydrophilic dye-containing layer had a thickness of approximately $2.5 \mu \mathrm{m}$. Other parts (also appr. 200 meters each) were coated with two layers of 90-10-A, 80-20-A, 70-30-A, and 60-40A; the thickness of the hydrophilic atropine-containing coating was appr. $6.0 \mu \mathrm{m}$. The remainder of the wire with the binding polymer coating was coated with 70-30-C, partly with 
two layers (thickness of the hydrophilic layer appr. $2 \mu \mathrm{m}$ ), and partly with four layers (thickness of the hydrophilic layer appr. $5 \mu \mathrm{m}$ ). Wires were coiled around a core wire with a diameter of $300 \mu \mathrm{m}$. The inner core wire was later removed so that a flexible coil was obtained. A typical scanning electron micrograph of these coils is shown in Figure 2.1.

\subsubsection{Fluorescein release experiments}

The coils with the coatings $90-10-\mathrm{F}, 80-20-\mathrm{F}, 70-30-\mathrm{F}$ and $60-40-\mathrm{F}$ were cut into pieces of 3 $\mathrm{cm}$. These were placed in Eppendorf vials, filled with $1500 \mu \mathrm{L}$ of Tris buffer solution. The coils were removed after $0.5,1,2,3,5,10,15,30,60$ and 90 minutes, and after 2, 3, 4, 6, 8, 12, 14 and 17 hours. All experiments were done in triplo. After removal of each coil, the contents of the Eppendorf vial were homogenized and stored at $4{ }^{\circ} \mathrm{C}$. The fluorescein concentrations were then measured on an automated spectrophotometer, using a 96-wells plate. For calibration, some of the wells were filled with a series of fluorescein solutions of known concentration. Several smaller pieces (appr. $16 \mathrm{~mm}$ ) were cut from the coil with the 80-20-F coating. These were inspected for the absence of sharp edges, and subsequently placed by an experienced ophthalmologist under the lower eyelid of a healthy volunteer. Release of the dye and its distribution over the tear film was photographed with a fluorescence-sensitive camera. The coil was removed from the eye after appr. $1 \mathrm{~h}$.

\subsubsection{Atropine release experiments}

The coil with the coating 90-10-A was cut into pieces with a length between 1.55 and $1.70 \mathrm{~mm}$. The ends were carefully inspected for the absence of any sharp edges. Two healthy human volunteers were recruited. Volunteer 1 received a $1.67-\mathrm{mm}$ long coil in the right eye, and volunteer 2 received a $1.55-\mathrm{mm}$ long coil in the right eye. Mydriasis (pupil-widening) was evaluated through photography. The coils were removed from the eye after appr. $2 \mathrm{~h}$.

\subsubsection{Chloramphenicol release experiments}

These experiments were done in vitro, using the standard zone of inhibition assay. Coils with the coating 70-30-C (2 layers, and three short chloramphenicol-loaded wires (vide infra) in the lumen) were placed onto bacterial cultures (E. coli), and the release of the antibiotic was evaluated on the basis of the bacterium-free zone around the coil, after $24 \mathrm{~h}$. To increase the antibiotic loading of the coils, we introduced 1,2, or 3 straight pieces of the wire 70-30-C (four layers) into the lumen of the coil (vide infra). 


\subsection{Results}

\subsubsection{Swelling of the hydrophilic coating materials}

Cylindrical buttons with a diameter of $10 \mathrm{~mm}$ and a height of $4 \mathrm{~mm}$, machined from the rods of the materials Slipskin ${ }^{\circledR} 90-10,80-20,70-30$ and $60-40$ as obtained directly after the polymer synthesis, were immersed in Tris buffer at ambient temperature (3 buttons for each material). Swelling of all materials was complete after 1 week. Figure 2.3 shows the samples in the dry state, as well as in the swollen state. The mass of the SlipSkin ${ }^{\circledR} 90-10$ buttons increased by approximately $250 \%$ upon the uptake of water. For the other materials (SlipSkin ${ }^{\circledR} 80-20,70-30$ and 60-40), the masses increased by approximately $220 \%, 150 \%$ and $100 \%$, respectively.

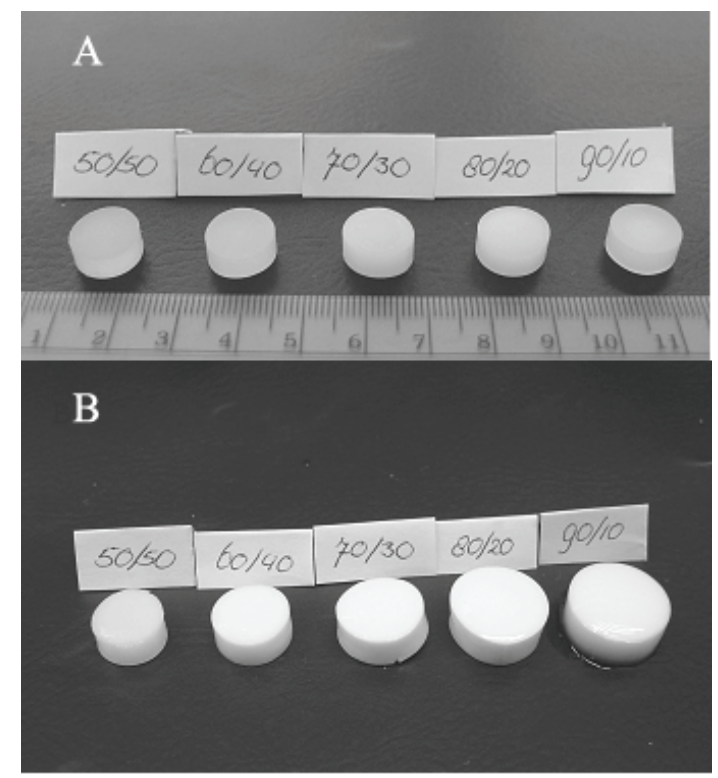

Figure 2.3A. Samples of the different Slipskin ${ }^{\circledR}$ formulations in dry form. B. Samples of the different Slipskin ${ }^{\circledR}$ formulations after equilibrating in water at room temperature.

\subsubsection{Fluorescein release}

Fluorescein concentrations, measured after release from coils coated with 90-10-F, 80-20-F, 70-30-F, or 60-40-F are plotted in Figure 2.4. All coils show fast initial release. The concentrations tend to stabilize after appr. $12 \mathrm{~h}$, at a plateau values which relate to the hydrophilicity of the coating. The most hydrophilic coating (90-10-F) and the most hydrophobic coating (60-40-F) correspond with plateau concentrations of appr. $6 \mu \mathrm{M}$ and 3 $\mu \mathrm{M}$, respectively. The results suggest that, in the plateau phase, more of the dye remains 
entrapped in a relatively hydrophobic coating. The same conclusion was reached in our previous work on the release of rhodamine from Slipskin ${ }^{\circledR}$-coated coils [18].

To quantify how much of the dye remains entrapped in the different coatings after $24 \mathrm{~h}$, we also immersed 90-10-F, 80-20-F, 70-30-F and 60-40-F coated coils in 96\% alcohol, instead of Tris buffer. The alcohol is known to dissolve the coatings, or at least to cause extensive swelling. Both situations were assumed to correspond with quantitative release of the dye. By comparing the fluorescein concentrations after immersion in alcohol $(24 \mathrm{~h})$ with those after immersion in Tris buffer $(24 \mathrm{~h})$, we calculated that the percentages of released dye decrease in the order $60 \%, 53 \%, 40 \%$, and $35 \%$ for the coatings $90-10-\mathrm{F}, 80-20-\mathrm{F}, 70-30-\mathrm{F}$ and $60-40-\mathrm{F}$, respectively.

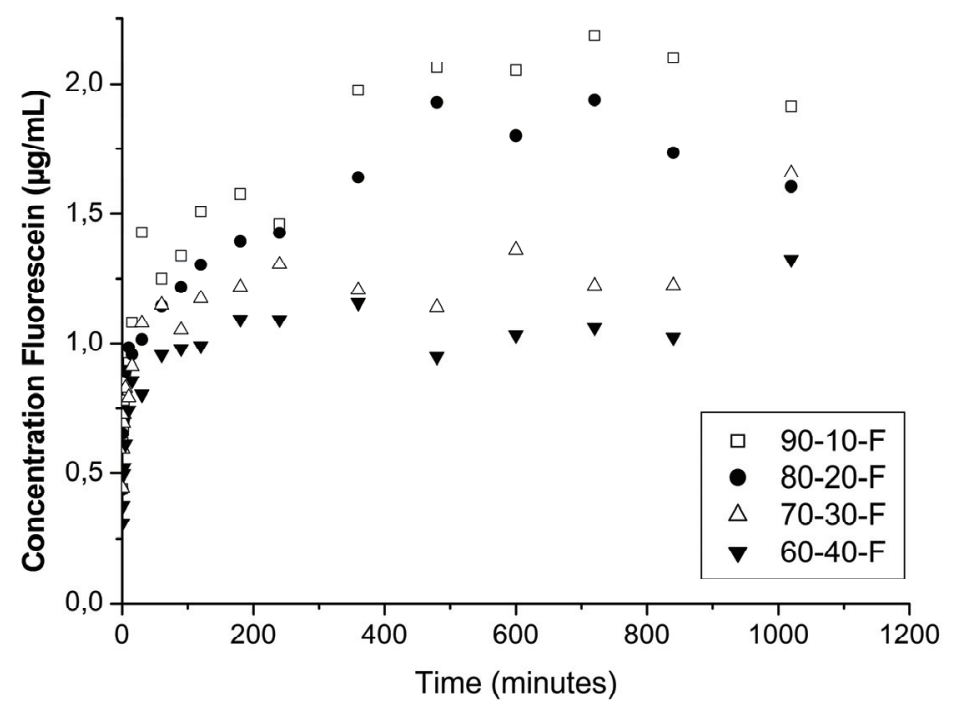

Figure 2.4. Cumulative in vitro release curves of fluorescein from four different Slipskin ${ }^{\circledR}$ coatings.

In another set of experiments the coils with the coating $80-20-\mathrm{F}$ were cut in pieces of $16-\mathrm{mm}$ in length. The ends of these coils were carefully inspected for the absence of any sharp edges. These coils were quickly dipped in $70 \%$ alcohol, dried, and introduced by an experienced ophthalmologist under the lower eyelid of a healthy human volunteer. Subsequently, fluorescence photographs of his eye were taken. Figure 2.5, taken appr. 30 minutes after introduction of the coil, shows that the dye is indeed spread over the tear film. The presence of the coil did not irritate the volunteer.

Although the fluorescein that was released in the tear fluid of the volunteer could be clearly monitored, we realize that the amount of released dye is very small. We therefore asked 
ourselves the question how the capacity of the device could be increased. The answer lies, in our opinion, in utilization of the inner lumen as a reservoir for the dye, drug, or antibiotic. So far, the lumen of the coils was essentially unused space. Filling this space with either the drug in pure form, a solution of the drug, or a mixture of the drug and (for example) a hydrogel, can dramatically increase the capacity of the device. If both ends of the coil are closed, release of the drug requires radial diffusion, from the reservoir to the tear fluid, following a track in between adjacent windings of the coil. This implies a coupled kinetic mechanism: initial quick release from the surface of the device (as described above), followed by sustained release from the relatively large reservoir in the lumen of the coil.

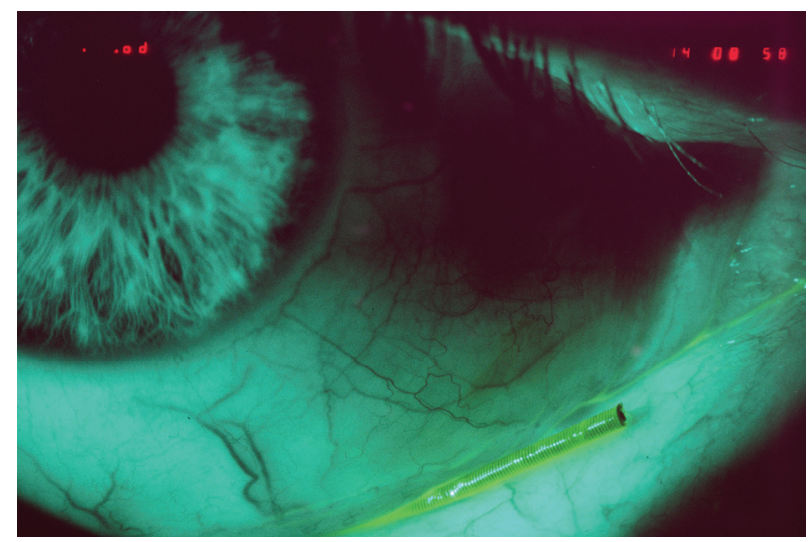

Figure 2.5. A photograph of the device in the conjunctival sac. The lower eyelid was pulled forward in this situation; the coil is normally invisible.

We did a limited number of initial experiments on filling the coil's lumen with an extra amount of fluorescein dye. This showed, almost immediately, that filling the lumen causes problems in terms of the flexibility of the coil, which is a conditio sine qua non for the intended application. It is mandatory that the coil bends easily in order to adopt a curved shape which aligns with the sclera under the lower eyelid.

We found that filling the lumen with fine fluorescein powder was technically very difficult, and this presumably holds for all powders. Filling the lumen with an aqueous solution of the dye lead to sticking of adjacent windings of the coil, which also translated in stiffness. We found a non-optimal solution to this problem in the following manner: in the lumen of the coil, several pieces of a straight metallic wire were inserted (Figure 2.6). The short wires had the same diameter as the wire from which the coil is made. The short wires also had a SlipSkin ${ }^{\circledR}$ coating, but the difference with the coiled wire is that the coating is thicker, and richer in the drug. 


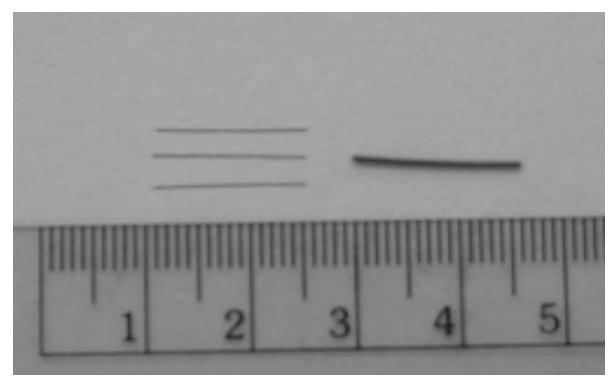

Figure 2.6. An illustration of the build-up of the device. The Slipskin ${ }^{\circledR}$ coated coil is shown on the right. Three straight wires with a relatively thick coating are shown on the left. The three straight wires are placed inside the coil's lumen and both ends are then sealed with a polymeric cap.

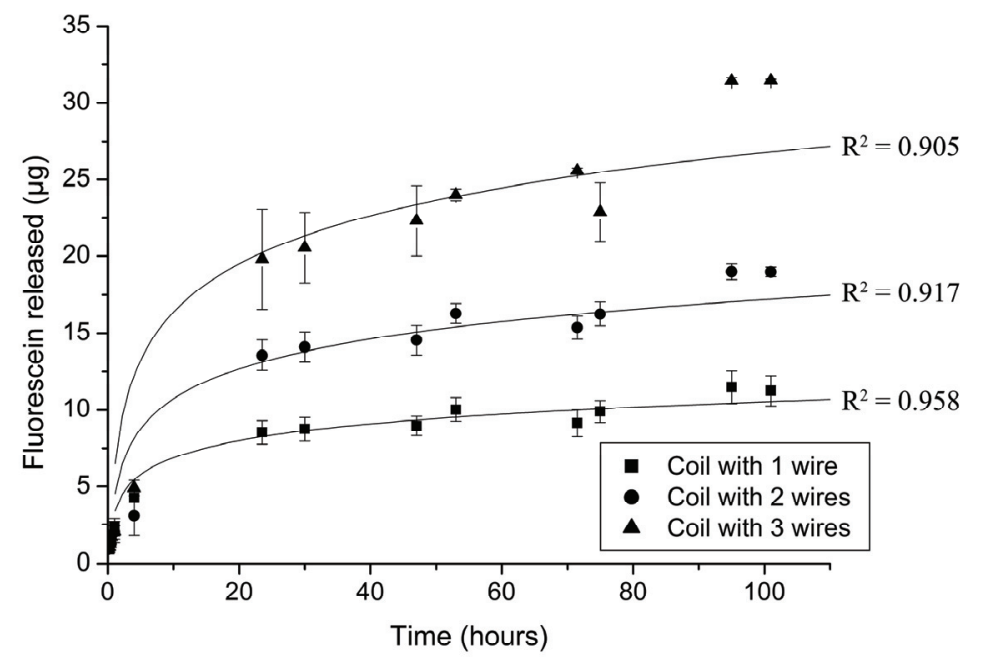

Figure 2.7. Cumulative release curves of the fluorescein in vitro. The wires inside the lumen increased the capacity of the device.

For example, a series of three different fluorescein-charged filled devices was made. Two different wires were used. The first wire (1f) had the SlipSkin ${ }^{\circledR} 70-30-F$ coating (Table 2.2); this coating is loaded with fluorescein in the ratio $\operatorname{SlipSkin}^{\circledR}:$ dye $=100: 1$ (mass : mass). The second wire (2f) had a SlipSkin ${ }^{\circledR} 70-30$ coating (thickness $3 \mu \mathrm{m}$ ), charged with fluorescein in the ratio SlipSkin ${ }^{\circledR}:$ dye $=1: 1$ (mass : mass). A coil out of wire $1 \mathrm{f}$ was made (coiled length 16 $\mathrm{mm}$ ), and one, two, or three straight pieces of wire $2 \mathrm{f}$ (length $12 \mathrm{~mm}$ ) were introduced in the lumen. The results of the initial release experiments in vitro are shown in Figure 2.7. Note that the release curves show a strong dependency on the number of straight wires inside the coil's lumen. These data confirm that filling the lumen increases the capacity of the device. 
Moreover, the data suggest that radial diffusion of the dye, in between adjacent windings, indeed occurs.

\subsubsection{Mydriasis}

The in vivo pilot experiment, which demonstrated fluorescein release in the tear film of a volunteer, prompted us to do an analogous experiment with atropine-charged coils, to try and see whether mydriasis (pupil-widening) could be obtained. Note that these coils carried the coating 90-10-A (Table 2.2), and had empty lumens. Two volunteers were recruited, and they both received a coil under their right eyelid. Unfortunately, no pupil-widening was found, presumably because of too low dosage. Then, we decided to place two coils simultaneously under the left eyelid of volunteer II. This invoked clear mydriasis after several minutes, and the effect was found to persist for $>2 \mathrm{~h}$. Figure 2.8 shows photographs of the left eye of volunteer II, before and after insertion of the two atropine-charged coils. Noteworthy, the presence of two coils in the left eye did not cause irritation or discomfort to volunteer II.
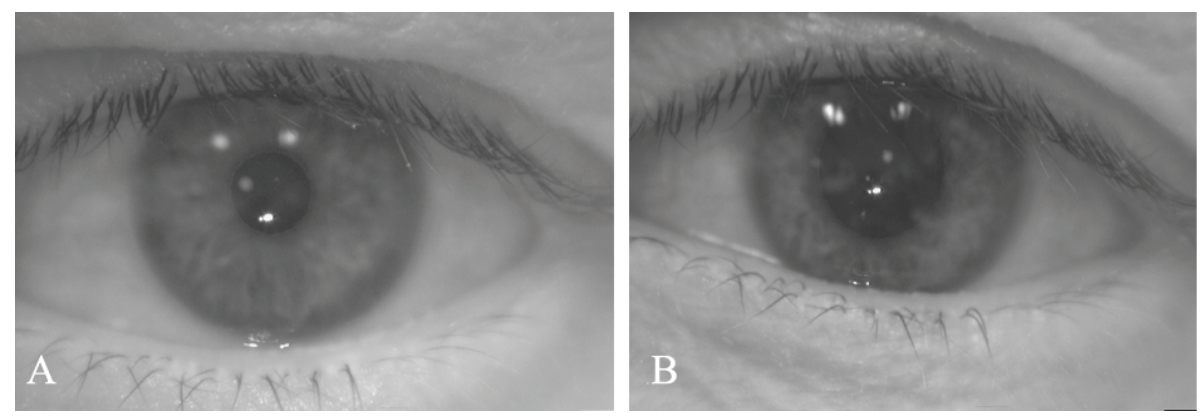

Figure 2.8. A. Control situation, prior to the insertion of the atropine-releasing coil(s). B. Mydriasis, caused by atropine which is released from two coils simultaneously.

\subsubsection{Antibiotic release}

So far, our experiments with Slipskin ${ }^{\circledR}$-coated coils for controlled local delivery of antibiotic agents have been of a preliminary nature. Only the antibiotics chloramphenicol and gentamicin were used, only Slipskin ${ }^{\circledR}$ 70-30 was used as the platform, and only in vitro experiments, directed at measuring zones of inhibition in E. coli cultures, are reported here.

Experiments were done with two types of lumen-filled and chloramphenicol-charged coils, which will be referred to as coils A and coils B. Again, two different coated wires were used. The first (1c) had the 70-30-C coating, i.e. the SlipSkin ${ }^{\circledR}$ : antibiotic ratio was $1: 1$ (mass : mass; viz. Table 2), with thickness $2 \mu \mathrm{m}$ (2 layers). The second wire (2c) had the same coating, but with thickness $5 \mu \mathrm{m}$ (4 layers). Coil A had an exterior of wire 1c, (coiled length $16 \mathrm{~mm}$ ), 
and three short wires $2 \mathrm{c}$ (length $12 \mathrm{~mm}$ ) in the lumen. Coil B had an exterior of wire 2c (coiled length $16 \mathrm{~mm}$ ), and also three short wires $2 \mathrm{c}$ (length $12 \mathrm{~mm}$ ) in the lumen. The total amount of antibiotic was calculated to be $100 \mu \mathrm{g}$ in coil A, and $260 \mu \mathrm{g}$ in coil B. The zone-of-inhibition assay was positive for both coils (Figure 2.9), and it was also clear that the inhibitory activity of coil B exceeded that of coil A. As a reference, coil C was prepared without any chloramphenicol present in the coating or lumen. The figure shows that coil $\mathrm{C}$ did not show any antibiotic activity.

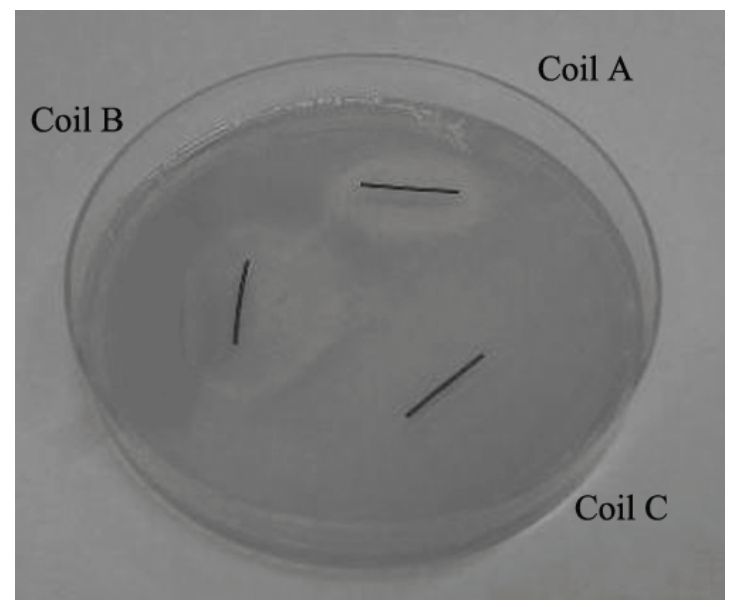

Figure 2.9. Inhibition of the bacteria in vitro, caused by chloramphenicol which is released from coil A and coil B. Coil C, without any drug present, was used as a reference. The data reflect that coil $A$ contained less chloramphenicol than coil B.

\subsection{Discussion}

The most important advantages of our approach are: (i) accurate control over the thickness of the drug-loaded coating and, hence, accurate control over the amount of drug that is introduced into the eye; (ii) ease of insertion and removal of the coils from the eye. The most important remaining questions may be:

1. Does the presence of the spiral under the eye lid put the patient at risk? Possible complications are irritation, or even corneal erosion. It is clear that any of such risks are unacceptable.

2. What is the best method to increase the capacity of the device?

3. Is it possible to steer the release kinetics in order to accomplish controlled administration of the drug to the eye over a longer time-interval (e.g., one week)? 
The first question is, clearly, difficult to answer. Our volunteers have not experienced any discomfort during or after wearing the spiral, but obviously this does not allow us to draw any conclusions. We plan to start a series of animal experiments in an attempt to quantify the degree of irritation that may be caused by a spiral. In this context, it may be important to mention that we made some progress regarding the sealing of the coils on both ends. In catheter industry, techniques are available to round off sharp ends of coils and tubes by means of a photo-reactive glue. Recently, this technology was successfully applied to our coils (Figure 2.10).

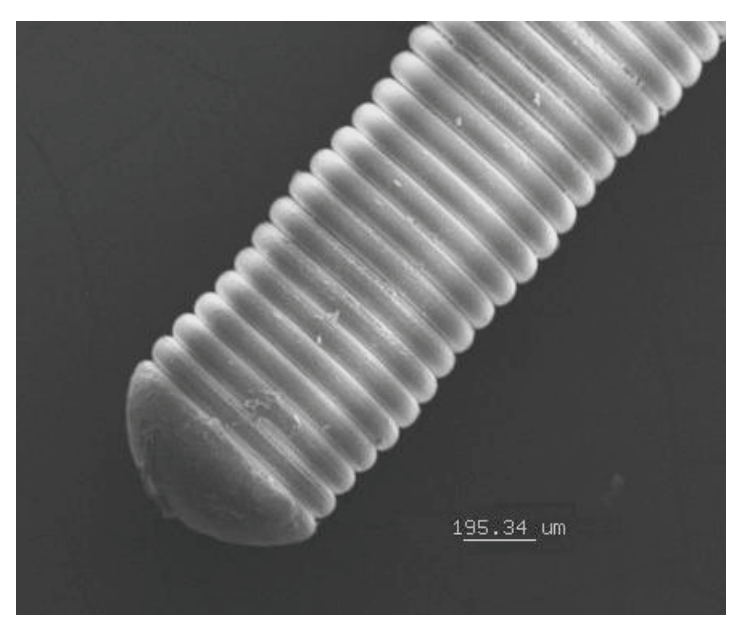

Figure 2.10. Scanning electron micrograph of one of the ends of the coil. The ends were sealed with cyanoacrylate to close the lumen reservoir and to avoid sharp edges.

Our present approach for using the lumen as a depot for the drug or antibiotic to be released is clearly non-optimal. The use of short straight metallic wires with a relatively thick hydrophilic coating with a relatively high content of the drug is, most likely, a non-optimal solution. Filing the lumen with the drug as a powder proved technically difficult, at least in our hands. A better solution to this problem may be found through the use of fibers consisting of a hydrophilic polymer, and high amount of the drug embedded therein. Further work along this line is currently in progress in our laboratory.

The answer to the third question is clearly dependent on the method for filling the lumen. All parameters affecting the release kinetics will be defined in the future. One of these is, undoubtedly, the manufacture of the coils, i.e. the method of winding. If adjacent winding are pressed onto each other, the release is likely to be much slower in comparison with more loosely wound coils. 


\subsection{Conclusion}

Controlled local release of a dye, a drug, or an antibiotic agent from the surface of SlipSkin ${ }^{\circledR}$ coated coils seems technically feasible, but significant problems still exist, particularly with respect to the application of drug-charged coils for drug delivery to the front side of the eye. Further research and development work is necessary to ensure that this approach, when used for delivery of drugs to the eye, does not put the patient at risk. Moreover, it must be explored which are the most important indications for use of this drug delivery method. These may concern short time delivery of drug (e.g., mydriatic drugs prior to corneal surgical procedures), or long-term delivery of, for instance, antibiotics or anti-inflammatory agents. In both situations, the use of the delivery device may prove a useful alternative to repeated administration of drugs via eye drops, since it may be more convenient (to patient, nurse and physician), and cost-effective.

\section{References}

1. Montdargent B., and Letourneur D. Toward new biomaterials. Infect Control Hosp Epidemiol 21 (6) (2000) 404-410.

2. Wang C., Zhang Q., Uchida S., and Kodama M. A new vascular prosthesis coated with polyamino-acid urethane copolymer (PAU) to enhance endothelialization. J Biomed Mater Res 62 (3) (2002) 315-322.

3. Mueller X.M., Jegger D., Augstburger M., Horisberger J., and von Segesser L.K. Poly2methoxyethylacrylate (PMEA) coated oxygenator: an ex vivo study. Int J Artif Organs 25 (3) (2002) 223-229.

4. Lee J.H., and Oh S.H. MMA/MPEOMA/VSA copolymer as a novel blood-compatible material: effect of PEO and negatively charged side chains on protein adsorption and platelet adhesion. $J$ Biomed Mater Res 60 (1) (2002) 44-52.

5. $\quad$ Court J.L., Redman R.P., Wang J.H., Leppard S.W., Obyrne V.J., Small S.A., Lewis A.L., Jones S.A., and Stratford P.W. A novel phosphorylcholine-coated contact lens for extended wear use. Biomaterials 22 (24) (2001) 3261-3272.

6. Vacheethasanee K., and Marchant R.E. Surfactant polymers designed to suppress bacterial (Staphylococcus epidermidis) adhesion on biomaterials. J Biomed Mater Res 50 (3) (2000) 302-312.

7. Defife K.M., Hagen K.M., Clapper D.L., and Anderson J.M. Photochemically immobilized polymer coatings: effects on protein adsorption, cell adhesion, and leukocyte activation. J Biomater Sci Polym Ed 10 (10) (1999) 1063-1074.

8. Hanssen H.J., and Koole L.H. Heparin-releasing intravascular guide wires. Med Device Technol 13 (7) (2002) 20-22.

9. Prokop A., Kozlov E., Nun Non S., Dikov M.M., Sephel G.C., Whitsitt J.S., and Davidson J.M. Towards retrievable vascularized bioartificial pancreas: induction and long-lasting stability of polymeric mesh implant vascularized with the help of acidic and basic fibroblast growth factors and hydrogel coating. Diabetes Technol Ther 3 (2) (2001) 245-261.

10. Park S., Bearinger J.P., Lautenschlager E.P., Castner D.G., and Healy K.E. Surface modification of poly(ethylene terephthalate) angioplasty balloons with a hydrophilic poly(acrylamide-co-ethylene glycol) interpenetrating polymer network coating. J Biomed Mater Res 53 (5) (2000) 568-576. 
11. Altankov G., Thom V., Groth T., Jankova K., Jonsson G., and Ulbricht M. Modulating the biocompatibility of polymer surfaces with poly(ethylene glycol): effect of fibronectin. J Biomed Mater Res 52 (1) (2000) 219-230.

12. Wieneke H., Sawitowski T., Wnendt S., Fischer A., Dirsch O., Karoussos I.A., and Erbel R. Stent coating: a new approach in interventional cardiology. Herz 27 (6) (2002) 518-526.

13. Babapulle M.N., and Eisenberg M.J. Coated stents for the prevention of restenosis: Part II. Circulation 106 (22) (2002) 2859-2866.

14. Drachman D.E., Edelman E.R., Seifert P., Groothuis A.R., Bornstein D.A., Kamath K.R., Palasis M., Yang D., Nott S.H., and Rogers C. Neointimal thickening after stent delivery of paclitaxel: change in composition and arrest of growth over six months. J Am Coll Cardiol 36 (7) (2000) 2325-2332.

15. Sousa J.E., Costa M.A., Sousa A.G., Abizaid A.C., Seixas A.C., Abizaid A.S., Feres F., Mattos L.A., Falotico R., Jaeger J., Popma J.J., and Serruys P.W. Two-year angiographic and intravascular ultrasound follow-up after implantation of sirolimus-eluting stents in human coronary arteries. Circulation 107 (3) (2003) 381-383.

16. Drachman D.E. Clinical experience with drug-eluting stents. Rev Cardiovasc Med 3 (5) (2002) 31-37.

17. Morice M.C. A new era in the treatment of coronary disease? Eur Heart J 24 (3) (2003) 209-211.

18. Hanssen H.H., Wetzels G.M., Benzina A., van der Veen F.H., Lindhout T., and Koole L.H. Metallic wires with an adherent lubricious and blood-compatible polymeric coating and their use in the manufacture of novel slippery-when-wet guide wires: possible applications related to controlled local drug delivery. J Biomed Mater Res 48 (6) (1999) 820-828.

19. Peerlings C.C., Hanssen H.H., Bevers R.T., Boelen E.J., Stelt B.J., Korthagen E.J., and Koole L.H. Heparin release from slippery-when-wet guide wires for intravascular use. J Biomed Mater Res 63 (6) (2002) 692-698.

20. Hanssen J.H.L., and Koole L.H. Wire for medical use coated with polyethersulphone and a copolymer. US6086547 (2000).

21. Koole L.H., Hanssen J.H.L. Wire, tube or catheter with hydrophilic coating. US2003/006078 (2003).

22. Merck Index, entry \#891, 11th ed., Merck \& Co. Inc., Rahway, NJ, USA (1989) 138.

23. Merck Index, entry \#2068, 11 th ed., Merck \& Co. Inc., Rahway, NJ, USA (1989) 318. 


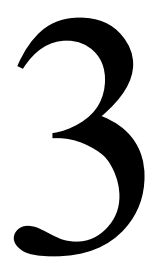

\section{Studies on a new device for drug delivery to the eye}

Rachel T. Pijls, Trudi Sonderkamp, Gert W. Daube, Ralph Krebber, Hans H.L. Hanssen, Rudy M.M.A. Nuijts and Leo H. Koole. Adapted from: European Journal of Pharmaceutics and Biopharmaceutics 59 (2005) 283-288 


\section{Abstract}

Delivery of drugs to the anterior side of the eye is routinely done with eye drops, but this method results in low bioavailability and low patient compliance. Herein, we describe a new device for the delivery of drugs to the eye. The device, called the OphthaCoil, consists of a drug-loaded adherent hydrogel coating on a thin metallic wire, which is coiled. The drug release of the dye fluorescein and the antibiotic chloramphenicol have already been evaluated in vitro. In this report the drug release of the anti-infective pradofloxacin was evaluated in vitro and in vivo. The data show that the OphthaCoil is capable of sustained drug delivery to the tear film in dogs. Drug levels in the tear fluid of the dogs were well above the MIC-values of relevant bacteria after $16 \mathrm{~h}$, but it should be noted that pradofloxacin has an exceptionally high antimicrobial activity. The study indicates that the OphthaCoil holds promise as a platform for sustained release of drugs to the eye. The device was well tolerated, but the devices were lost when left overnight. Most probably, this is due to the third eyelid pushing the device out of the conjunctival fornix during sleep. It should be noted that this complication has no immediate implication for extended wear of the OphthaCoil in humans, as humans do not have third eyelids. 


\subsection{Introduction}

Hydrogels are three-dimensional, hydrophilic, polymeric networks capable of taking up large amounts of water or biological fluids [1]. The networks are composed of homopolymers or copolymers and are insoluble due to the presence of chemical or physical cross-links. The physical properties of hydrogels make them attractive for a variety of biomedical applications [2-6]. Hydrogels are especially suitable as platforms for controlled local delivery of drugs. In the simplest form, a drug is impregnated in a dry hydrogel matrix. When implanted, the matrix will swell (uptake of water from the environment), with concomitant diffusion-controlled release of the drug. More sophisticated strategies may be based on stimuli-responsive hydrogels. For example, $\mathrm{pH}$-sensitive hydrogels have been devised for insulin delivery [2]. The role of hydrogels as drug delivery vehicles has been highlighted in several recent reviews [1-3]. In this article, we specifically focus on the use of hydrogels for administration of drugs to the anterior side of the eye. Delivery of drugs to the tear film is routinely done with eye drops, which are well-accepted and for most patients easy to use. However, most of the solution in each eye drop is blinked out or drained into the nasal cavity. This leads to poor bioavailability of the drug [7]. Frequent administrations are required to maintain therapeutic levels, resulting in low patient compliance [8]. Development of an alternative to eye drops via sustained delivery of a drug to the tear film is a major challenge. Hence, numerous studies have been devoted to developing devices for sustained drug delivery to the tear film; all of these are based on hydrogel technology [7-14]. This work resulted in a few commercial products (e.g. Ocusert ${ }^{\mathbb{B}}$ [10]). Several other concepts are still under investigation, e.g. a mucoadhesive ocular insert based on thiolated poly(acrylic acid) [8], Mydriasert ${ }^{\circledR}$ [9], and a Gelfoam ${ }^{\circledR}$ based ocular device [7].

In general, commercialization of ocular inserts for drug delivery has proven troublesome, probably in part due to psychological factors. Doctors and patients generally feel more comfortable with traditional eye drops. Use of hydrogels for controlled drug delivery to the tear film is often associated with the complication that the insert becomes weak during the process of swelling and drug release. This may hamper the removal of the insert when drug release is completed. This complication has been described for a mucoadhesive minitablet [15]. It should be noted, that biodegradable matrices for drug delivery to the tear film have also been described. In theory, these have the advantage that no removal is necessary, due to enzymatic or chemical degradation. However, biodegradable inserts may also have disadvantages; erosion or disintegration into smaller pieces may result in occasional blurring of vision. The 
disintegrated insert may also move around the ocular surface causing irritation and might be easily lost.

Herein, we describe a new device for controlled delivery of drugs to the eye, which is also based on hydrogel technology. The major difference between the new device and its predecessors is that a thin hydrogel coating is used; this coating is a drug-loaded adherent hydrogel on a thin stainless steel wire, which forms a short coil. The new device, which is called OphthaCoil, is shown in Figure 3.1.

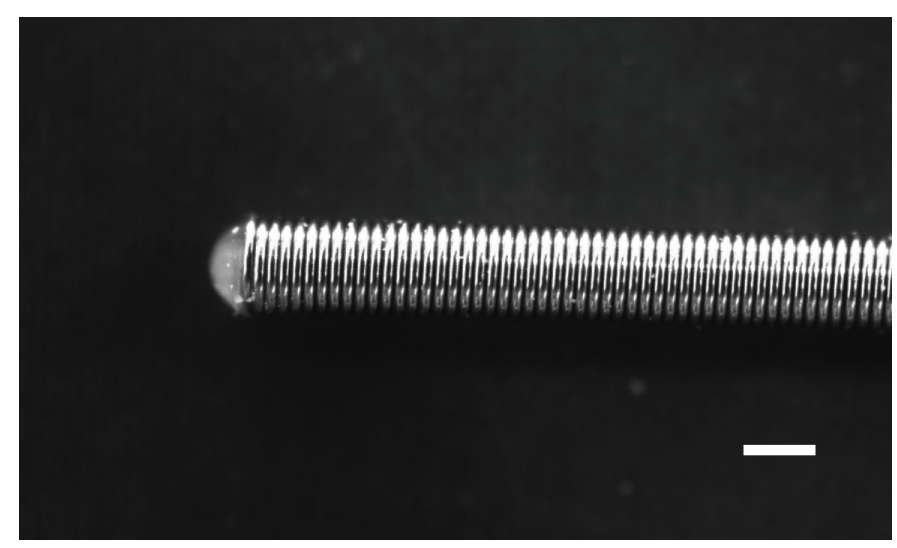

Figure 3.1. Photograph showing the OphthaCoil ( $\mathrm{bar}=0.5 \mathrm{~mm}$ ). The adjacent windings of the coiled structure, as well as one of the polymeric caps, closing the inner lumen, are clearly visible. The outer diameter of the coil is $600 \mu \mathrm{m}$; the diameter of the coated coiled wire is app. $90 \mu \mathrm{m}$.

The OphthaCoil has several characteristic features:

- The device consists of a flexible coil, which has a drug loaded biocompatible coating. The coil is inserted into the lower conjunctival sac, i.e. behind the lower eyelid.

- The ends of the coil are closed with a spherical cap to avoid sharp edges.

- The device has an inner lumen, which is used as a second drug reservoir. Drug molecules can escape from the lumen towards the tear fluid via diffusion in between adjacent windings of the coil; in principle, this allows long-term drug release.

- After swelling of the coating and complete release of the drug, the device retains its integrity.

Release of drugs from thin coatings on wires and coils has been previously studied by our group [16-20]. In all cases, we used co-polymers of N-vinyl pyrrolidone (NVP) and butyl methacrylate (BMA) as the coating. Mechanistic studies were done with the help of impregnated dyes and the antibiotic chloramphenicol [16-20]. Based on the in vitro release data, it was anticipated that a short coil as shown in Figure 3.1 can contain and release 
substantial amounts of a drug, i.e. sustained release using the OphthaCoil should, in principle, be possible. It is a conditio sine qua non, however, that the OphthaCoil is well tolerated in the eye, i.e. the device should not cause any irritation or inconvenience to the patient.

Presently, we report the results of our first tests on the tolerance of the OphthaCoil in an in vivo model. OphthaCoils, charged with pradofloxacin, a new fluoroquinolone developed by Bayer HealthCare AG (Monheim, Germany) exclusively for veterinary medicine, were used. The chemical structure of pradofloxacin is given in Figure 3.2. First, the release of pradofloxacin in vitro was studied. Then, OphthaCoils charged with pradofloxacin were placed in the eyes of six Beagle dogs. The animals were examined, samples of the tear fluid were taken, and the concentration of the antibiotic was monitored as a function of time.

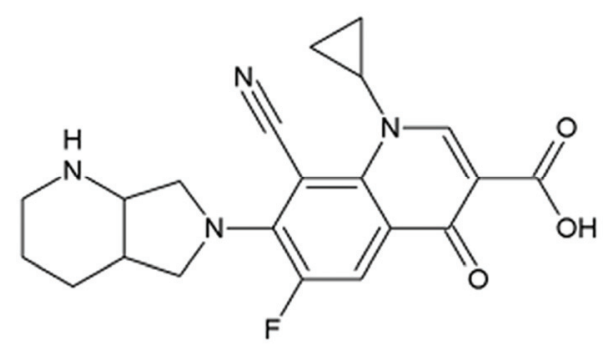

Figure 3.2. The chemical structure of pradofloxacin.

\subsection{Materials and Methods}

\subsubsection{The OphthaCoils}

The hydrogel used is the co-polymer of the hydrophilic monomer NVP and the hydrophobic monomer BMA. A molar ratio of 70:30 (NVP:BMA) was chosen, because this hydrophilicity offers a balance between the degree of swelling and the drug release. The co-polymer was prepared in a free radical bulk polymerization reaction, using $\alpha, \alpha^{\prime}$-azoisobutyronitrile (AIBN) as the initiator. The procedure resulted in the desired co-polymer as a glassy, opaque rod. The rod was cut into pieces, which were dissolved in N-methyl pyrrolidone (NMP) by mechanical stirring overnight.

A thin stainless steel wire with a diameter of $76 \mu \mathrm{m}$, was first coated with a thin layer of a binding polymer (poly(ethersulfone), thickness $1 \mu \mathrm{m}$ ), and then coated twice with a polymer solution consisting of $220 \mathrm{~mL}$ NMP, $15 \mathrm{~g}$ of the co-polymer NVP:BMA 70:30, and $10 \mathrm{~g}$ of pradofloxacin (Bayer HealthCare AG, Monheim, Germany). An extrusion-like coating procedure, as described before $[18,19]$, was used. The thickness of the coating layer was measured online with a laser system and afterwards it was verified with scanning electron microscopy. 
Half of the wire was stored (wire A); this wire had a coating thickness of $3.75 \mu \mathrm{m}$. The other half was processed twice more (four passages in total), resulting in a drug-containing hydrogel coating with a thickness of $7.25 \mu \mathrm{m}$ (Wire B). Wire A was coiled around a core wire of 432 $\mu \mathrm{m}$ thickness, and the resulting coil was cut into pieces of $16 \mathrm{~mm}$. Wire B was cut into pieces of $15 \mathrm{~mm}$ and three of these wires were put into the coil. The coils were carefully closed with a photo reactive glue, using a technique which is well-known in the catheter industry. The caps effectively prevented sharp edges, see Figure 3.1. The dimensions of the coils were $16 \mathrm{~mm}$ long and appr. $0.6 \mathrm{~mm}$ in diameter.

Based on several known parameters, it is possible to calculate the total charge of pradofloxacin on each OphthaCoil. These parameters are: length of wire A $(35 \mathrm{~cm})$, thickness of the pradofloxacin-containing coating of wire $\mathrm{A}(3.75 \mu \mathrm{m})$, length of wire $\mathrm{B}(15 \mathrm{~mm})$, thickness of the pradofloxacin-containing coating of wire $\mathrm{B}(7.25 \mu \mathrm{m})$ and the concentration of pradofloxacin in the hydrogel coating (40\%). This resulted in an amount of $135.6 \mu \mathrm{g}$ pradofloxacin on the coil (wire A) and $3 \times 11.7 \mu \mathrm{g}=35.1 \mu \mathrm{g}$ on wires B. The calculated total charge of pradofloxacin was then $170.7 \mu \mathrm{g}$ per OphthaCoil. It is worth noting that wire B has a contribution of only $20 \%$ to the capacity of the device.

\subsubsection{In vitro release experiments}

Pradofloxacin-charged OphthaCoils were immersed in $1 \mathrm{~mL}$ sterile PBS (phosphate buffered saline) that was refreshed at the following time-points: 5, 15, $30 \mathrm{~min}, 1,3,6,12,24$ and $48 \mathrm{~h}$. The pradofloxacin concentrations in these solutions were determined with a zone-of-inhibition assay [21]. Plastic Petri dishes were filled with Iso-sensitest ${ }^{\mathrm{TM}}$ medium which was used as culture medium. E. coli 14 was used as indicator bacterium, because E. coli infections are common in the eyes of dogs. After coagulation and drying of the culture medium, the indicator bacterium was brought onto the medium and holes were punched with a radius of $4 \mathrm{~mm}$ at equal distance in the agar bottom. These holes were filled with $0.1 \mathrm{~mL}$ of the solutions with the released pradofloxacin. Other holes were filled with $0.1 \mathrm{~mL}$ of a dilution series, so a calibration curve could be made to determine the concentration of pradofloxacin in the measurements. After $24 \mathrm{~h}$ of incubation at $37^{\circ} \mathrm{C}$ the diameters of the inhibition zones were measured and the concentrations were calculated.

\subsubsection{Tolerance in vivo}

The tolerance and safety study was done with three female adult Beagle dogs, and one OphthaCoil per animal. In these experiments, the hydrogel coating did not contain any active substance. The dogs were about 2 years old and they weighed between 10.9 and $12.4 \mathrm{~kg}$. The 
animals were housed individually in cages with tiled floor and identification was assured through an individual, unchangeable ear tattooing provided by the breeder. The relative humidity varied between $50-60 \%$ and the temperature varied between $15-21^{\circ} \mathrm{C}$. Lighting was controlled to ensure approximately $12 \mathrm{~h}$ of artificial light from $06.00 \mathrm{a} . \mathrm{m}$. to $06.00 \mathrm{p} . \mathrm{m}$. Once a day the dogs were fed a commercially available dry dog food and water was provided for $a d$ libitum consumption.

To avoid unwanted defense reactions during application of the coil, one drop of the local anaesthetic oxbarucain (Oxbarukain ${ }^{\circledR}$ uno, Chauvin Ankerpharm GmbH, Rudolstadt, Germany) was administered to the eye. The anaesthetic was administered five minutes before application of the coil. Oxbarucain is known to be active for about $30 \mathrm{~min}$. The coils were placed in the lower conjunctival sac of each dog's left eye. The right eyes served as controls. The dogs were continuously observed for $8 \mathrm{~h}$. Then, the presence and the position of the coils were checked. The next day, after $24 \mathrm{~h}$, the coils were checked again and were removed when still present in the eye.

Before the application, and after the removal of the coil, an ophthalmologic examination took place, where both eyes were examined for alterations of conjunctiva, third eyelid, cornea and sclera. The following parameters were examined: the ocular discharge, the third eyelid, the cloudiness and surface of the cornea, the redness, secretion and swelling of the conjunctiva, the sclera and the behavior of the dog. The results were compiled in a four stage grading system (normal; + slight change; ++ moderate change; +++ severe change).

\subsubsection{In vivo release experiment}

The kinetic study was done with a group of six female Beagle dogs and one pradofloxacincharged OphthaCoil per animal. All dogs were between $1 \frac{1}{2}$ and $2 \frac{1}{2}$ years old. Their weight ranged between 10.9 and $13.9 \mathrm{~kg}$. The animals were housed individually in cages, as in the previous experiment. Before application of the coil a tear fluid sample was taken with a Schirmer test strip (Liposic, dr Mann Pharma, Berlin, Germany) and again one drop of oxbarucain was administered five minutes before application of the coil. Tear fluid samples were taken at the following time-points: after 1, 2, 4, 6 and $8 \mathrm{~h}$. The dogs were continuously observed and after $8 \mathrm{~h}$ the coil was removed and stored in an Eppendorf vial at $4{ }^{\circ} \mathrm{C}$. The next day the same coils were inserted and tear fluid samples were taken again after 1, 2, 4, 6 and 8 $h$. Then the coil was left in the eye to check the presence and position of the coil overnight.

The day before the application of the coil and on the last day after the removal of the coil, an ophthalmologic examination took place, where both eyes of the animals were examined, as 
mentioned in Section 3.2.3. The concentrations of pradofloxacin in the tear fluid samples were determined by HPLC and tandem mass spectroscopy [22].

\subsection{Results and Discussion}

\subsubsection{Build-up of the OphthaCoils}

The OphthaCoil consists of a coil (wire A) with three shorter pieces of a wire with a thicker coating thickness (wire B) inside. This is schematically shown in Figure 3.3.

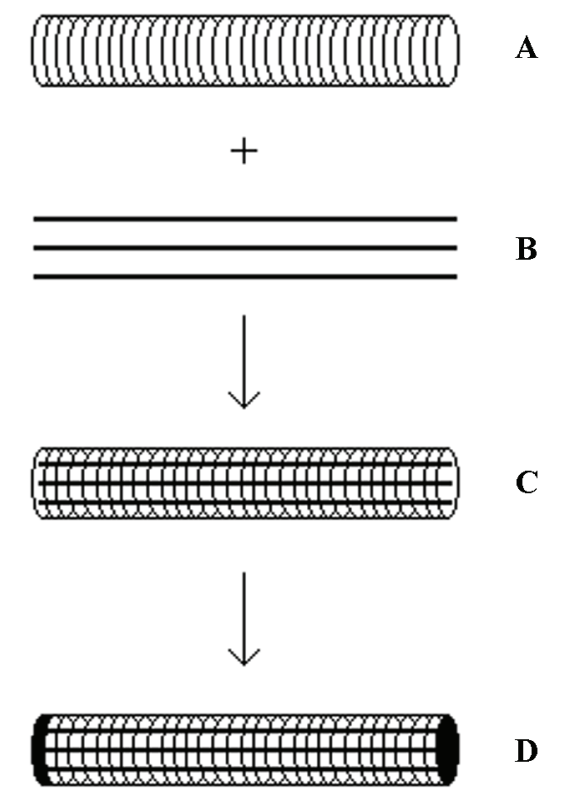

Figure 3.3. Schematic view of the OphthaCoil manufacturing: (A) Coil of wire A; (B) Three straight pieces of wire B; (C) Wires B placed in the interior of the coil; (D) Closing the ends by a polymeric cap.

\subsubsection{Release of pradofloxacin in vitro}

Figure 3.4 shows the results of the release experiments in vitro. The concentration of the released pradofloxacin is plotted as a function of time. The initial release is fast, which is in agreement with our previous observations on the release of, e.g. rhodamine and heparin from comparable coils [16-18]. At the end of the experiment, i.e. after $24-48 \mathrm{~h}$ of immersion, a released pradofloxacin concentration of approximately $0.6 \mu \mathrm{g} / \mathrm{mL}$ was found in both cases. This concentration is still far above the MIC-values, which are $0.015-0.03 \mu \mathrm{g} / \mathrm{mL}$ for $E$. coli 
ATCC 8739 and 0.03-0.06 $\mu \mathrm{g} / \mathrm{mL}$ for Staphylococcus aureus ATCC 6538 [23]. This implies that the OphthaCoil shows sustained release of the anti-infective in vitro, at least for $48 \mathrm{~h}$.

The total amount of pradofloxacin released after $48 \mathrm{~h}$ was calculated to be 32 and $35 \mu \mathrm{g}$. This implies that approximately $20 \%$ of the anti-infective, that was present in and on the device at the start of the experiment, is actually released. Most likely, release of small quantities of the anti-infective goes on after $48 \mathrm{~h}$, although we also anticipate that a substantial fraction of active species remains entrapped in the hydrogel coating, even after swelling to complete equilibrium [18].

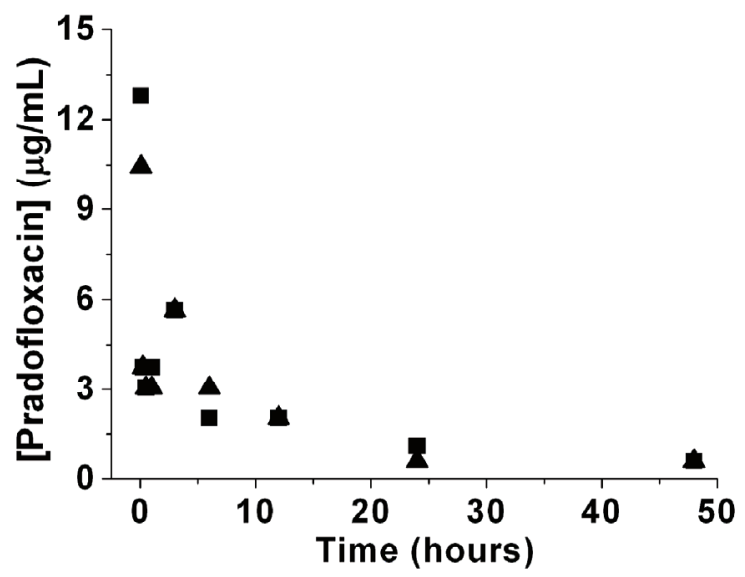

Figure 3.4. The release of pradofloxacin in vitro $(n=2)$. While the initial release is fast, concentrations after $48 \mathrm{~h}$ are significantly higher as compared to the MIC-value of pradofloxacin.

\subsubsection{Tolerance in vivo}

These experiments were performed with three Beagle dogs and three OphthaCoils, which did not contain an active substance. All animals were healthy during the general examination the day before the application. Immediately before the application one dog had slightly reddened conjunctivae and one dog showed slight seromucous discharge from the eye. Introduction of the OphthaCoils (one coil in the left eye of each animal) proceeded without difficulties. During and immediately after the application all animals were calm and did not show signs of discomfort due to the coils.

During examination of the animals after $8 \mathrm{~h}$, which involved lifting of the lower eyelid, one insert partially came out, showing that all manipulations must be executed very carefully. Based on the observations, it was decided to leave the OphthaCoils in place overnight. The next morning, however, we found that all three animals had lost the insert. One of the dogs still 
had slight discharge from the eye and two dogs had slightly reddened conjunctivae of both eyes.

While we do not have a conclusive explanation for the overnight loss of the device, we presume that the coil is pushed out during sleep, when the eyeball is retracted into the orbit and the third eyelid moves into place [24]. A photograph of a dog with its third eyelid is shown in Figure 3.5. In an attempt to verify this explanation, an additional experiment was executed with a dog that had its third eyelids removed because of lacrimal gland hyperplasia of the third eyelid. The animal received an OphthaCoil in the right eye and the next morning the coil was still present in the eye. This experiment supports the hypothesis that the movement of the third eyelid over the eye is the reason for the loss of the coil overnight.

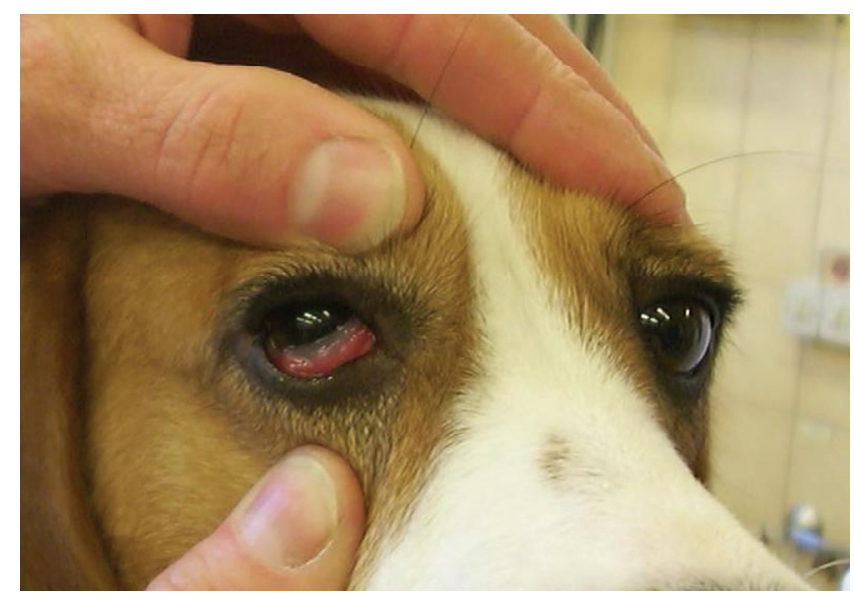

Figure 3.5. Detailed photograph showing the third eyelid. Presumably, the third eyelid pushes the device out of the animal's eye during sleep.

\subsubsection{Release of pradofloxacin in vivo}

These experiments were performed with six Beagle dogs and six OphthaCoils charged with pradofloxacin. The concentration of the anti-infective in the tear fluid was monitored as a function of time. The tear fluid samples were taken with so-called Schirmer test strips. These paper-like porous strips $(5 \times 35 \mathrm{~mm})$ absorb tear fluid when the end of the strip is bent and placed into the lower conjunctival fornix. The procedure is harmless to the animals and, in our experience, the animals were not irritated or disturbed during repeated sampling.

Our experience with the unloaded OphthaCoils (paragraph 3.3.3 vide supra) led us to insert the devices for $2 \times 8 \mathrm{~h}$, under continuous observation of the animals. The OphthaCoils were inserted on the morning of day 1 , taken out after $8 \mathrm{~h}$, and stored at $4^{\circ} \mathrm{C}$. The next day, the OphthaCoils were re-inserted and left in place for another $8 \mathrm{~h}$. Samples were taken at regular 
time points on both days. No major difficulties were encountered, except for dog $\# 6$, which was slightly smaller than the other five animals. Dog \#6 lost the OphthaCoil after $4 \mathrm{~h}$ on the first day. It was decided to re-insert a new OphthaCoil on the second day. However, the new coil was also lost just before the sampling after $8 \mathrm{~h}$. It was clear that this animal had smaller eyes with tighter eyelids, resulting in a non-optimal fit of the device. No problems were encountered with the other animals. They were quiet during the observations, although sometimes a little frightened. We experienced that the sampling procedure had to be executed with great care, as placing of the Schirmer test strip required lifting of the lower eyelid. In some cases, this led to partial escape of the OphthaCoil from the conjunctival fornix. This problem occurred with two animals and could be avoided after some practice.

In view of the apparent tolerance, we decided to leave the OphthaCoil in place at the end of the second day. On the morning of the third day, however, none of the OphthaCoils were still in place. We ascribe this complication to the third eyelid which covers and protects the eyes during sleep. It should be noted that this complication has no immediate implication for extended wear of the OphthaCoil in humans, as humans do not have third eyelids.

Figure 3.6 shows the data which were generated in these experiments. The pradofloxacin concentrations, as measured by re-dissolution of the drug from the Schirmer test strips and analysis by HPLC-MS/MS, are plotted as a function of time. Data from only five dogs are given, because dog \#6 was excluded due to its anatomic characteristics, as mentioned above.

Fast initial release was found, with concentrations in the range $30-70 \mu \mathrm{g} / \mathrm{mL}$ after $1 \mathrm{~h}$. The concentration dropped by two orders of magnitude within the next $7 \mathrm{~h}$. Nevertheless, it is clear that the release continued in the period $8-16 \mathrm{~h}$ (see insert in Figure 3.6). While the concentrations of pradofloxacin on the second day were low, the drug was clearly detectable and its levels were still far above the MIC-values, which are $0.015-0.03 \mu \mathrm{g} / \mathrm{mL}$ for $E$. coli ATCC 8739 and 0.03-0.06 $\mu \mathrm{g} / \mathrm{mL}$ for Staphylococcus aureus ATCC 6538 [23]. But it should be noted that pradofloxacin has an exceptionally high antimicrobial activity. 


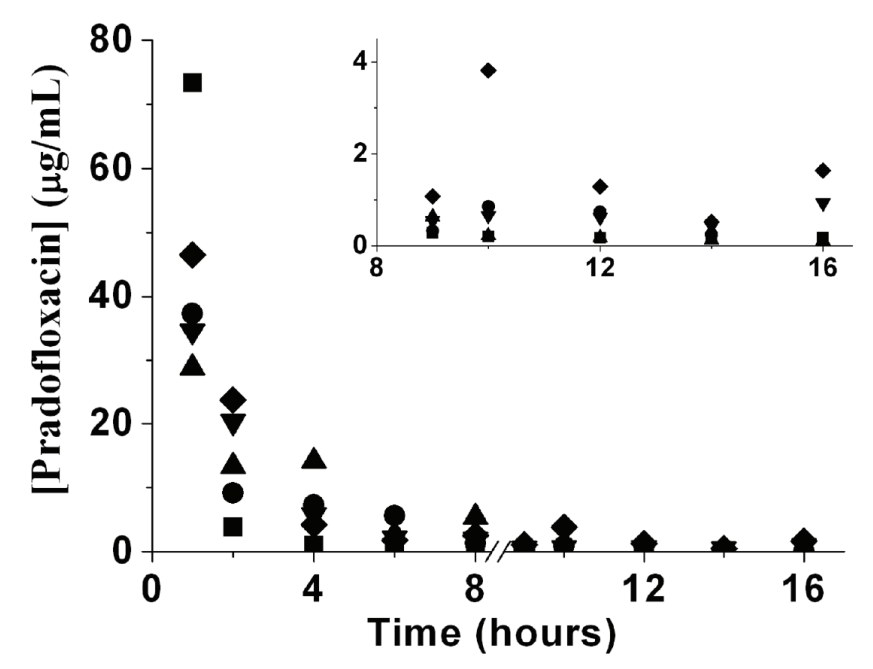

Figure 3.6. Release of pradofloxacin in vivo. As in the in vitro experiments, most of the drug is released in the beginning of the experiment. Concentrations after $16 \mathrm{~h}$, albeit low (see inset), are still far above the MIC-value of pradofloxacin. Note that the interruption of the experiment after $8 \mathrm{~h}$ is indicated on the $x$ axis and the different symbols refer to the five different animals that participated in this experiment.

\subsection{Concluding remarks}

Based on the current data, we believe that the OphthaCoil holds promise as a platform for sustained release of drugs to the eye. A particular advantage of the device may be that it has a stable, flexible, rod-shaped geometry. Already in 1977, Katz and Blackman suggested that rodshaped ocular inserts, having a relatively high aspect ratio (that is, the ratio of length : width), are better tolerated in the conjunctival sac as compared to tablet-shaped inserts, which have a relatively low aspect ratio $[10,25]$. It must be noted that the thin, coiled metallic wire plays an important role in providing sufficient stability to the device. Without the metallic wire the hydrogel would lack sufficient strength and durability, i.e. the device could then fall apart in situ. Furthermore, the OphthaCoil is readily visible in the conjunctival fornix, and its removal using forceps was relatively easy.

However, much more developmental work is necessary. Three major questions should be specifically addressed:

- What is the optimal geometry and flexibility for the coil to ensure that use of the device leads to maximum safety and minimum discomfort? 
- How can the lumen of the coil be used to maximize the capacity of the device?

- What are the most important parameters controlling the drug release kinetics? These parameters should be optimized to achieve the desired in vivo release profile for each particular drug.

Further research and development work aimed at answering these questions is currently in progress in our laboratories.

\section{References}

1. $\quad$ Peppas N.A., Bures P., Leobandung W., and Ichikawa H. Hydrogels in pharmaceutical formulations. Eur J Pharm Biopharm 50 (1) (2000) 27-46.

2. Gupta P., Vermani K., and Garg S. Hydrogels: from controlled release to pH-responsive drug delivery. Drug Discov Today 7 (2002) 569-579.

3. Hoffman A.S. Hydrogels for biomedical applications. Adv Drug Deliv Rev 54 (1) (2002) 3-12.

4. Ratner B.D., Hoffman A.S., Schoen F.J., and Lemons J.E. Biomaterials Science: an introduction to materials in medicine, Elsevier Academic Press, San Diego, California (2004).

5. Li J., Ni X., and Leong K.W. Injectable drug-delivery systems based on supramolecular hydrogels formed by poly(ethylene oxide)s and alpha-cyclodextrin. J Biomed Mater Res 65A (2) (2003) 196202.

6. Coveillo T., Grassi M., Lapasin R., Marino A., and Alhaique F. Scleroglucan/borax: characterization of a novel hydrogel system suitable for drug delivery. Biomaterials 24 (16) (2003) 2789-2798.

7. Lee Y.C., Millard J., Negvesky G.J., Butrus S.I., and Yalkowsky S.H. Formulation and in vivo evaluation of ocular insert containing phenylephrine and tropicamide. Int J Pharm 182 (1) (1999) 121-126.

8. Hornof M., Weyenberg W., Ludwig A., and Bernkop-Schnurch A. Mucoadhesive ocular insert based on thiolated poly(acrylic acid): development and in vivo evaluation in humans. J Control Release 89 (3) (2003) 419-428.

9. $\quad$ Aiache J.M., el Meski S., Serpin G., Beyssac E., and Conti R. Local tolerance and in vivo release study of a new ocular drug delivery system. Drug Deliv Systems and Sciences 1 (3) (2001) 85-87.

10. Saettone M.F., Salminen L. Ocular inserts for topical delivery. Adv Drug Deliv Rev 16 (1995) 95106.

11. Ceulemans J., Vermeire A., Adriaens E., Remon J.P., and Ludwig A. Evaluation of a mucoadhesive tablet for ocular use. J Control Release 77 (3) (2001) 333-344.

12. Sasaki H., Nagano T., Sakanaka K., Kawakami S., Nishida K., Nakamura J., Ichikawa N., Iwashita J., Nakamura T., and Nakashima M. One-side-coated insert as a unique ophthalmic drug delivery system. J Control Release 92 (3) (2003) 241-247.

13. Chetoni P., Di Colo G., Grandi M., Morelli M., Saettone M.F., and Darougar S. Silicone rubber/hydrogel composite ophthalmic inserts: preparation and preliminary in vitro/in vivo evaluation. Eur J Pharm Biopharm 46 (1) (1998) 125-132.

14. Karatas A., and Baykara T. Studies on indomethacin inserts prepared by water-soluble polymers II. The relation between dissolution rate and swelling behaviour. Farmaco 56 (3) (2001) 197-202.

15. Weyenberg W., Vermeire A., Remon J.P., and Ludwig A. Characterization and in vivo evaluation of ocular bioadhesive minitablets compressed at different forces. J Control Release 89 (2) (2003) 329340.

16. Peerlings C.C., Hanssen H.H., Bevers R.T., Boelen E.J., Stelt B.J., Korthagen E.J., and Koole L.H. Heparin release from slippery-when-wet guide wires for intravascular use. J Biomed Mater Res 63 (6) (2002) 692-698. 
17. Hanssen H.J., and Koole L.H. Heparin-releasing intravascular guidewires. Med Device Technol 13 (7) (2002) 20-22.

18. Hanssen H.H., Wetzels G.M., Benzina A., van der Veen F.H., Lindhout T., and Koole L.H. Metallic wires with an adherent lubricious and blood-compatible polymeric coating and their use in the manufacture of novel slippery-when-wet guide wires: possible applications related to controlled local drug delivery. J Biomed Mater Res 48 (6) (1999) 820-828.

19. Pijls R.T., Hanssen H.H., Nuijts R.M., and Koole L.H. Flexible coils with a drug-releasing hydrophilic coating: A new platform for controlled delivery of drugs to the eye? Bio-Med Mater Eng 14 (4) (2004) 383-393.

20. Cruysberg L.P., Nuijts R.M., Geroski D.H., Koole L.H., Hendrikse F., and Edelhauser H.F. In vitro human scleral permeability of fluorescein, dexamethasone-fluorescein, methotrexate-fluorescein and rhodamine $6 \mathrm{G}$ and the use of a coated coil as a new drug delivery system. J Ocul Pharmacol Ther 18 (6) (2002) 559-569.

21. Levinson W., and Jawetz E. Medical microbiology and immunology: examination and board review, Lange Medical Books, New York (1998)

22. Krebber R. Analytical method for the determination of pradofloxacin in serum and urine by turbulent flow chromatography/tandem mass spectrometry. J Vet Pharmacol Therap 26 (1) (2003) 102-103.

23. Wetzstein H.G. Comparative mutant prevention concentrations of pradofloxacin and other veterinary fluoroquinolones indicate differing potentials in preventing selection of resistance. Antimicrob Agents Chemother 49 (10) (2005) 4166-4173.

24. Martin C.L. Ophthalmologische Pharmakologie, in: Augenkrankheiten bei Hund und Katze, Martin, C. L., (Ed.), M\&H Schaper, Alfeld-Hannover (1995).

25. Katz I.M., and Blackman W.M. A soluble sustained-release ophthalmic delivery unit. Am J Ophthalmol 83 (5) (1977) 728-734. 


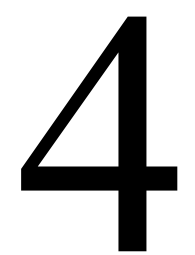

Preliminary study on the patient compliance of the OphthaCoil

Rachel T. Pijls, Lars P.J. Cruysberg, Hans H.L. Hanssen, Rudy M.M.A. Nuijts, and Leo H. Koole. Adapted from: International Journal of Pharmaceutics 341 (2007) 152-161 


\section{Abstract}

To investigate the patient compliance of a new ocular insert, a flexible hydrogel-coated coil was placed in the conjunctival fornix, as part of a study towards a new device for sustained topical delivery of drugs to the anterior segment. The insert without a drug was placed in the conjunctival fornix of the right eye of five healthy volunteers for a $2 \mathrm{~h}$-period. Ocular tolerance was evaluated by comparison of the capillary veins prior to insertion vs. after removal, as well as ophthalmologic examinations and questionnaires. The ease of insertion and removal was also evaluated. Four volunteers responded that the insertion and removal of the insert was not unpleasant. The other, who had an Oriental lid crease, judged the insertion as "a little unpleasant". Three subjects responded that they felt the insert during wear; two experienced a slight tendency to rub their eye. No itching or reddening was observed. None of the volunteers had an increased tear production. The results indicate that the human eye tolerates the coil in the conjunctival fornix, albeit the majority of the subjects (3/5) felt the insert during the $2 \mathrm{~h}$ wear period. These results are similar to sensations reported by first-day wearers of contact lenses. 


\subsection{Introduction}

At least $90 \%$ of all ocular drugs are administered via eye drops, although it has been reported that less than $5 \%$ of the applied drug reaches the intraocular tissues [1-5]. It has been estimated that the average residence time of drug molecules in the tear film is as short as $2-5 \mathrm{~min}$. Therefore, several attempts have been made to improve the corneal penetration of the drug. Ocular inserts and nano/microparticles show significant promise in terms of effective, controlled, and localized drug release [6,7].

Recently, we have introduced a novel ocular insert, see Figure 4.1 [8]. The device is a flexible coil with a drug bearing hydrophilic polymer coating. This coating swells upon placing the coil in the conjunctival fornix, and concomitant release of the drug occurs. Furthermore, the coil's lumen is utilized as an additional reservoir, as described earlier [9]. Drug release from this insert is, hence, a two-step process: burst release from the surface coating is followed by release of drug molecules from the coils lumen. The drug molecules escape from the lumen through diffusion between adjacent windings.

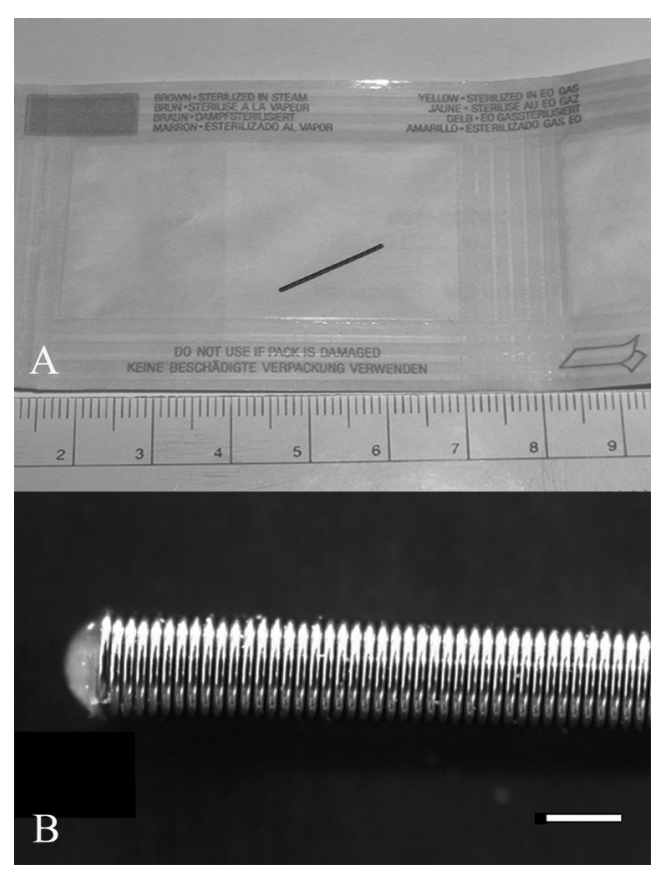

Figure 4.1A. The ocular insert, packed and sterilized. B. Close-up of an end of the device. Polymeric caps effectively prevent sharp edges (bar $=0.5 \mathrm{~mm})$. 
Our insert offers the significant advantage that it retains its mechanical integrity during swelling, i.e. the insert does not leave any hydrogel particles as described by Saettone et al. [3]. This new insert was tested extensively in vitro and in vivo $[8,9]$. The latter experiments were conducted with Beagle dogs. The implanted coils presented no discomfort to the dogs. Experiments with inserts that were charged with pradofloxacin (a fluoroquinolone developed by Bayer HealthCare AG) revealed typical first order decay of the drug concentration in the dog's tear fluid. The concentration remained above the MIC-value for at least $16 \mathrm{~h}$.

Here we describe initial experiments aimed at determining the compliance of the coil in the human eye. Five healthy volunteers (subjects) were enrolled in this pilot study. An insert was placed in the conjunctival fornix of the right eye of each volunteer. These inserts were not charged with any drug, and were left in place for 2 hours. Tolerance was evaluated on the basis of photographs of the capillary blood vessels before and after installation, a qualitative assessment of irritation by an ophthalmologist and subsequent questionnaires filled out by the subjects. The ease of installation and removal of the device was also observed.

\subsection{Materials and methods}

Inserts were manufactured as described previously [10]. The hydrophilic coating on the wire of the coil was a copolymer of $\mathrm{N}$-vinyl pyrrolidone and butyl methacrylate in the molar ratio 70:30. No drug was incorporated in the coating. The dimensions of the coated coils were: length $=16 \mathrm{~mm}$, diameter $=0.6 \mathrm{~mm}$. The ends of each coil were closed with polymeric caps to avoid sharp edges. Three thin wires were present in the lumen of each coil, as described previously $[8,9]$.

The Ethical Committee of the Academic Hospital Maastricht approved the study. Five healthy volunteers (age: 24 - 57 years) gave informed consent prior to the start of the study. Compliance was assessed in three ways: (i), qualitative assessment of irritation by an ophthalmologist; (ii), comparison of the diameters of the capillary veins on photographs taken before installation, and after removal of the device; possible irritation would lead to dilatation of the veins, and (iii), answers to questionnaires, filled out by the subjects.

As a control, the ophthalmologist examined each subject to exclude any eye disease or infection. This qualitative assessment consisted of: best corrected visual acuity, a slit-lamp investigation to exclude any pre-existent anterior surface disease, grading of conjunctival redness $(\mathrm{I}=$ no redness, $\mathrm{V}=$ extensive redness), tear break-up time, Schirmer tear test, fluorescein pattern of the tear film, corneal punctuate staining (diffuse, linear, or none), 
appearance of the anterior chamber, and photography of the ocular surface. All photographs were taken with the same magnification.

After the control examination, the coil was inserted in the conjunctival fornix of the right eye, using forceps. The appearance of the eye, ease of application, and duration of the insertion procedure were observed. Two hours after installation, the next examination was done. The insert was removed and photographs were taken.

Finally, four hours after the removal of the insert, each subject had the last examination, including photographs. The subjects filled out a questionnaire at every time point, answering the questions according to a grading system with numbers scaling from 1 to $5(1=$ not at all, 5 = yes) (see Table 4.1).

\subsection{Results}

Ophthalmologic investigations. Slit-lamp examinations showed a normal appearance of the ocular surface in all volunteers after the inserts were removed. It was noted that subject \#1 had a rather narrow fornix, as he had an Oriental lid crease. Prior to the experiment, redness was grade II for subjects \#1 and \#4, and grade I (no redness) for the rest. No changes in redness were observed throughout and after the experiment. All subjects had normal fluorescein patterns of the tear film, as well as a normal appearance of the anterior chamber, before installation of the insert and after removal. None of the subjects showed corneal punctuate staining before installation of the insert, or afterwards.

Photographic evaluation. Figure 4.2 shows typical photographs that were taken during this study. The left photograph is the right eye of subject \#5 prior to the experiment, the right photograph shows the same eye, immediately after removal of the insert, that had been in the conjunctival fornix for 2 hours. The photographic images of all subjects were shown to be superimposable. Magnifications of the images were performed to determine whether subtle variations in capillary diameter occurred. In all cases, there was no detectable change of the capillary diameter, which indicates that the eyes were not irritated by the insert. The results of the photographic evaluations were consistent for all subjects throughout the experiment. 


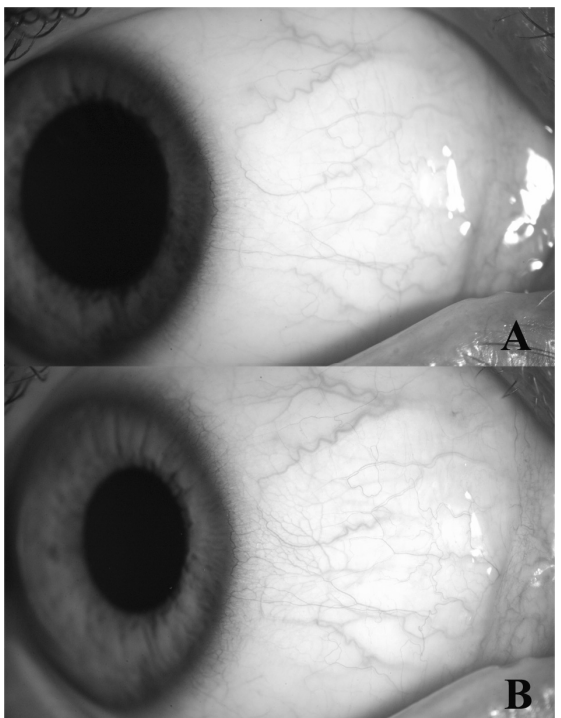

Figure 4.2A. Photograph of the capillary blood vessels of the right eye of a subject before insertion of the device. B. Photograph of the vessels of the same subject directly after removal of the device.

Table 4.1. Results of the questionnaires filled in by the five subjects. The answers were scored by a grading system $(1=$ not at all, $5=$ yes $)$.

\begin{tabular}{lcccccc}
\hline Question & \multicolumn{3}{c}{ Subject \# } & & & \\
& 1 & 2 & 3 & 4 & 5 & \\
\hline During wear of the insert & & & & & & \\
Was the insertion unpleasant? & 4 & 2 & 1 & 1 & 1 & 1.8 \\
Do you feel the insert? & 4 & 2 & 5 & 4 & 1 & 3.2 \\
Do you have a tendency to rub your eye? & 1 & 1 & 1 & 3 & 4 & 2.0 \\
\hline After removal of the insert & & & & & & \\
Was the removal procedure unpleasant? & 3 & 1 & 1 & 1 & 1 & 1.4 \\
Did you feel the insert in the eye? & 4 & 2 & 1 & 4 & 4 & 3.0 \\
Was it unpleasant to wear the insert? & 4 & 1 & 1 & 2 & 3 & 2.2 \\
Did you have a tendency to rub your eye? & 1 & 1 & 1 & 2 & 4 & 1.8 \\
Did your eye produce more tears? & 1 & 1 & 1 & 1 & 1 & 1.0 \\
Did your eye itch? & 1 & 1 & 1 & 1 & 1 & 1.0 \\
Did your eye have a burned feeling? & 1 & 1 & 1 & 1 & 1 & 1.0 \\
Did your eye turn red? & 1 & 1 & 1 & 1 & 1 & 1.0 \\
\hline
\end{tabular}


Questionnaires. Table 4.1 compiles the questions that were asked to the subjects, as well as their answers. The question "Do you feel the insert in the eye?" had the highest score (3.2 during wear, and 3.0 after removal of the insert). This reveals that most of the subjects do feel the presence of the insert in the fornix. In most cases, the feeling is characterized as "a little". Subject \#5 experienced that he had a slight tendency to rub his right eye during wear on the insert. Subject \#1 experienced the installation as "a little unpleasant." Presumably, this relates to the fact that his fornix was relatively narrow due to an Oriental lid crease.

\subsection{Discussion}

The responses to installation, $2 \mathrm{~h}$-wear and removal of the insert are reminiscent of responses that are seen routinely with new wearers of contact lenses (particularly hard contact lenses). These new wearers usually feel the lens shortly after its installation, and they may also experience a tendency to rub the eye. If this parallel is valid, it would imply that patients can get accustomed to wearing the drug delivery insert, perhaps via a special training protocol.

In our opinion, the results of this preliminary study are encouraging, especially with respect to potential short-term applications (i.e., drug delivery to the eye over several hours). Currently, we foresee two such applications:

- Treatment of aggressive corneal infections, such as conjunctivitis and keratitis. The incidence of these infections is increasing, which may attribute, in part, to the growing popularity of contact lenses. In some cases, the infection has to be treated by frequent installation of eye drops that contain an antibiotic

- Controlled delivery of drugs to the eye, prior to and after cataract operations. Before cataract operations, it is mandatory that several drugs are administered to the eye. These include an anaesthetic, antibiotic and a mydriatic agent. Normally the agents are administered sequentially through eye drops. An insert could be used to deliver multiple drugs either sequentially or simultaneously.

Clinical pilot studies into these two directions are currently in preparation. Moreover, further work will concentrate on characterization and optimization of long-term compliance of our device. If sustained release over longer periods (e.g., several days or 1 week) can be realized, then the insert may ultimately prove useful in treatment of uveitis or glaucoma. Moreover, it might provide a better basis to administer ocular drugs to patients who are allergic to the 
preservative agents that are normally present in eye drop solutions as well as providing a treatment that demands less nursing time.

\subsection{Acknowledgements}

This project was carried out through the generous support of DSM Research, Geleen, The Netherlands.

\section{References}

1. Gulsen D., and Chauhan A. Ophthalmic drug delivery through contact lenses. Invest Ophthalmol Vis Sci 45 (7) (2004) 2342-2347.

2. Gulsen D., and Chauhan A. Dispersion of microemulsion drops in HEMA hydrogel: a potential ophthalmic drug delivery vehicle. Int J Pharm 292 (1-2) (2005) 95-117.

3. $\quad$ Saettone M.F., Salminen L. Ocular inserts for topical delivery. Adv Drug Deliv Rev 16 (1995) 95106.

4. Le Bourlais C., Acar L., Zia H., Sado P.A., Needham T., and Leverge R. Ophthalmic drug delivery systems - recent advances. Prog Retin Eye Res 17 (1) (1998) 33-58.

5. $\quad$ Dinslage S., Diestelhorst M., Weichselbaum A., and Suverkrup R. Lyophilisates for drug delivery in ophthalmology: pharmacokinetics of fluorescein in the human anterior segment. Br J Ophthalmol 86 (10) (2002) 1114-1117.

6. $\quad$ Barbu E., Sarvaiya I., Green K.L., Nevell T.G., and Tsibouklis J. Vinylpyrrolidone-co-(meth)acrylic acid inserts for ocular drug delivery: Synthesis and evaluation. J Biomed Mater Res A 74 (4) (2005) 598-606.

7. Kaur I.P., and Kanwar M. Ocular preparations: the formulation approach. Drug Dev Ind Pharm 28 (5) (2002) 473-493.

8. Pijls R.T., Hanssen H.H., Nuijts R.M., and Koole L.H. Flexible coils with a drug-releasing hydrophilic coating: A new platform for controlled delivery of drugs to the eye? Bio-Med Mater Eng 14 (4) (2004) 383-393.

9. Pijls R.T., Sonderkamp T., Daube G.W., Krebber R., Hanssen H.H., Nuijts R.M., and Koole L.H. Studies on a new device for drug delivery to the eye. Eur J Pharm Biopharm 59 (2) (2005) 283-288.

10. Hanssen H.H., Wetzels G.M., Benzina A., van der Veen F.H., Lindhout T., and Koole L.H. Metallic wires with an adherent lubricious and blood-compatible polymeric coating and their use in the manufacture of novel slippery-when-wet guide wires: possible applications related to controlled local drug delivery. J Biomed Mater Res 48 (6) (1999) 820-828. 


\section{Increasing the capacity of the OphthaCoil using drug-loaded microspheres}

Rachel T. Pijls, Aylvin A. Dias, and Leo H. Koole Adapted from: International Journal of Pharmaceutics 341 (2007) 152-161

The work described in this chapter also led to the filing of a patent: 'Coiled wire for the controlled release of drugs to the eye' WO2007/006427 (Appendix I) 


\section{Abstract}

To increase the capacity of the OphthaCoil, without affecting the device's flexibility, drugloaded microspheres were introduced in the inner lumen of the device. Poly(2-hydroxyethyl methacrylate)- and poly(2-hydroxyethyl methacrylate-co-1-vinyl-2-pyrrolidone)-microspheres were prepared by suspension polymerization. The resultant particles were swollen in a highly concentrated solution of either the dye fluorescein sodium or the antibiotic chloramphenicol and lyophilized. The loaded particles were introduced in the central cavity of the ocular device. In vitro release profiles showed a 6-fold increase of the capacity for the dye fluorescein sodium, but not for the antibiotic chloramphenicol. Flexibility measurements revealed that by introducing microspheres in the central cavity of the device, the flexibility did not decrease. 


\subsection{Introduction}

Ocular drugs are normally administered via eye drops. Although this is common practice, there are several problems and drawbacks, such as: (i) limited bioavailability, (ii) rapid elimination, (iii) destabilization of the tear film, (iv) non compliance by patients, and (v) allergic reactions [1-12]. These practical issues have stimulated the search for alternative methods for ocular drug delivery. Much of this work was devoted to ocular inserts, which serve as a platform for the release of one or more active substances. It has become clear, however, that the development of an ocular insert that reliably combines controlled release with absence of any irritation to the patient, poses a formidable technical challenge [13-15].

Recently, we have proposed an ocular drug delivery device according to a new concept [16, 17]. The device, called OphthaCoil (Figure 5.1), consists of a drug loaded adherent hydrogel on a thin metallic wire, which is coiled. The coiled structure accounts for the device's flexibility and integrity. The ends of the coil are sealed with caps using photo-curable cyanoacrylate glue and the device is gas-sterilized before use. The device is placed in the conjunctival fornix. The hydrogel coating starts to swell once the device contacts the tear fluid, and concomitant release of the drug into the tear film occurs.

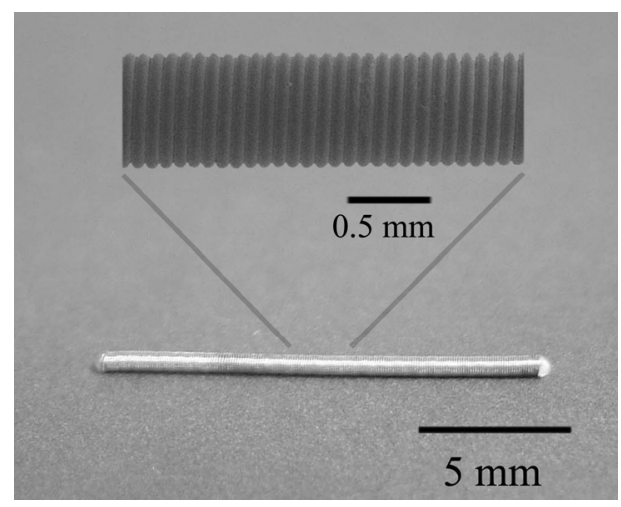

Figure 5.1. The ocular drug delivery device

Previously, we have reported that the OphthaCoil is well tolerated in animal models, notably in the eyes of Beagle dogs [17]. Moreover, we have shown that it is possible to release antibiotics into the tear film, in such a way that the concentration remains above the minimal inhibitory concentration (MIC-value) for 16 hours at least.

In this study, we describe further improvement of the OphthaCoil, especially in terms of drug capacity and patient comfort. Two ideas were explored: $(i)$, to use the interior of the coil as an additional drug reservoir; this implies that drug molecules must be introduced into the coil's 
cavity, and that these molecules must pass the windings of the coil in the release process; (ii), to use polymeric microspheres as drug carriers. Microspheres were used since filling the interior with pure drug (as a powder) is not practical; the use of a concentrated aqueous solution of the drug to fill the lumen is impossible since this would lead to premature swelling of the hydrogel coating; and the use of drug loaded hydrogel coated filaments (as described in our previous work [17]) would translate in an increased stiffness of the device.

The hypothesis is that the use of drug-loaded polymeric microspheres offers the possibility to load the interior cavity of the coil, without compromising its flexibility. The microspheres can move independently in the cavity when the coil is bent, and thus maintain the flexibility of the device when compared to the use of wire filaments.

The agents used for loading of the microspheres are the dye fluorescein sodium and the antibiotic ciprofloxacin. The chemical structures of both agents are given in Figure 5.2. Ciprofloxacin is a fluoroquinolone antibacterial agent, which is widely used in human and veterinary medicine [18]. Ciprofloxacin is used to treat bacterial infections of the eye, such as conjunctivitis or keratitis [19-21]. It is also prescribed to treat corneal ulcers, when the delicate layer covering the surface of the eye is ulcerated [22]. Fluoroquinolones are bactericidal, exert their effect by DNA gyrase inhibition, and have a broad spectrum of activity against both Gram negative and Gram positive bacteria [23, 24].

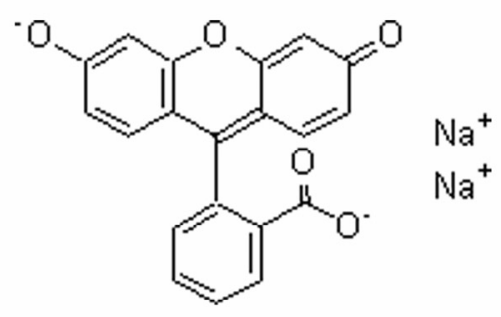

A Fluorescein sodium

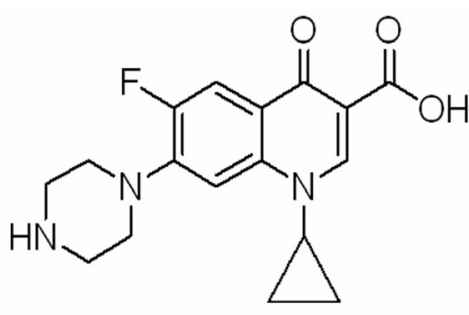

B Ciprofloxacin

Figure 5.2. The chemical structures of fluorescein sodium (A) and chloramphenicol (B)

Here, new data on the release of the dye and the antibiotic from three different OphthaCoil prototypes will be reported. The first series has the dye/drug exclusively in the hydrogel coating of the coil; the second series has the dye/drug exclusively in the interior (that is within the hydrogel microspheres); and the third has the dye/drug both in the hydrogel coating and in the interior of the coil. Furthermore, the structural stiffness of prototypes filled on three different ways are measured (a coil with an empty interior, a coil with three hydrogel coated filaments and a coil with the hydrogel microspheres in the interior). 


\subsection{Materials and Methods}

\subsubsection{Preparation of the ocular device}

All chemicals were purchased from Acros Organics (Geel, Belgium), unless stated otherwise. The hydrogel coating Slipskin ${ }^{\circledR}$ was prepared from N-vinyl pyrrolidone (NVP, Aldrich, Steinheim, Germany) and butyl methacrylate (BMA) as described earlier [25]. A NVP:BMA molar ratio of 70:30 was used. The co-polymer was dissolved in N-methyl pyrrolidone (NMP) at $10 \% \mathrm{w} / \mathrm{w}$ under continuous mechanical stirring for $24 \mathrm{~h}$. The procedure to apply the copolymer onto a thin metallic wire was described earlier [26-28]. Briefly, a stainless steel wire (thickness of $76 \mu \mathrm{m}$ ) was pulled through the copolymer solution and guided through a cylindrical oven (maximal temperature $300^{\circ} \mathrm{C}$, height approximately $6 \mathrm{~m}$ ). This resulted in the evaporation of the solvent NMP and residual free monomers. In our case, first a $1 \mu \mathrm{m}$ layer of poly(ethersulfone) (PES) metal primer coating was applied followed by a 4-5 $\mu \mathrm{m}$ layer of the co-polymer. This process leads to a dual coating on the metal wire with uniform thickness. In order to coil the wire, a steel core wire with a diameter of $432 \mu \mathrm{m}$ was connected to a speedcontrollable electromotor, and the coated wires were wound around the core wire. Coils of approximately $50 \mathrm{~cm}$ length were obtained. Then, the core wire was removed and the coil was cut into pieces of $15 \mathrm{~mm}$ in length. The ends of each coil were sealed with a small cap consisting of UV-cured poly(cyanoacrylate), which is a suitable sealing and bonding agent [29, 30]. These caps serve two purposes: (i) they prevent that any sharp edges (due to cutting of the wire) could damage or irritate the cornea of tissues inside the fornix, and (ii) they close the space inside the coil, i.e. transport of drug from the lumen of the coil to the tear fluid is limited to diffusion along a pathway in between adjacent windings of the coil. Note that the placement of the caps was done stepwise: after placement of the first cap, we first filled the lumen with (drug-loaded) microspheres. Then, the second cap was placed.

To create a drug delivery device, the hydrogel coating of the wire could be incorporated with a drug. The drug was dissolved in the NMP, together with the $70 / 30 \% \mathrm{~mol} / \mathrm{mol} \mathrm{NVP} / \mathrm{BMA}$ copolymer. Then, the coating procedure was performed as described above to apply the drug loaded coating composition onto the wire.

\subsubsection{Preparation of the microspheres}

The monomers 2-hydroxyethyl methacrylate (HEMA) and NVP were used as the principal building blocks for the microspheres. Poly(HEMA) is a well-known material in biomedical applications, because of its non-toxicity, non-irritability and biocompatibility. Examples of its applications are soft contact lenses [31-33], blood-contacting materials and artificial emboli in 
endovascular embolization [34, 35]. Poly(NVP) is used because of its hydrophilicity and swelling properties. It is present in many eye drop formulations [36], and is also known to have excellent biocompatibility when implanted in the vitreous body or used as a vitreous substitute [37].

The microspheres were prepared by suspension polymerization of the appropriate monomer in a non-solvent using suitable stabilizing agents to prevent the agglomeration of the monomer droplets during polymerization [34, 35]. In the case of highly water soluble monomers like HEMA, the dispersion of the monomer into droplets has to be carried out in concentrated salt solutions in which the solubility of the monomer is very low [38]. One type of microspheres consisted of the monomer HEMA solely, named poly(HEMA)-spheres and the other type consisted of both the monomers HEMA and NVP in a 70/30-weight ratio, named poly(HEMAco-NVP)-spheres.

In the preparation of the poly(HEMA)-spheres, the polymerization of the monomer HEMA was done in an aqueous medium containing $17 \mathrm{w} \%$ sodium chloride and $0.672 \mathrm{w} \%$ magnesium hydroxide as described by Jayakrishnan [34, 35]. A $250 \mathrm{~mL}$ three-neck round-bottomed flask, fitted with a half-moon stirrer was charged with a solution containing $14.45 \mathrm{~g}$ sodium chloride and $1.97 \mathrm{~g}$ magnesium chloride hexahydrate in $70 \mathrm{~mL}$ water. The flask was heated to $70^{\circ} \mathrm{C}$ in a thermostatic oil bath and $0.783 \mathrm{~g}$ sodium hydroxide was added in $15 \mathrm{~mL}$ water with continuous stirring to precipitate the suspension stabilizer magnesium hydroxide. The temperature was raised to $80^{\circ} \mathrm{C}$ and the dispersed phase (total weight of $36 \mathrm{~g}$ ) was introduced drop wise into the flask. The dispersed phase contained the monomer HEMA (96.8 w\%), the cross-linker tetraethyleneglycol dimethacrylate (tetra-EGDMA) (3.0 w\%, Fluka Chemie, Steinheim, Germany) and the initiator $\alpha, \alpha^{\prime}$-azoisobutyronitrile (AIBN) $(0.2 \mathrm{w} \%)$. The suspension was stirred at 150 rpm for 4 hours. After cooling down the suspension, the stabilizer magnesium hydroxide was dissolved by adding dilute hydrochloric acid (Merck, Germany). The beads were washed several times with distilled water and dried under reduced pressure at $35^{\circ} \mathrm{C}$.

The preparation of the poly(HEMA-co-NVP)-spheres $(70 / 30 \mathrm{w} \%)$ was similar to the preparation of poly(HEMA)-spheres mentioned above. The only difference was that the dispersed phase, contained the monomers HEMA (67.8 w\%) and NVP (29.0 w\%), the cross linker tetra-EGDMA $(3.0 \mathrm{w} \%)$ and the initiator AIBN $(0.2 \mathrm{w} \%)$. Also this batch was dried under reduced pressure at $35^{\circ} \mathrm{C}$.

\subsubsection{Characterization and drug loading of the microspheres}

The prepared poly(HEMA)- and poly(HEMA-co-NVP)-spheres were characterized by size, shape and swelling. The size distribution was measured using standard test sieves (Retsch, 
Germany). The beads were sieved into different size-fractions using standard test sieves of 200, 300 and $425 \mu \mathrm{m}$. The shape and surface of the microspheres were investigated with scanning electron microscopy. The average size $(n \geq 200)$ and swelling $(n \geq 30)$ of the microspheres was determined with photographs taken with an optical microscope (Nikon Eclipse 800). The photographs were characterized with the program ImageJ (version 1.32j).

To measure the swelling of both types of microspheres, a dry microsphere (size range 200-300 $\mu \mathrm{m})$ was put under the light microscope and a picture was taken. Then, the microspheres were swollen in an excess of water for $24 \mathrm{~h}$ at room temperature. The equilibrium swelling ratio $\left(\mathrm{Q}_{\mathrm{v}}\right)$ was calculated by the formula:

$$
\mathrm{Q}_{\mathrm{v}}=\mathrm{D} / \mathrm{D}_{\mathrm{o}}
$$

with $\mathrm{D}$ and $\mathrm{D}_{\mathrm{o}}$ the swollen and initial (dry) diameters of the microspheres respectively $[39,40]$. To investigate whether the swelling medium will influence the equilibrium swelling ratio $\mathrm{Q}_{v}$, the microspheres were also measured in simulated tear fluid (STF) [41] and ethanol. The agents used for loading of the microspheres are the dye, fluorescein sodium and the antibiotic, ciprofloxacin. Four different microspheres according to Table 5.1 were used for the in vitro release experiments. In order to load the microspheres with fluorescein sodium, a concentrated solution of fluorescein sodium of $75 \mathrm{mM}$ was prepared in demineralized water. One gram of poly(HEMA)-spheres was placed in a test sieve (diameter of $25 \mathrm{~mm}$, pore size $100 \mu \mathrm{m}$ ), after which the sieve was placed in the fluorescein-solution. After 8 hours, the sieve was removed from the solution and the microspheres were lyophilized.

Table 5.1. The microspheres used for the in vitro release experiments

\begin{tabular}{ccc}
\hline Microsphere & Material & Drug \\
\hline I & Poly(HEMA) & Fluorescein sodium \\
II & Poly(HEMA-co-NVP) & Fluorescein sodium \\
III & Poly(HEMA) & Ciprofloxacin \\
IV & Poly(HEMA-co-NVP) & Ciprofloxacin \\
\hline
\end{tabular}

Then, a dispersion of $12 \mathrm{mM}$ ciprofloxacin (Fluka Chemie, Steinheim, Germany) in ethanol was made. This dispersion was less concentrated than the fluorescein sodium solution because of solubility limits of ciprofloxacin in water and ethanol. One gram of poly(HEMA)-spheres was placed in a test sieve, which was subsequently placed in the dispersion for $8 \mathrm{~h}$. The drug loaded microspheres were then lyophilized. The same procedure was followed to load the poly(HEMA-co-NVP)-spheres with fluorescein sodium and ciprofloxacin respectively. 


\subsubsection{Drug release}

The drug-loaded microspheres were introduced into the inner lumen of the coil with a miniature funnel. The ends were sealed with the photo-curable cyanoacrylate cap and drug release was measured at room temperature in the following set-up. STF was perfused through a silicon tube (inner diameter of $1 \mathrm{~mm}$ ) at a rate of $100 \mu \mathrm{L} / \mathrm{min}$. A coil was placed at the end of the silicone tube and fractions of approximately $150 \mu \mathrm{L}$ were collected.

Table 5.2. The microsphere loaded coils used for the in vitro release experiments

\begin{tabular}{ccc}
\hline Coil & Coating coil & Microspheres \\
\hline A & Fluorescein sodium & None \\
B & None & I \\
C & Fluorescein sodium & I \\
D & None & II \\
E & None & III \\
F & None & IV \\
\hline
\end{tabular}

Several microsphere loaded coils were measured to investigate the contribution of the microspheres to the capacity and the release time of the device. Table 5.2 shows the coils used for the release experiments. First the contribution of drug loaded microspheres in the central cavity of the coil was investigated by comparing six types of coils:

- a coil with a fluorescein sodium loaded coating and an empty cavity (coil A); the hydrogel coating of the fluorescein sodium loaded coils contained $9 \mathrm{w} \%$ of the dye (i.e. $43 \mu \mathrm{g}$ per coil),

- a blank coil (with no active ingredient in the hydrogel coating) with fluorescein sodium loaded poly(HEMA)-spheres in the inner cavity (coil B); the microspheres released $33 \mu \mathrm{g}$ fluorescein sodium (vide infra),

- the combination: fluorescein sodium loaded hydrogel coating on the coil and fluorescein sodium loaded poly(HEMA)-spheres in the inner cavity of the coil (coil C); the coils contained $43+33=76 \mu \mathrm{g}$ fluorescein sodium,

- a blank coil with fluorescein sodium loaded poly(HEMA-co-NVP)-spheres in the cavity (coil D); the microspheres released $70 \mu \mathrm{g}$ fluorescein sodium (vide infra),

- a blank coil with ciprofloxacin loaded poly(HEMA)-spheres in the inner cavity (coil E); coil E released $1 \mu \mathrm{g}$ of ciprofloxacin (vide infra), 
- a blank coil with ciprofloxacin loaded poly(HEMA-co-NVP)-spheres in the inner cavity (coil F); coil F released $6 \mu \mathrm{g}$ (vide infra).

The constant flow through the tube of $100 \mu \mathrm{L} / \mathrm{min}$, allows the amount of drug released to be calculated by the determination of the area under the curve (AUC) of the release curve (concentration plotted against time). The concentrations of fluorescein sodium in the fractions collected were measured directly after sampling with a spectrofluorometer (SpectraMax Gemini, containing the SoftMax Pro software) with the absorption and emission wavelengths $\lambda_{\mathrm{abs}}=490 \mathrm{~nm}$ and $\lambda_{\mathrm{em}}=514 \mathrm{~nm}$ respectively [42].

The concentrations of ciprofloxacin in the collected fractions were evaluated using a zone of inhibition assay within a month after the release measurement. The samples were stored at $18^{\circ} \mathrm{C}$ until the bio-assay. Plastic petridishes $(\mathrm{d}=14 \mathrm{~cm})$ were filled level with $60 \mathrm{~mL}$ Isosensitest ${ }^{\mathrm{TM}}$ medium. After coagulation and drying, the indicator bacterium E. coli ATCC 25922 was brought onto the plates. Seven holes $(r=4 \mathrm{~mm})$ were punched at equal distance in the agar of every petridish. Finally, these holes were filled with $100 \mu \mathrm{L}$ of the collected fractions or the dilution series. After 24 hours of incubation at $37^{\circ} \mathrm{C}$ under atmospheric conditions, semi-confluent growth appeared on the total agar surface and the diameters of the inhibition zones were measured. The concentrations of ciprofloxacin in the collected fractions could be inferred from a dilution series (concentrations in the range of $0.02-8.0 \mu \mathrm{g} / \mathrm{mL}$ ).

\subsubsection{Flexibility tests}

Flexibility tests were done with different coils to determine whether filling the central cavity with microspheres compromises the flexibility of the coils. A three-point bending test was performed on: (i) a coil with an empty cavity, (ii) a coil with three hydrogel coated filaments inside, (iii) a coil with microspheres in the inner cavity.

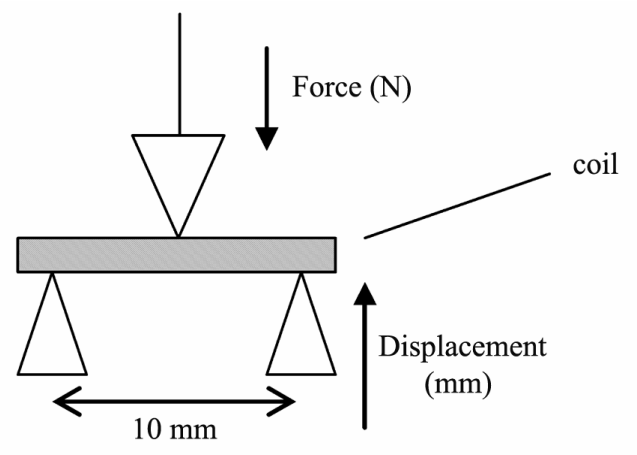

Figure 5.3. Schematic set up three-point bending test for the coil (flexibility test) 
The flexibility of the coils was measured with a rheometric solids analyser (RSA3), equipped with RSI-Orchestrator V6.5 software. The ends of each coil were placed onto two solid points and a force was applied to the centre of the coil as shown in Figure 5.3. The displacement was set at $2.5 \mathrm{~mm}$ and the force as a function of the displacement was measured at the centre of the coil. All coils were measured in the dry and wet state (both $n=4$ ), to investigate whether wetting of the coils influences the bending forces.

\subsection{Results and Discussion}

\subsubsection{Preparation of the coils}

Preparation of all coils was successful. Coils with the Slipskin ${ }^{\circledR}$ coating were prepared, as well as coils with $9 \mathrm{w} \%$ of fluorescein sodium and $91 \mathrm{w} \%$ Slipskin ${ }^{\circledR}$ in the coating. Inspection of the outer surface of the coated wires and coils (by optical microscopy and scanning electron microscopy) showed perfectly smooth surfaces. Coiling of the coated wires did not lead to any damage of the coating (i.e. there were no visible cracks). Earlier tests showed that the coating of the coil has no residual solvents or monomers after the coating procedure [26].

\subsubsection{Preparation and characterization of the microspheres}

Two different series of microspheres were prepared successfully: cross-linked poly(HEMA)and cross-linked poly(HEMA-co-NVP)-spheres, in which the mass ratio HEMA:NVP was 70:30. The cross-linker was tetra-EGDMA ( $3 \%$ by mass) in all cases. The yields of the poly(HEMA) microspheres was $27.2 \mathrm{~g}$ (75\%; theoretically expected $36.0 \mathrm{~g}$ ), and the yield of the poly(HEMA-co-NVP) microspheres was $20.0 \mathrm{~g}$ (95\%; theoretically expected $21.2 \mathrm{~g}$ ). All microspheres were thoroughly washed with demineralized water, to remove possible leachable substances (oligomers and unreacted monomers).

The average size diameters of the poly(HEMA)- and poly(HEMA-co-NVP)-spheres (both $\mathrm{n} \geq$ $300)$ were $195( \pm 80.0) \mu \mathrm{m}$ and $290( \pm 98.2) \mu \mathrm{m}$ respectively. Further data on the size distribution are given in Table 5.3. The desired fraction, diameters between 200 and $300 \mu \mathrm{m}$, was selected through sieving. Microspheres in this size range can easily be inserted in the interior of the coil, and there remains sufficient space to allow them to swell. Figure 5.4 shows scanning electron microscopy (SEM) photographs of the sieved microspheres, and reveals that both types of microspheres were spherical and had a smooth surface.

Table 5.4 compiles the data on equilibrium swelling of the microspheres in water, STF, and ethanol. There was no significant difference in swelling of the poly(HEMA)-spheres and the 
poly(HEMA-co-NVP)-spheres in both water and STF. However, swelling of both types of microspheres in ethanol resulted in significantly different swelling ratios compared to their swelling in water or STF $(\mathrm{p}<0.05)$.

Table 5.3. Size distribution of the microsphere batches

\begin{tabular}{ccc}
\hline Size $(\mu \mathrm{m})$ & Poly(HEMA) batch & Poly(HEMA-co-NVP) batch \\
\hline$<200$ & $19.6 \mathrm{w} \%$ & $5.3 \mathrm{w} \%$ \\
$200-300$ & $17.8 \mathrm{w} \%$ & $22.4 \mathrm{w} \%$ \\
$300-425$ & $28.4 \mathrm{w} \%$ & $25.2 \mathrm{w} \%$ \\
$>425$ & $36.4 \mathrm{w} \%$ & $47.1 \mathrm{w} \%$ \\
\hline
\end{tabular}

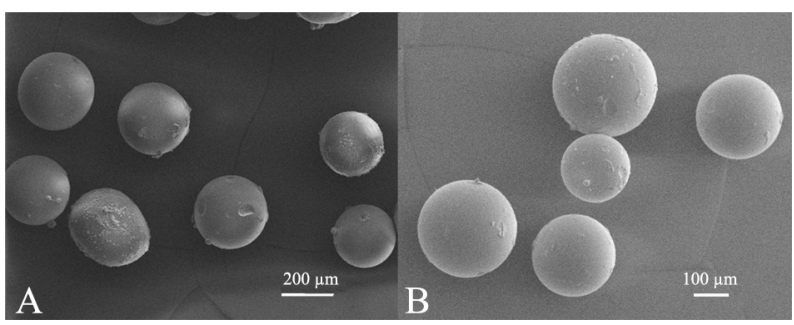

Figure 5.4. SEM-photographs of the the microspheres $(A=\operatorname{poly}(H E M A), B=\operatorname{poly}(H E M A-c o-N V P))$

Table 5.4. Equilibrium swelling ratio (Qv) of the microspheres in the swelling media $(n \geq 30)$

\begin{tabular}{ccc} 
& $\mathrm{Qv}_{\text {(polyHEMA) }}(\mathrm{SD})$ & $\mathrm{Qv}_{\text {(polyHEMA-co-NVP) }}(\mathrm{SD})$ \\
\hline Water & $1.166(0.031)$ & $1.234(0.015)$ \\
STF & $1.176(0.019)$ & $1.256(0.033)$ \\
Ethanol & $1.492(0.054)$ & $1.409(0.037)$ \\
\hline
\end{tabular}

\subsubsection{Drug release}

Figure 5.5 displays the release profiles measured for the coils A $-\mathrm{C}$, which were loaded with fluorescein sodium, either exclusively in the hydrogel coating of the coil (coil A), or exclusively in the coil's lumen (coil B), or both in the hydrogel coating and the interior (coil C). Note that the release curves were measured in four-fold.

In line with previous observations, coil A releases most of the dye in the first hour; measured concentrations in the effluent SLF drop from $7.4 \mu \mathrm{g} / \mathrm{mL}$ to appr. $0.05 \mu \mathrm{g} / \mathrm{mL}$ at $\mathrm{t}=60 \mathrm{~min}$. Coil B shows virtually no initial release; a maximum concentration of appr. $2.2 \mu \mathrm{g} / \mathrm{mL}$ is reached after $80 \mathrm{~min}$. During the next $4 \mathrm{~h}$, the concentration gradually drops to approximately $0.3 \mu \mathrm{g} / \mathrm{mL}$. The release curve measured for coil $\mathrm{C}$ combines the features of coils A and B: 
fluorescein release from coil C declines from appr. $7.2 \mu \mathrm{g} / \mathrm{mL}$ initially, to appr. $0.3 \mu \mathrm{g} / \mathrm{mL}$ after $5 \mathrm{~h}$.

From integration of the release curves A and B, it can be calculated that approximately $19 \mu \mathrm{g}$ of fluorescein sodium was released from coil A and $33 \mu \mathrm{g}$ from coil B. Integration of the release curve $\mathrm{C}$ leads to a total release of approximately $123 \mu \mathrm{g}$. Hence, it can be concluded that the device's capacity has increased by appr. $500 \%$ by using dye loaded microspheres compared to a coil with an empty interior.

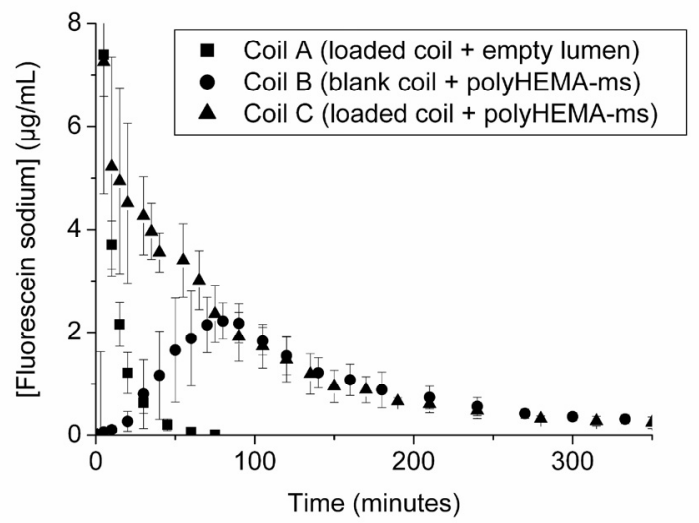

Figure 5.5. Contribution of the microspheres in the central cavity of the coil to the release profile

Figure 5.6 shows a comparison of the performance of the poly(HEMA)-spheres versus the poly(HEMA-co-NVP)-spheres $(n=4)$. The release curves of fluorescein sodium in Figure 5.6A show that poly(HEMA-co-NVP)-spheres are a much more effective carrier than the poly(HEMA)-spheres. Integration of the curves led to $33 \mu \mathrm{g}$ of fluorescein sodium released for coil B (poly(HEMA)-spheres) vs. $70 \mu \mathrm{g}$ of fluorescein sodium released for coil D (poly(HEMA-co-NVP)-spheres). This difference can be explained, at least in part, by the fact that poly(HEMA) swells much less than poly(HEMA-co-NVP) under our experimental conditions. The data reveal that the loading percentages are $2.4 \%$ (SD 0.41) for the poly(HEMA)-spheres and 5.4\% (SD 0.65) for the poly(HEMA-co-NVP)-spheres. Note that we cannot determine the entrapment efficiency, since the maximum capacity of each of the materials for fluorescein sodium is not known.

The curves in Figure 5.6B show the data measured for release of ciprofloxacin under the same conditions. Two conclusions can be drawn directly: (i) much less ciprofloxacin, as compared to fluorescein sodium, is absorbed in both cases (i.e. 1.0 and $6.0 \mu \mathrm{g}$ for poly(HEMA)- and poly(HEMA-co-NVP)-spheres respectively). The loading percentages are 0.084\% (SD 0.027) for ciprofloxacin in poly(HEMA)-spheres and $0.367 \%$ (SD 0.087) for ciprofloxacin in 
poly(HEMA-co-NVP)-spheres; (ii) poly(HEMA-co-NVP) is a more efficient absorber of ciprofloxacin compared to poly(HEMA). The latter observation may be explained by a favourable matrix-host interaction, probably hydrogen bonding between the $\mathrm{C}=\mathrm{O}$ groups of the NVP-links in the co-polymer, and a proton-donating site of ciprofloxacin.
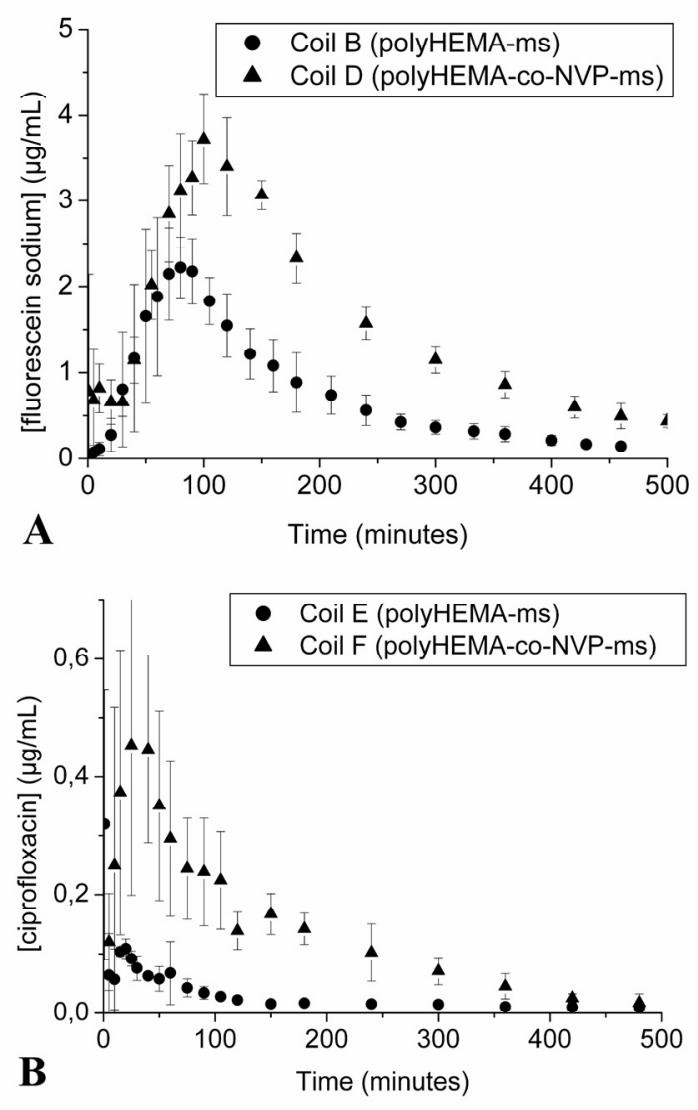

Figure 5.6. Release curves of a blank coil filled with all four types of microspheres $(A=$ fluorescein sodium, $B=$ ciprofloxacin)

Closer inspection of the release curves in Figures 5.6A and 5.6B reveals another difference: release of fluorescein sodium is negligible during the first 20 minutes. Then, the concentration of the dye rises, passes a maximum and decreases again. This pattern implies that diffusion of the drug across the device's wall is the rate-determining step in the release mechanism. On the other hand, the release curves for ciprofloxacin show an initial maximum and continuous decrease afterwards. This suggests that, for ciprofloxacin, the release from the microspheres is the rate-determining step; the drug easily passes through the windings of the coil. 
Obviously, the amount of ciprofloxacin on the microspheres is only marginal. Apparently, the drug loading of microspheres is very dependent on the solubility of the drug and the swelling medium.

\subsubsection{Flexibility test}

Flexibility of the coil is an important factor that can affect the comfort of this ocular device and acknowledging that the conjunctival space of the eye has a curvature. The flexibility of several coils was determined with three-point bending tests. The coils used had an empty central cavity, hydrogel coated filaments in the inner cavity or microspheres in the lumen. In Figure 5.7, the forces are plotted against the displacement of the coil. It can be seen that the coils with an empty cavity and with microspheres in the cavity are more flexible, i.e. less force is necessary for the displacement, than the coils with the hydrogel coated filaments in the inner cavity. The coils with microspheres in the central cavity had a stiffness approximately two times higher than the empty coils (this measured with the displacement set at $2.5 \mathrm{~mm}$ out of a length of $10 \mathrm{~mm}$ ). The coils with the three coated filaments in the central cavity had much higher values for the force/displacement than both other types of coils. Wetting the coils did not have a statistically significant effect on the stiffness.

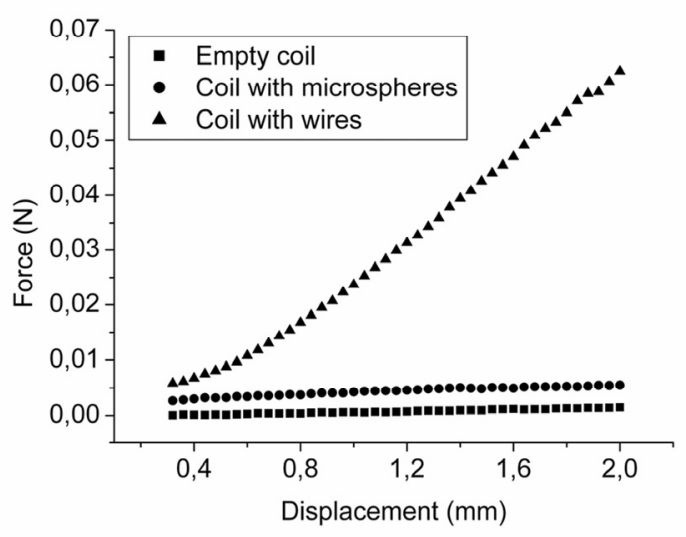

Figure 5.7. The flexibility experiment with three different coils

\subsection{Concluding remarks}

The idea to use drug loaded polymeric microspheres to increase the capacity of the OphthaCoil, without affecting the flexibility of the coiled structure, appears to be technical feasible. Using fluorescein sodium as a drug-mimic, it was found that a $500 \%$ increase of the 
released compound could be realized. By introducing dye or drug loaded microspheres in the lumen of the coil not only the capacity can be increased, but also the time window of the dye released can be increased from 1 hour to over 5 hours. Note that the drug release in vitro is probably faster than in vivo, due to a difference in the flow; i.e. in the in vitro set up the flow was set at $100 \mu \mathrm{L} / \mathrm{min}$, whereas in vivo the tear turnover rate is only $0.5-2.2 \mu \mathrm{L} / \mathrm{min}[43,44]$.

Yet, the total amount of fluorescein sodium released from the OphthaCoil remains low. For example, the total release of fluorescein sodium from coil $\mathrm{C}$ (that contained the dye in both the surface coating and in the lumen) was $123 \mu \mathrm{g}$. For comparison: a $0.5 \%$ eye drop with a volume of 20-30 $\mu \mathrm{L}$ contains 100-150 $\mu \mathrm{g}$ of drug. As mentioned earlier, the bioavailability of an eye drop is less than $5 \%$. This means that only 5-7.5 $\mu \mathrm{g}$ of an eye drop will reach the intraocular tissues. Evidently, the crucial point is the bioavailability of drugs released from the OphthaCoil. Our previous work indicated this to be significantly larger than in the case of eye drops. If the bioavailability of drugs from our insert would be $10 \%$, than the charge of coil C corresponds to 2 eye drops. However, with increasing bioavailability, the amount of drug absorbed is also increased. For example, a coil with a drug bioavailability of $50 \%$ can be compared to 8-12 eye drops. It can be concluded that it is essential to achieve maximum drug loading of the microspheres.

The way of loading the microspheres with a drug by soaking them into a concentrated solution is especially useful for hydrophilic drugs. The uptake of drugs from a concentrated solution is suitable for relatively rapid release. However, also hydrophobic drugs can be incorporated into the microspheres by the synthesis of the microspheres in the presence of the hydrophobic drug, rather than charging them afterwards through swelling. This will result in relatively slower release. This was not done here, because we focus on relatively rapid release (1-12 hours).

In our opinion, the results of the preliminary patient study are encouraging, especially with respect to potential short-term applications (i.e., drug delivery to the eye over several hours). Currently, we foresee two such applications:

(i) Treatment of aggressive corneal infections (i.e. corneal ulcers) and severe bacterial conjunctivitis and fungal keratitis. The incidence of these infections is increasing, partly due to (soft) contact lens wear. There is evidence, that contact lens use has surpassed trauma as the most common risk factor for fungal keratitis. The percentage of fungal ulcers caused by nontherapeutic contact lenses doubled from 25\% between 1999 and 2001, to approximately $45 \%$ in 2005 and 2006 [45]. Fusarium and candida are common causes of fungal keratitis. According to Iyer, most patients can be treated successfully with dual antifungal therapy: natamycin (5\%) and amphotericin B $(0.15 \%)$ are the most commonly used drugs [44]. In some cases, the infection has to be treated by frequent installation of eye drops that contain an antibiotic. To 
treat corneal ulcers, patients have to apply, day and night, ciprofloxacin eye drops every fifteen minutes for the first six hours, every thirty minutes for the next eighteen hours. On day 2 , patients have to administer the drops every 2 hours and for the next 3-14 days, every four hours [22]. Evidently this treatment puts a heavy burden on patients and health care workers, as the patients will be hospitalized during the first days and this ultimately has impact on health care cost. We expect that delivery of antibiotics from our device can alleviate this burden. Depending on the potency of the antibiotic agent, we calculated that sustained delivery over a minimum of 5 hours could be realized. This implies that the two-day therapy can be realized with a maximum of 10 device exchanges for the first 48 hours of treatment, which compares favorably with $>70$ drop-installations.

(ii) Controlled delivery of drugs to the eye, prior to cataract surgery. Before cataract operations, it is mandatory that several drugs are administered to the eye. These include an anaesthetic, antibiotic and several mydriatic agents. Normally these agents are administered sequentially through eye drops. A device could be used to deliver multiple drugs either sequentially or simultaneously. The time scale of release required is of the same order of the coil release characteristics, since the health care workers administer antibiotics and mydriatica to patients approximately $2 \mathrm{~h}$ prior to surgery.

A clinical pilot study into the second application is currently in preparation. Moreover, further work will concentrate on characterization and optimization of long-term compliance of our coil. If sustained release over longer periods (e.g., several days or 1 week) can be realized, then the coil may ultimately prove useful in treatment of uveitis or glaucoma. It might provide a better basis to administer ocular drugs to patients who are allergic to the preservative agents that are normally present in eye drop solutions as well as providing a treatment that demands less time of health care workers.

\subsection{Acknowledgements}

The authors would like to thank MCTec BV (Venlo, the Netherlands) for the preparation of the coated coils and EPflex (Dettingen, Germany) for sealing the ends of the coils. The assistance from R. v.d. Heijden (University of Maastricht, the Netherlands) with the preparation and characterization of the microspheres, M. Hazen (CPV, Maastricht, the Netherlands) with the zone of inhibition assays and R. van Sluijs (DSM Research, Geleen, the Netherlands) with the mechanical flexibility experiments is gratefully acknowledged. 


\section{References}

1. Winfield A.J., Jessiman D., Williams A., and Esakowitz L. A study of the causes of non-compliance by patients prescribed eyedrops. Br J Ophthalmol 74 (8) (1990) 477-480.

2. Van Ooteghem M. Properties influencing drug retention, in: Biopharmaceutics of ocular drug delivery, Edman, P., (Ed.), CRC Press, Inc., Boca Raton, Florida (1993) 29-33.

3. Lang J.C. Ocular drug delivery conventional ocular formulations. Adv Drug Deliv Rev 16 (1995) 3943.

4. Le Bourlais C., Acar L., Zia H., Sado P.A., Needham T., and Leverge R. Ophthalmic drug delivery systems - recent advances. Prog Retin Eye Res 17 (1) (1998) 33-58.

5. Oyster C.W. The human eye: structure and function, Sinauer Associates, Inc., Sunderland, Massachusetts (1999).

6. Geroski D.H., and Edelhauser H.F. Drug delivery for posterior segment eye disease. Invest Ophthalmol Vis Sci 41 (5) (2000) 961-964.

7. Van Santvliet L., and Ludwig A. Determinants of eye drop size. Surv Ophthalmol 49 (2) (2004) 197213.

8. Baudouin C. Allergic reaction to topical eyedrops. Curr Opin Allergy Clin Immunol 5 (5) (2005) 459463.

9. Gulsen D., and Chauhan A. Dispersion of microemulsion drops in HEMA hydrogel: a potential ophthalmic drug delivery vehicle. Int J Pharm 292 (1-2) (2005) 95-117.

10. Hosoya K., Lee V.H., and Kim K.J. Roles of the conjunctiva in ocular drug delivery: a review of conjunctival transport mechanisms and their regulation. Eur J Pharm Biopharm 60 (2) (2005) 227-240.

11. Salyani A., and Birt C. Evaluation of an eye drop guide to aid self-administration by patients experienced with topical use of glaucoma medication. Can J Ophthalmol 40 (2) (2005) 170-174.

12. Barbu E., Verestiuc L., Nevell T.G., and Tsibouklis J. Polymeric materials for ophthalmic drug delivery: trends and perspectives. J Mater Chem 16 (34) (2006) 3439-3443.

13. Ding S. Recent developments in ophthalmic drug delivery. Pharm Sci Technol Today 1 (8) (1998) 328-335.

14. Herrero-Vanrell R., and Refojo M.F. Biodegradable microspheres for vitreoretinal drug delivery. Adv Drug Deliv Rev 52 (1) (2001) 5-16.

15. Barbu E., Sarvaiya I., Green K.L., Nevell T.G., and Tsibouklis J. Vinylpyrrolidone-co-(meth)acrylic acid inserts for ocular drug delivery: Synthesis and evaluation. J Biomed Mater Res A 74 (4) (2005) 598-606.

16. Pijls R.T., Hanssen H.H., Nuijts R.M., and Koole L.H. Flexible coils with a drug-releasing hydrophilic coating: A new platform for controlled delivery of drugs to the eye? Bio-Med Mater Eng 14 (4) (2004) 383-393.

17. Pijls R.T., Sonderkamp T., Daube G.W., Krebber R., Hanssen H.H., Nuijts R.M., and Koole L.H. Studies on a new device for drug delivery to the eye. Eur J Pharm Biopharm 59 (2) (2005) 283-288.

18. Rosemary M.J., Suryanarayanan V., Ganapati Reddy P., Maclaren I., Baskaran S., and Pradeep T. Ciprofloxacin@ $\mathrm{SiO}_{2}$ : Fluorescein nanobubbles. Proc. Indian Acad. Sci. (Chem. Sci.) 115 (5-6) (2003) 703-709.

19. Parks D.J., Abrams D.A., Sarfarazi F.A., and Katz H.R. Comparison of topical ciprofloxacin to conventional antibiotic therapy in the treatment of ulcerative keratitis. Am J Ophthalmol 115 (4) (1993) 471-477.

20. Wilhelmus K.R., Hyndiuk R.A., Caldwell D.R., Abshire R.L., Folkens A.T., and Godio L.B. 0.3\% ciprofloxacin ophthalmic ointment in the treatment of bacterial keratitis. The Ciprofloxacin Ointment/Bacterial Keratitis Study Group. Arch Ophthalmol 111 (9) (1993) 1210-1218.

21. Hyndiuk R.A., Eiferman R.A., Caldwell D.R., Rosenwasser G.O., Santos C.I., Katz H.R., Badrinath S.S., Reddy M.K., Adenis J.P., and Klauss V. Comparison of ciprofloxacin ophthalmic solution $0.3 \%$ to fortified tobramycin-cefazolin in treating bacterial corneal ulcers. Ciprofloxacin Bacterial Keratitis Study Group. Ophthalmology 103 (11) (1996) 1854-1863.

22. Health Information Patient UK. http://www.patient.co.uk (2006). 
23. Ke T.L., Cagle G., Schlech B., Lorenzetti O.J., and Mattern J. Ocular bioavailability of ciprofloxacin in sustained release formulations. J Ocul Pharmacol Ther 17 (6) (2001) 555-563.

24. Schaefer F., Bruttin O., Zografos L., and Guex-Crosier Y. Bacterial keratitis: a prospective clinical and microbiological study. Br J Ophthalmol 85 (7) (2001) 842-847.

25. Aldenhoff Y.B., Knetsch M.L., Hanssen J.H., Lindhout T., Wielders S.J., and Koole L.H. Coils and tubes releasing heparin. Studies on a new vascular graft prototype. Biomaterials 25 (16) (2004) 31253133.

26. Hanssen H.H., Wetzels G.M., Benzina A., van der Veen F.H., Lindhout T., and Koole L.H. Metallic wires with an adherent lubricious and blood-compatible polymeric coating and their use in the manufacture of novel slippery-when-wet guide wires: possible applications related to controlled local drug delivery. J Biomed Mater Res 48 (6) (1999) 820-828.

27. Peerlings C.C., Hanssen H.H., Bevers R.T., Boelen E.J., Stelt B.J., Korthagen E.J., and Koole L.H. Heparin release from slippery-when-wet guide wires for intravascular use. J Biomed Mater Res 63 (6) (2002) 692-698.

28. Knetsch M.L., Aldenhoff Y.B., Schraven M., and Koole L.H. Human endothelial cell attachment and proliferation on a novel vascular graft prototype. J Biomed Mater Res 71A (4) (2004) 615-624.

29. Ressemann T.V., Hackett S.S., Keith P.T. Intravascular catheter with distal tip guide wire lumen, US5921958 (1996).

30. Kim Y.M., and Gupta B.K. 2-octyl cyanoacrylate adhesive for conjunctival wound closure in rabbits. J Pediatr Ophthalmol Strabismus 40 (3) (2003) 152-155.

31. Karlgard C.C., Jones L.W., and Moresoli C. Ciprofloxacin interaction with silicon-based and conventional hydrogel contact lenses. Eye Contact Lens 29 (2) (2003) 83-89.

32. Gulsen D., and Chauhan A. Ophthalmic drug delivery through contact lenses. Invest Ophthalmol Vis Sci 45 (7) (2004) 2342-2347.

33. Sato T., Uchida R., Tanigawa H., Uno K., and Murakami A. Application of polymer gels containing side-chain phosphate groups to drug-delivery contact lenses.J Appl Polym Sci 98 (2) (2005) 731-735.

34. Jayakrishnan A., Sunny M.C., and Thanoo B.C. Polymerization of 2-hydroxyethyl methacrylate as large size spherical beads. Polymer 31 (1990(a)) 1339-1342.

35. Jayakrishnan A., and Thanoo B.C. Suspension polymerization of 2-hydroxyethyl methacrylate in the presence of polymeric diluents: a novel route to spherical highly porous beads for biomedical applications. J Biomed Mater Res 24 (7) (1990(b)) 913-927.

36. Gobbels M., and Spitznas M. Corneal epithelial permeability of dry eyes before and after treatment with artificial tears. Ophthalmology 99 (6) (1992) 873-878.

37. Bruining M.J., Edelbroek-Hoogendoorn P.S., Blaauwgeers H.G., Mooy C.M., Hendrikse F.H., and Koole L.H. New biodegradable networks of poly(n-vinyl pyrrolidone) designed for controlled nonburst degradation in the vitreous body. J Biomed Mater Res 47 (2) (1999) 189-197.

38. Jayakrishnan A., Chithambara Thanoo B., Rathinam K., Ravi Mandalam K., Rao V.R.K., Lal A.V., and Mohanty M. Hydrogel microspheres from cross-linked poly(methyl methacrylate): synthesis and biocompatibility studies. Bull Mater Sci 12 (1) (1989) 17-25.

39. Horák D., Metalova M., and Rypácek F. New radiopaque polyHEMA-based hydrogel particles. J Biomed Mater Res 34 (2) (1997) 183-188.

40. Oral E., and Peppas N.A. Hydrophilic molecularly imprinted poly(hydroxyethyl-methacrylate) polymers. J Biomed Mater Res A 78 (1) (2006) 205-210.

41. Paulsson M., and Edsman K. Controlled drug release from gels using surfactant aggregates I: Effect of lipophilic interactions for a series of uncharged substances. J Pharm Sci 90 (9) (2001) 1216-1225.

42. Mota M.C., Carvalho P., Ramalho J., and Leite E. Spectrophotometric analysis of sodium fluorescein aqueous solutions. Determination of molar absorption coefficient. Int Ophthalmol 15 (5) (1991) 321326.

43. Schoenwald R.D. Ocular pharmacokinetics, in: Textbook of ocular pharmacology, Zimmermann T.J., Lippinocott-Raven Publishers, Philadelphia (1997) 119-138.

44. Ghate D., and Edelhauser H.F. Ocular drug delivery. Expert Opin Drug Deliv 3 (2) (2006) 275-287.

45. Iyer S.A. Tuli S.S., and Wagoner R.C. Fungal keratitis: emerging trends and treatment outcomes. Eye Contact Lens 32 (6) (2006) 267-271. 


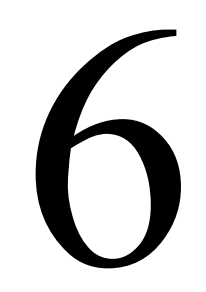

\section{Pradofloxacin release from the OphthaCoil}

Rachel T. Pijls, Sandra Lindemann, Rudy M.M.A. Nuijts, Gert W. Daube, and Leo H. Koole. Adapted from: Journal of Drug Delivery Science and Technology 17 (2007) 87-91 


\section{Abstract}

The release of the anti-infective pradofloxacin from a new ocular insert called the OphthaCoil was evaluated. The ocular insert consists of a drug-loaded adherent hydrogel on a thin wire, which is coiled. The inner lumen of the coil was filled with a polymer rod made from a poly(2hydroxyethyl methacrylate) hydrogel and loaded with the same drug. The in vitro release of pradofloxacin and its pharmacokinetics in canine tear fluid was evaluated over a $72 \mathrm{~h}$ time interval. The results show that in vitro release is faster than in vivo release, probably because the refresh time for tear fluid in the eye is slower than in the in vitro experiments. In vivo, the drug level in the tear fluid remained within the concentration range during the entire $72 \mathrm{~h}$, which is when pradofloxacin is expected to be active against the most relevant bacteria species involved in canine conjunctival infections. These results are quite promising for the future application of the OphthaCoil in veterinary or human ophthalmology. 


\subsection{Introduction}

Installation of eye drops to administer a drug to the tear film is common, but far from ideal. It is well known that over $95 \%$ of the drug is spilled and will not reach the intraocular tissues [13]. Most of the drug is lost almost immediately through blinking and clearance through the nasolacrimal duct. The limited bioavailability of ocular drugs poses significant problems in human ophthalmology, as well as in veterinary medicine. Moreover, it is known that many patients (and pet owners) have problems in following a strict regimen of a series of daily eye drop installations. This situation has prompted numerous attempts to develop new ocular drug delivery systems. New approaches could improve the contact time of an agent with the ocular tissue and/or increase the penetration of the drug into the eye. Ocular inserts and nano/microparticles show significant promise in terms of effective, controlled, and sustained drug release $[4,5]$. These are devices to be inserted on or in the eye (e.g., a drug releasing contact lens, or a depot in the conjunctival fornix) to release the drug in an effective, sustained and controlled manner.

Bacterial ocular infections (e.g. conjunctivitis) are commonly treated with eye drops that contain one or more antibacterial agents. Fluoroquinolones, such as ciprofloxacin and moxifloxacin, are arguably the best class of antibiotic to treat and/or prevent ocular infections [6]. Fluoroquinolones have a broad spectrum of activity; they kill rapidly, and have more than a 10-year history of being non-toxic when used topically. In addition, this class of antimicrobial drugs has the best ocular penetration of any of the commercially available topical antibiotics [6-8].

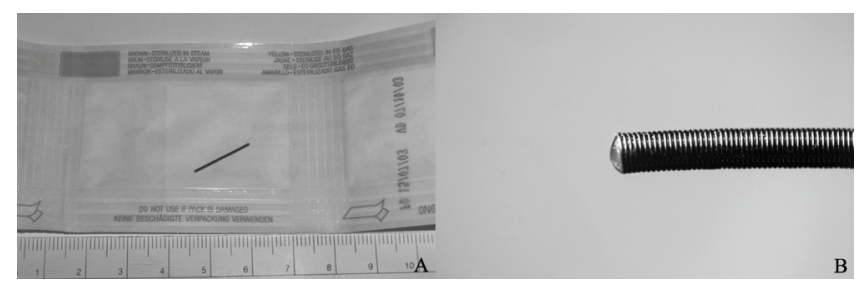

Figure 6.1A. Photograph of the sterilized ocular device. B. Light microscope photograph of a closed end.

Recently, we reported on a conceptually new ocular drug delivery insert: the OphthaCoil [9, 10]. This device consists of a flexible coil with a diameter of approximately $0.6 \mathrm{~mm}$, and a length of $16 \mathrm{~mm}$ (Figure 6.1). It is inserted into the lower conjunctival fornix. The OphthaCoil's exterior has a synthetic hydrogel coating. This coating swells in an aqueous environment, but also adheres to a metallic substrate. The substrate is a thin, coiled, stainless 
steel wire ( $76 \mu \mathrm{m}$ diameter) that provides the coiled structure with both flexibility and stability. The hydrogel coating is impregnated with a drug (e.g. an antibiotic or anti-inflammatory agent), and the release process starts after the coating comes into contact with the tear fluid. The drug capacity of the OphthaCoil can be substantially increased by using the lumen as an additional reservoir. In this way, a two-step drug release mechanism is obtained: fast release from the OphthaCoil's surface, followed by slower release of the drug from the OphthaCoil's interior.

Here, we report on the progress of our work on the OphthaCoil as a device for controlled ocular drug delivery. Specifically, we describe that (i) the OphthaCoil's drug capacity can be increased by a factor 2.5 through insertion of a drug-filled poly(2-hydroxyethyl methacrylate) rod in the coil's lumen, (ii) installation of the OphthaCoil in an animal model (Beagle dogs) did not cause any tolerance problems or other side effects for $72 \mathrm{~h}$, and (iii) release of a potent antiinfective (pradofloxacin) occurred in vitro and in vivo; concentration levels in the tear film in vivo remained above the minimal inhibitory concentration (MIC value) for $72 \mathrm{~h}$.

\subsection{Materials and methods}

\subsubsection{Manufacture of the OphthaCoil}

All chemicals were purchased from Acros Organics, Belgium, unless stated otherwise. The hydrogel coating was prepared from N-vinyl pyrrolidone (NVP, Aldrich, Steinheim, Germany) and butyl methacrylate (BMA). A mixture of 70:30 NVP:BMA (mol:mol) together with 0.044 mol\% $\alpha, \alpha^{\prime}$-azoisobutyronitrile (AIBN) was prepared and transferred into Teflon tubes with an inner diameter of $12 \mathrm{~mm}$. The tubes were immersed in a thermostatic oil bath equipped with a programmable time/temperature control system (PM Lauda, Köningshofen, Germany). The time/temperature profile used was described previously [11].

The polymerization resulted in a hard, opaque material. The copolymer was removed from the tubes and dissolved in N-methyl pyrrolidone (NMP, Merck, Hohenbrunn, Germany) (concentration $10 \%$ by mass) under continuous mechanical stirring. The copolymer was applied as a thin, adherent coating onto a long stainless steel wire (approximately $400 \mathrm{~m}$ in length and $76 \mu \mathrm{m}$ in diameter) according to the extrusion-like procedure that was described previously [12-14].

Briefly, a stainless steel wire was guided through the copolymer solution (at a speed of 80 $\mathrm{m} / \mathrm{min}$ ) and through a cylindrical oven (temperature $300^{\circ} \mathrm{C}$, height approximately $6 \mathrm{~m}$ ). This resulted in the evaporation of the NMP solvent and residual-free monomers and apposition of 
the copolymer as a thin, uniform coating onto the wire's surface. Deposition of the coating was verified with continuous, online measurements of the wire's thickness after coating. In our case, a 1- $\mu \mathrm{m}$ layer of poly(ethersulfone) (PES) was first applied followed by a 4- $\mu \mathrm{m}$ layer of the copolymer. The PES-layer shows excellent adherence to metallic surfaces and acts as a binder for the hydrophilic polymer. The extrusion-like process leads to a cylindrical coating with uniform thickness.

A steel core wire with a diameter of $432 \mu \mathrm{m}$ was connected to a speed-controllable electromotor, and the coated wires were coiled around it. Coils of approximately $50 \mathrm{~cm}$ in length were obtained. Then, the core wire was removed and the coil was cut into pieces of 15 $\mathrm{mm}$ in length. These coils were not loaded with an active ingredient. The ends of the device can be closed with caps. The devices were then sterilized with EtO. Figure 6.1 shows a sterilized device (A) and a close-up of one end of the device with the polymeric cap (B).

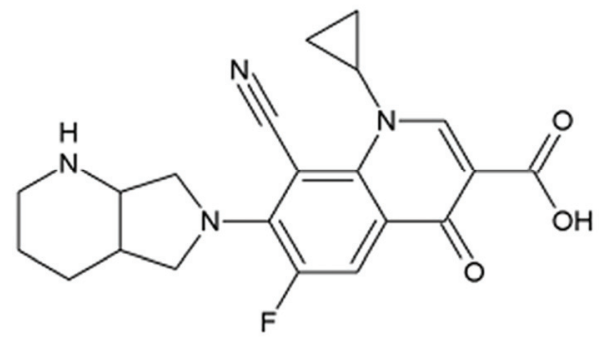

Figure 6.2. Chemical structure of pradofloxacin.

Additionally, a drug can be incorporated in the hydrogel coating of the coiled wire. Here, we will focus on the release of pradofloxacin (Bayer HealthCare AG, Monheim, Germany). The chemical structure of pradofloxacin is given in Figure 6.2. Pradofloxacin, an 8cyanofluoroquinolone, is being developed to treat bacterial infections in dogs and cats [15]. To incorporate the drug into the hydrogel coating, both the drug and the copolymer were dissolved in the NMP before the extrusion-like procedure to apply the coating onto the wire. Coils without the drug in the hydrogel coating (coil A) and coils with $40 \mathrm{w} \%$ pradofloxacin (Batch NK-101203-0) in the hydrogel coating (coil B) were prepared.

The inner lumen of the coils was filled with a polymeric rod made from poly(2-hydroxyethyl methacrylate). PolyHEMA has been used in many devices for controlled drug delivery. The material has excellent chemical and thermal stability and a high level of biocompatibility. PolyHEMA is used widely in ophthalmology as, among other uses, a constituent element of intraocular and soft contact lenses [2, 16-18]. The preparation of the polymeric rods was performed in a water bath at $100^{\circ} \mathrm{C}$. A formulation of $90.5 \mathrm{w} \%$ 2-hydroxyethyl methacrylate 
(HEMA), $4.5 \mathrm{w} \%$ pradofloxacin, $4.0 \mathrm{w} \%$ cross-linker tetra-ethylene glycol dimethacrylate (EGDMA, Fluka, Steinheim, Germany) and $1.0 \mathrm{w} \%$ of an AIBN initiator was prepared. This formulation was injected into a tube (inner diameter of $0.5 \mathrm{~mm}$, outer diameter of $2.5 \mathrm{~mm}$ ) made from silicon rubber. The tube had a length of approximately $40 \mathrm{~cm}$. The tube, filled with the formulation, was placed in the water bath for one hour, after which it was removed from the water bath and cooled down for $30 \mathrm{~min}$. The polyHEMA rod did not stick to the lumen of the tube, and its removal was straightforward. The tube was cut into 13-mm pieces. These pieces were placed in the lumen of the prepared coils. Figure 6.3 shows a schematic crosssection of both types. In summary, device A had a blank hydrogel coated coil (coil A) with a drug-loaded polymeric rod in the lumen, and device B had a drug-loaded hydrogel coating on the coil (coil B) and the drug-loaded polymeric rod in the lumen. Again, the ends of the prepared devices were closed by using caps.
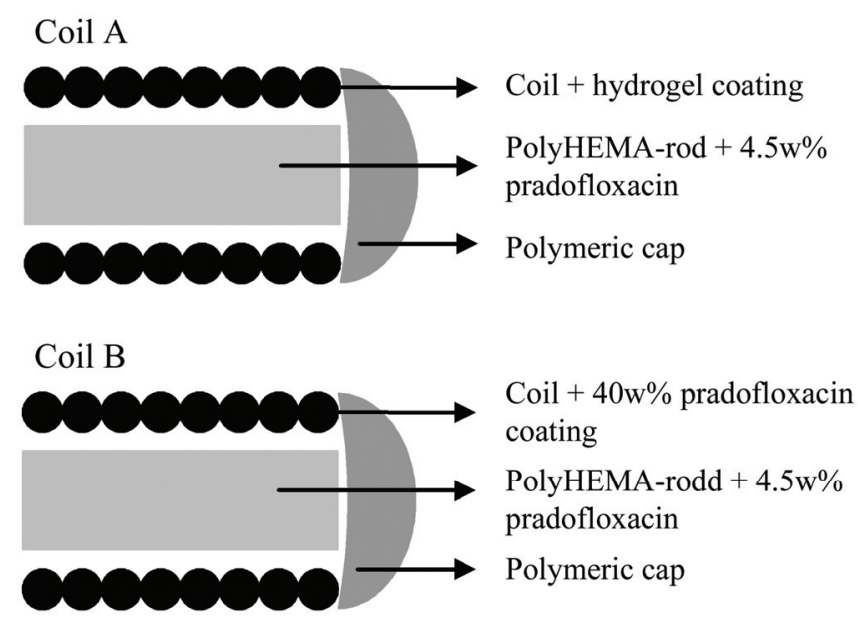

Figure 6.3. A schematic cross-section of the device with the polyHEMA rod in the lumen of the coil.

\subsubsection{In vitro release}

First, the release kinetics of pradofloxacin from the devices were evaluated in vitro using the following procedure. Simulated Tear Fluid [19] was perfused with a Varian 9010 Pump through a tube (inner diameter of $1 \mathrm{~mm}$ ) with a flow of $100 \mu \mathrm{L} / \mathrm{min}$. The device was placed at the distal end of the tube and fractions of approximately $150 \mu \mathrm{L}$ were collected in Eppendorf vials at different intervals. The two types of devices prepared above were measured twice. The fractions were stored at $-18^{\circ} \mathrm{C}$ immediately after being collected.

A bioassay was used to determine drug concentrations in the fractions. This proves that the pradofloxacin was still active after the preparation of the device. The bioassay was performed 
with the indicator bacterium, E. coli ATCC 25922, within a month after the release experiment. A dilution series was made from pradofloxacin in Simulated Tear Fluid. Plastic Petri dishes (diameter of $14 \mathrm{~mm}$ ) were filled to level with $60 \mathrm{~mL}$ of Iso-sensitest medium. After coagulation and drying, the indicator bacterium was placed onto the plate. Seven holes (radius of $4 \mathrm{~mm}$ ) were punched at equal distance in the agar bottom of every Petri dish. Finally, these holes were filled with either $100 \mu \mathrm{L}$ of the collected samples or the dilution series. After $24 \mathrm{~h}$ of incubation at $37^{\circ} \mathrm{C}$ with atmospheric conditions, semi-confluent growth has appeared on the total agar surface and the diameters of the zones of inhibition were measured. The concentration of pradofloxacin could be calculated using the diameters of the samples of the dilution series.

The results of these experiments were compiled in a graph that plots the concentration of pradofloxacin as a function of time. The time axis could be readily converted into a volume axis since the flow rate was constant $(100 \mu \mathrm{L} / \mathrm{min})$ throughout the experiment. The curve of the graph [pradofloxacin] versus elution volume (i.e. determination of the area under the curve (AUC)) was integrated with the Matlab 6.0 program. This integration gave the mass for the amount of pradofloxacin that was released.

\subsubsection{In vivo release}

The pharmacokinetics of pradofloxacin in the tear fluid of Beagle dogs were evaluated. Coils with a pradofloxacin-loaded hydrogel coating and a pradofloxacin-loaded polyHEMA rod in the lumen of the coil were used for this experiment (i.e. device B). Four Beagles were used in the study ( 2 females, 2 males). They were 1.5 to 2 years old, and their body weight ranged between 8 and $12 \mathrm{~kg}$. The dogs underwent an inclusion examination. The exclusion criteria were growing dogs, pregnant or nursing dogs, and animals with a poor state of health and/or symptoms of a serious disease, especially of the ocular system.

Prior to application of the coils, the dogs underwent general intravenous anesthesia with 0.08 $\mathrm{ml} / \mathrm{kg}$ body weight of diazepam $10 \mathrm{mg}$ (Diazep von ct, ct-Arzneimittel, Berlin, Germany), followed by $0.04 \mathrm{~mL} / \mathrm{kg}$ body weight of Domitor (medetomidine hydrochloride, $1 \mathrm{mg} / \mathrm{mL}$, Pfizer, Karlsruhe, Germany). A coil was then inserted into the lower conjunctival fornix of each eye with a pair of tweezers and attached to the conjunctiva with one stitch using Supramid monofil suture material (Heiland, Hamburg, Germany). The coils were attached to the conjunctiva with a single stitch in order to avoid premature loss of the coil, as experienced in previous experiments [10]. Immediately after the coils were placed, medetomidine anesthesia was antagonized with $0.04 \mathrm{~mL} / \mathrm{kg}$ body weight of Antisedan (atipamezole hydrochloride, 5 $\mathrm{mg} / \mathrm{mL}$, Pfizer, Karlsruhe, Germany) injected intramuscularly. 
Lacrimal samples were taken with blank filter paper discs (Antibiotika-Testblättchen, 6-mm diameter, Schleicher \& Schuell, Dassel, Germany). The samples were taken at the following time intervals: before application, $0.5,1,2,4,6,8,24,32,48$ and $72 \mathrm{~h}$ after application. The presence of the coils was verified and documented before each sampling. The discs were inserted into the lower conjunctival sac of each eye with a pair of tweezers and were left there for one minute (Figure 6.4). Afterwards, the discs were placed in prelabeled micro test tubes (Reaktionsgefäss $2 \mathrm{~mL}$, Fa. Eppendorf, Hamburg, Germany). The samples were stored at $18^{\circ} \mathrm{C}$ or below until analytical analysis. After sampling, the coils were removed $72 \mathrm{~h}$ after application by cutting the sutures with scissors and removing the material with a pair of tweezers. The animals were anesthetized as described above.

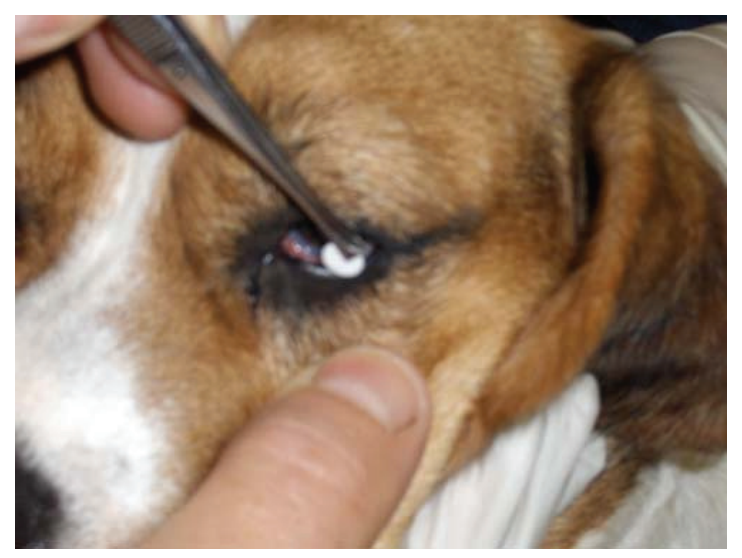

Figure 6.4. Photograph of tear fluid sampling with a blank paper disc.

The samples were analyzed through a zone of inhibition assay, as described in section 6.2.2. A dilution series was made by dissolving pradofloxacin in Simulated Tear Fluid. Blank paper discs were soaked in these solutions and frozen in Eppendorf vials afterwards. During the bioassay, both the sample discs and dilutions series discs were placed on top of the culture plate. All discs were defrosted one hour before the analysis and each Petri dish could contain seven samples. Finally, after 24 hours of incubation at $37^{\circ} \mathrm{C}$ under atmospheric conditions, the diameters of the inhibition zones were measured and the concentrations of pradofloxacin were calculated. In order to exclude false-positive reactions resulting from any possible antibacterial activity from the collection device or anesthetics, a blank disc and the anesthetics used in the study were also examined. 


\subsection{Results and discussion}

\subsubsection{In vitro release}

The release profiles of pradofloxacin, measured twice in the in vitro perfusion system, are given in Figure 6.5. Figure 6.5A refers to the release from device A (i.e. pradofloxacin in the coil's lumen, but not in the surface coating), and Figure 6.5B shows the release from device B (i.e. pradofloxacin in the lumen and in the surface coating).
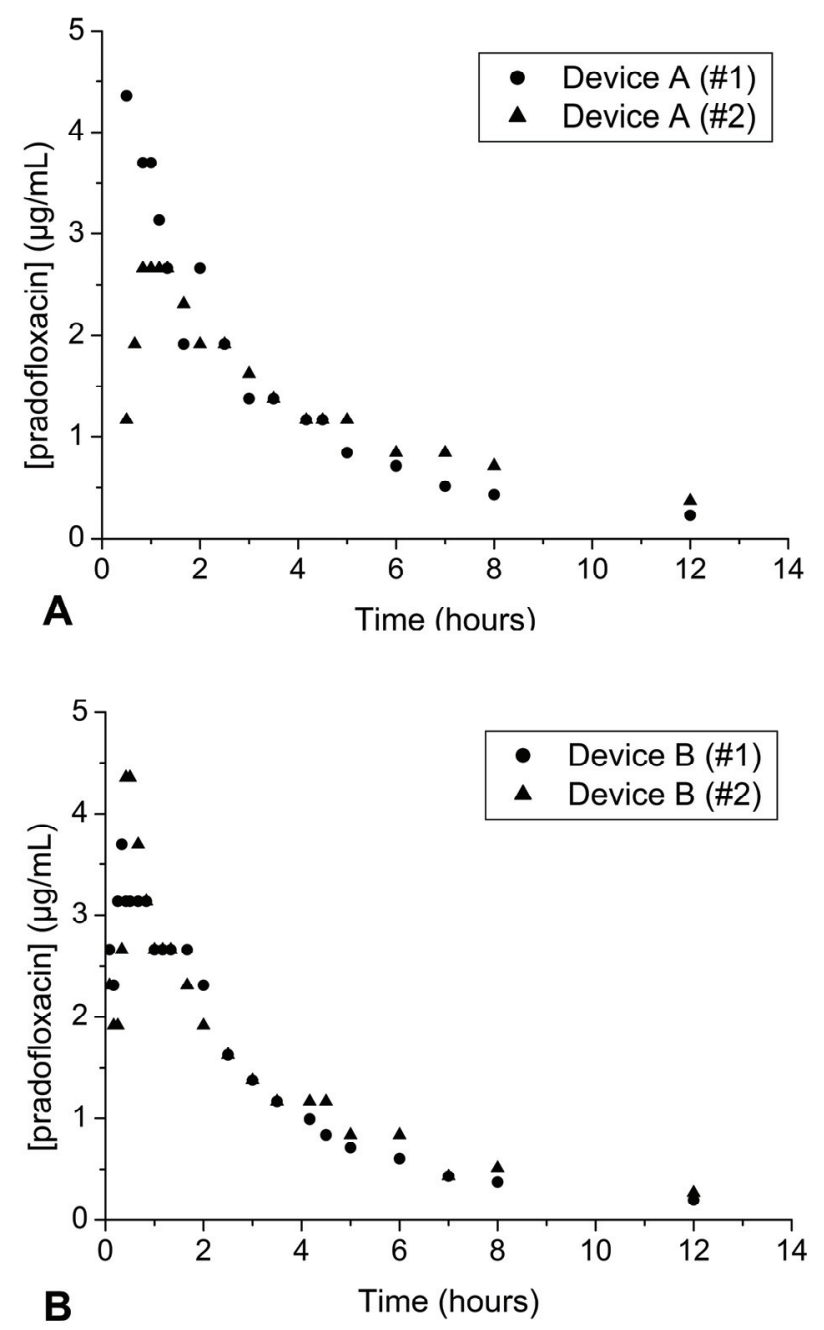

Figure 6.5A. In vitro release from device A (i.e. pradofloxacin in the coil's lumen, but not in the surface coating). B. Device B (i.e. pradofloxacin in the lumen and in the surface coating). 
In the beginning, the release from device A shows some variations probably due to wetting of the coil. As expected, device B released more pradofloxacin during the first hour at least.

The amount of pradofloxacin released was estimated by calculating the area under the curve (AUC) of the release plot ([pradofloxacin] versus elution volume). For device A, the AUC was 89 and $91 \mu \mathrm{g}$, and for device B, we measured 130 and $176 \mu \mathrm{g}$. Integration of the release curves for device B was less accurate due to substantial spreading in the first data points. The fraction of the entrapped drug released from the coil coating was approximately $45 \%$, and the fraction released from the rod is approximately $80 \%$.

The data revealed that approximately $60 \mu \mathrm{g}$ of pradofloxacin was released from the surface coating. In other words, this implies that the OphthaCoil's drug capacity was increased by a factor 2.5 by using the pradofloxacin-loaded polyHEMA rod in the lumen of the device.

For the bacterium E. coli ATCC 25922, the minimum inhibitory concentration (MIC) of pradofloxacin, defined as the lowest drug concentration inhibiting visible growth, is 0.008 $0.015 \mu \mathrm{g} / \mathrm{mL}$ and the mutant prevention concentration (MPC), defined as the drug concentration allowing no visible residual growth, is $0.075-0.1 \mu \mathrm{g} / \mathrm{mL}$ [15]. After $32 \mathrm{~h}$, the concentration measured in the collected fraction was $0.08 \mu \mathrm{g} / \mathrm{mL}$ (not shown in Figure 6.5 to clarify the differences in the first hours of the release). This is equal to the mutant prevention concentration of pradofloxacin for E. coli ATCC 25922 and approximately ten times higher than the minimum inhibitory concentration. This means that the amount of pradofloxacin released after $32 \mathrm{~h}$ is still sufficient to inhibit growth of the E. coli strain used.

\subsubsection{In vivo release}

Pradofloxacin was detectable from the beginning of the sampling period until the end at $72 \mathrm{~h}$ post-application (Figure 6.6). Although there were some individual variations, a clear and consistent release pattern was found. The curves are reminiscent of the release curves that were obtained in vitro, albeit that the in vivo release was substantially lower. Drug levels in the tear fluid declined steadily, but they always remained within the concentration range where pradofloxacin is expected to be active against the most relevant bacteria species involved in canine conjunctival infections.

There was no sign of bacteria inhibition from the control measurements, anesthetics, or blank disc, except for the anesthetic Antisedan, which, after being diluted ten times, showed no more bacteria inhibition. Because Antisedan was administered systemically, it can be assumed that the drug is widely distributed and diluted in the body. Therefore, it can be excluded that Antisedan was able to reach the lacrimal glands in concentrations high enough to produce false-positive reactions in the bioassay. 


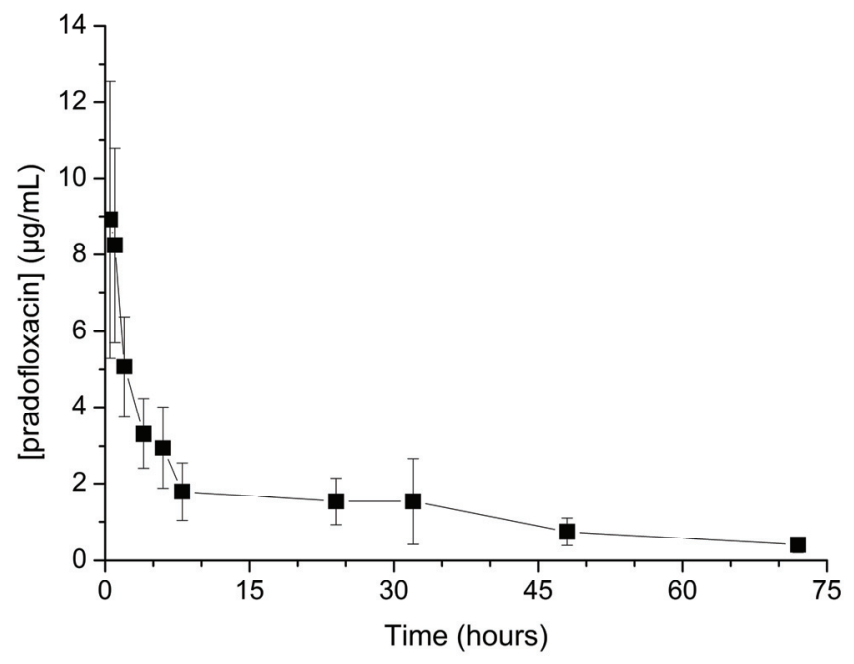

Figure 6.6. Arithmetic group means of tear fluid concentrations of pradofloxacin in both eyes of Beagle dogs.

The in vivo measurements show a longer release time compared to the in vitro data, i.e. $72 \mathrm{~h}$ compared to $32 \mathrm{~h}$. This may be attributed to the relative slow refresh rate of tear fluid in the eye. The in vitro experiments were performed with a flow of $100 \mu \mathrm{L} / \mathrm{min}$, whereas the production rate of tear fluid is only $1-1.2 \mu \mathrm{L} / \mathrm{min}$ for non-stimulated tears and $5 \mu \mathrm{L} / \mathrm{min}$ for stimulated tears [20]. We chose to set the flow rate at $100 \mu \mathrm{L} / \mathrm{min}$ because samples are collected very slowly at slower flows, and measurements are precluded by fluid evaporation.

Based on this data, we would like to make the following remarks. First, we realize that it is intuitively strange to use a metallic component in an ocular insert. However, the coiled wire in the OphthaCoil has several essential functions. The metal is never in contact with any ocular tissue. The wire serves as a stable base for the hydrogel coating, and it ensures the structural integrity of the device during application. This implies that the OphthaCoil can be removed from the conjunctival fornix in one piece. In addition, the OphthaCoil can be removed from the fornix using a small magnet instead of tweezers, and the device can be seen in situ through Xray fluoroscopy. This is shown in Figure 6.7. The X-ray visibility of the device can be an important safety feature. 


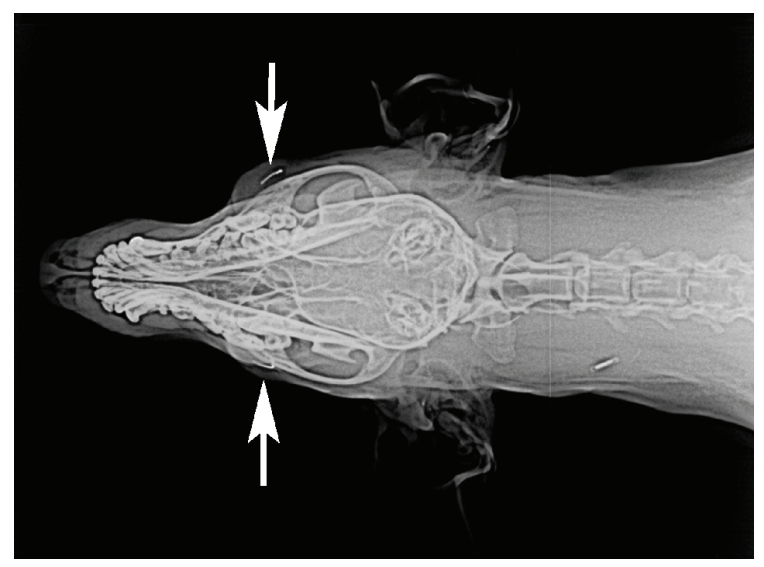

Figure 6.7. X-ray image of the head of a Beagle dog in ventro-dorsal projection. The dog has an

OphthaCoil in the lower conjunctival fornix of both eyes (see arrows).

Second, in device B the drug pradofloxacin was released from two different depots: the surface coating and the polyHEMA hydrogel rod in the lumen. This opens up the possibility of delivering two different drugs from one device: one drug can be released almost immediately after installation from the surface coating, and another drug can be released more slowly and steadily from the inside of the OphthaCoil. This feature provides many different options for designing a drug delivery device in terms of drugs, doses, and release kinetics.

Third, in our in vivo experiments it was necessary to fasten the OphthaCoil in the conjunctival fornix with a single stitch. This is obviously not an ideal situation. In previous experiences, the OphthaCoil did not stay in the Beagles' eyes overnight, probably because of their third eyelid that covers most of the cornea during sleep [10]. We found that attaching the OphthaCoil prevents this problem. We assume that the OphthaCoil will remain in the conjunctival fornix in human patients who do not have a third eyelid; however, it must be noted that this has not yet been verified. In veterinary medicine, conjunctivitis, keratoconjunctivitis sicca (KCS) and corneal ulcers are the most common eye disorders found in animals [21, 22]. In treating animal diseases, it may (at least in certain cases) be acceptable to fasten the ocular device with a stitch. If a biodegradable suture such as Vicryl is used, the contact time of the OphthaCoil may be controlled. It should be noted that the use of a sustained delivery device to administer cyclosporine to horse eyes has been described [23]. A tubular reservoir loaded with the drug was implanted in the suprachoroidal space, which required general anesthesia. Then, cyclosporine was released for approximately 24 months. However, the reservoir is a permanent implant that is not removed after it is empty. We speculate that the OphthaCoil could 
eventually become an alternative to this approach with the potential advantages that installation of the OphthaCoil is much easier, and that the device can be removed from the eye after a certain period of time due to the degradation of the suture material.

\subsection{Conclusions and perspectives}

Based on the current data, we believe that the OphthaCoil holds promise as a new device for controlled delivery of one or two drugs to the eye. The device has several pros and cons that need to be carefully weighed for several potential applications. The OphthaCoil may be useful to treat (severe) ocular infections in human patients or to administer different drugs (e.g. a mydriatic agent and an antibiotic) prior to cataract operation. In veterinary medicine, the OphthaCoil may be used to deliver a drug to the eye for an extended period of time in cases such as KCS or corneal ulcers. In these types of applications, it will probably be necessary to attach the device to the conjunctival fornix. Further work to tailor the OphthaCoil for specific applications in human and veterinary ophthalmology is currently in progress in our laboratories.

\subsection{Acknowledgements}

This work was carried out with the financial support of DSM Research (Geleen, the Netherlands).

\section{References}

1. Hosoya K., Lee V.H., and Kim K.J. Roles of the conjunctiva in ocular drug delivery: a review of conjunctival transport mechanisms and their regulation. Eur J Pharm Biopharm 60 (2) (2005) 227240.

2. Gulsen D., and Chauhan A. Ophthalmic drug delivery through contact lenses. Invest Ophthalmol Vis Sci 45 (7) (2004) 2342-2347.

3. Le Bourlais C., Acar L., Zia H., Sado P.A., Needham T., and Leverge R. Ophthalmic drug delivery systems - recent advances. Prog Retin Eye Res 17 (1) (1998) 33-58.

4. $\quad$ Barbu E., Sarvaiya I., Green K.L., Nevell T.G., and Tsibouklis J. Vinylpyrrolidone-co-(meth)acrylic acid inserts for ocular drug delivery: Synthesis and evaluation. J Biomed Mater Res A 74 (4) (2005) 598-606.

5. Kaur I.P., and Kanwar M. Ocular preparations: the formulation approach. Drug Dev Ind Pharm 28 (5) (2002) 473-493. 
6. Mah F.S. Fourth-generation fluoroquinolones: new topical agents in the war on ocular bacterial infections. Curr Opin Ophthalmol 15 (4) (2004) 316-320.

7. Mather R., Karenchak L.M., Romanowski E.G., and Kowalski R.P. Fourth generation fluoroquinolones: new weapons in the arsenal of ophthalmic antibiotics. Am J Ophthalmol 133 (4) (2002) 463-466.

8. Linde H.J., and Lehn N. Mutant prevention concentration of nalidixic acid, ciprofloxacin, clinafloxacin, levofloxacin, norfloxacin, ofloxacin, sparfloxacin or trovafloxacin for Escherichia coli under different growth conditions. J Antimicrob Chemother 53 (2) (2004) 252-257.

9. Pijls R.T., Hanssen H.H., Nuijts R.M., and Koole L.H. Flexible coils with a drug-releasing hydrophilic coating: A new platform for controlled delivery of drugs to the eye? Bio-Med Mater Eng 14 (4) (2004) 383-393.

10. Pijls R.T., Sonderkamp T., Daube G.W., Krebber R., Hanssen H.H., Nuijts R.M., and Koole L.H. Studies on a new device for drug delivery to the eye. Eur J Pharm Biopharm 59 (2) (2005) 283-288.

11. Aldenhoff Y.B., Knetsch M.L., Hanssen J.H., Lindhout T., Wielders S.J., and Koole L.H. Coils and tubes releasing heparin. Studies on a new vascular graft prototype. Biomaterials 25 (16) (2004) 31253133.

12. Hanssen H.H., Wetzels G.M., Benzina A., van der Veen F.H., Lindhout T., and Koole L.H. Metallic wires with an adherent lubricious and blood-compatible polymeric coating and their use in the manufacture of novel slippery-when-wet guide wires: possible applications related to controlled local drug delivery. J Biomed Mater Res 48 (6) (1999) 820-828.

13. Knetsch M.L., Aldenhoff Y.B., Schraven M., and Koole L.H. Human endothelial cell attachment and proliferation on a novel vascular graft prototype. J Biomed Mater Res 71A (4) (2004) 615-624.

14. Peerlings C.C., Hanssen H.H., Bevers R.T., Boelen E.J., Stelt B.J., Korthagen E.J., and Koole L.H. Heparin release from slippery-when-wet guide wires for intravascular use. J Biomed Mater Res 63 (6) (2002) 692-698.

15. Wetzstein H.G. Comparative mutant prevention concentrations of pradofloxacin and other veterinary fluoroquinolones indicate differing potentials in preventing selection of resistance. Antimicrob Agents Chemother 49 (10) (2005) 4166-4173.

16. Gulsen D., and Chauhan A. Dispersion of microemulsion drops in HEMA hydrogel: a potential ophthalmic drug delivery vehicle. Int J Pharm 292 (1-2) (2005) 95-117.

17. Ferreira L., Vidal M.M., and Gil M.H. Evaluation of poly(2-hydroxyethyl methacrylate) gels as drug delivery systems at different pH values. Int J Pharm 194 (2) (2000) 169-180.

18. Kejlova K., Labsky J., Jirova D., and Bendova H. Hydrophilic polymers - biocompatibility testing in vitro. Toxicol In Vitro 19 (7) (2005) 957-962.

19. Paulsson M., and Edsman K. Controlled drug release from gels using surfactant aggregates I: Effect of lipophilic interactions for a series of uncharged substances. J Pharm Sci 90 (9) (2001) 1216 1225 .

20. Glasson M.J., Stapleton F., Keay L., Sweeney D., and Willcox M.D. Differences in clinical parameters and tear film of tolerant and intolerant contact lens wearers. Invest Ophthalmol Vis Sci 44 (12) (2003) 5116-5124.

21. Baeyens V., Felt-Baeyens O., Rougier S., Pheulpin S., Boisrame B., and Gurny R. Clinical evaluation of bioadhesive ophthalmic drug inserts (BODI) for the treatment of external ocular infections in dogs. J Control Release 85 (1-3) (2002) 163-168.

22. Baeyens V.V., Percicot C., Zignani M., Deshpande A.A., Kaltsatos V.V., and Gurny R. Ocular drug delivery in veterinary medicine. Adv Drug Deliv Rev 28 (3) (1997) 335-361.

23. Gilger B.C. Equine Ophthalmology, ed., Elsevier Saunders (2005) 313-314. 


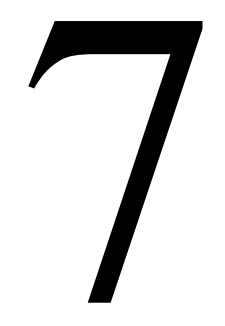

The OphthaCoil for treatment of infections or pre-operative delivery of drugs to the eye

Rachel T. Pijls, Peter P.K.H. Fransen, Hans H.L. Hanssen, Sandra Lindemann, Christiane Smetonat, Gert W. Daube, and Leo H. Koole, to be submitted 


\section{Abstract}

The in vitro release of the drugs chloramphenicol and cyclosporine A from the OphthaCoil, an ocular drug delivery device, was evaluated. The ocular device consists of a hydrogel coated coil, where the drug can be entrapped. The device can be placed in the lower conjunctival fornix, where the drug is released. The capacity of the device could be increased by two different methods of filling the interior of the coil: a polymer rod, polymerized in the presence of the drug, or a textile fiber, soaked into a highly concentrated solution of the drug. The in vitro release of chloramphenicol shows that using the polymer rod, the capacity could be increased by almost 300\%, however using the fiber, the capacity was increased for appr. 500\%, all compared to a coil with an empty interior.

Also, an evaluation of the mydriatic effect in the eyes of Beagle dogs was done. A device, filled with a textile fiber soaked into a solution of phenylephrine hydrochloride and tropicamide, was placed in the left eye of eight dogs. The response of the pupil to light was observed, and within 30 minutes to 1 hour, a delayed or no response at all was seen in all canine eyes, which persisted for $4 \mathrm{~h}$ at least. Pupil dilation was clearly visible in all animals within 30 minutes to 1 hour. Again, the effect lasted for $4 \mathrm{~h}$ at least.

Finally, larger coils were prepared for the insertion in the equine eye. In vitro release of cyclosporine A showed that the capacity of the device could be increased drastically with the use of the polymer rod or the textile fiber (with a factor 10 and 18 respectively, both compared to an empty coil). The release decreases gradually over time for at least 72 hours. Insertion of the device in the equine eye was uneventful and easy, without the use of local or systemic anaesthetics $(n=2)$. The horses tolerated the devices very well. After $5 \mathrm{~h}$, all coils were still in place, however after $24 \mathrm{~h}$, none of them could be recovered. 


\subsection{Introduction}

The conventional method of topical drug delivery to the eye has well-known drawbacks. The absorption of the drug to the site of action is very limited, partly due to solubility of the drug, elimination and spilling of the eye drop [1,2]. As a result, frequent instillations are necessary, which may cause toxic side-effects and poor patient compliance [3, 4]. Ocular inserts can prevent most of those problems [5]. An insert can release its drug gradually and more controlled, resulting in a significant increase of the bio-availability, defined as the rate and extent of absorption of the drug [6]. An insert provides a reduction of systemic absorption as well, and the number of applications can be minimized $[2,7]$.

Most ocular inserts that have been described so far are based on hydrogel technology. The drug is blended with a hydrogel, and the construct is applied in dry form. After application in, for example, the lower conjunctival fornix, the insert will swell through absorption of tear fluid, and the drug is released concomitantly $[8,9]$. Next, advances of an ocular insert in three different applications are addressed.

First, in the treatment of (severe) bacterial infections of the conjunctiva or cornea, a broad spectrum antibiotic or antiinfective is used, for example the drugs chloramphenicol and gentamicin $[10,11]$. In bacterial conjunctivitis, 1-2 eye drops of chloramphenicol have to be administered topically to the eye up to eight times a day [12]. In severe infections, the frequency can be increased up to 1-2 drops every 15-20 minutes, until the infection is controlled, after which the frequency can be gradually reduced. The prevalence of (severe) bacterial infections is increasing with the use of long-term wear contact lenses [13-15]. An ocular insert can ensure more controlled drug delivery to the eye, as well as minimizing the frequency of instillations drastically.

Second, over the past decade, evidence suggests that dry eye syndrome (kerato-conjunctivitis sicca) is the result of an underlying inflammatory process [16, 17]. In 2002, cyclosporine A has been approved by the US Food and Drug Administration for the treatment of the inflammatory process in dry eye disease $[18,19]$. An ocular device could be placed in the conjunctival fornix, possibly without the use of local anaesthetics, where it can release its drug to the tear film for a period of time.

Third, an ocular device can be used for the pre-operative delivery of drugs to the eye. For example, before cataract surgery, which accounts for over $75 \%$ of all eye operations in hospitals in the Netherlands [20]. Before cataract surgery, where the natural lens is replaced by an intraocular lens, three types of drugs must be administered: an antibiotic, two mydriatic 
agents, and an anaesthetic agent. An ocular device containing a combination of these drugs would be an advantage over multiple instillations of eye drops.

In our previous work, we introduced a new ocular drug delivery device, the OphthaCoil. Hydrogel technology also provides the basis for this device. The hydrogel biomaterial is present as a thin adherent coating on the exterior of the OphthaCoil. The coating is blended with an active substance, such as the antibiotics chloramphenicol and pradofloxacin [21-23]. Based on all available data, we firmly believe that the OphthaCoil will turn out to be useful in the three indications outlined above. Here, we wish to report progress on the development of the insert in two directions: (i) increase of the drug capacity (and time-window of action) by new methods to utilise the inner space of the coiled structure; (ii) development of an enlarged version of the OphthaCoil, and preliminary studies of the potential utility of the large OphthaCoil to deliver drug to the equine eye. We report further technical details on several prototype OphthaCoils with enhance drug loading (in the interior and/or in the exterior coating), as well as results from in vivo experiments with dogs (Beagles) and horses.

\subsection{Materials and methods}

\subsubsection{Preparation of the devices}

All chemicals were purchased from Sigma-Aldrich (Steinheim, Germany), unless stated otherwise. The ocular devices were prepared as described earlier [24, 25]. Briefly, the hydrogel Slipskin $^{\circledR}$ (SS) was prepared by free radical bulk polymerization of the hydrophilic monomer $\mathrm{N}$-vinyl pyrrolidone (NVP), and the hydrophobic monomer butyl methacrylate (BMA), in a molar ratio of 70/30. The initiator $\alpha, \alpha^{\prime}$-azoisobutyronitrile (AIBN) was used here, and the polymerization resulted in hard, opaque rods. The polymer was dissolved in the solvent Nmethyl pyrrolidone (NMP). This mixture was used for the coating procedure. Here, two stainless steel wires, with diameters of 76 and $176 \mu \mathrm{m}$, were used. The wires were coated twice with the mixture, and the coated wires were coiled around core wires using a speed controllable electromotor. The coated wire of $76 \mu \mathrm{m}$ was coiled around a wire with a diameter of $432 \mu \mathrm{m}$, and the other coated wire was coiled around a wire of $870 \mu \mathrm{m}$ in diameter. Coils of approximately $50 \mathrm{~cm}$ in length were prepared.

In addition, coated wires with a drug-containing coating were prepared. In the solvent NMP, SS and the antibiotic chloramphenicol (CAP) (50/50 w/w\%) or SS and the immunosuppressive agent cyclosporine A (CsA, LC Laboratories, Woburn, USA) (70/30 w/w\%) were dissolved. 
The mixture containing CAP was coated onto a wire of $76 \mu \mathrm{m}$ and coiled around a core wire of $432 \mu \mathrm{m}$. And the other mixture with CsA was coated onto a $176 \mu \mathrm{m}$ wire and coiled around a core wire of $870 \mu \mathrm{m}$. The inner core wire was later removed, so that a flexible coil was obtained. The coils were cut into lengths of $15 \mathrm{~mm}$ for the smaller diameter coils, and $30 \mathrm{~mm}$ for the larger coils.

Table 7.1. The different coils prepared.

\begin{tabular}{|c|c|c|c|c|}
\hline Coil & $\begin{array}{l}\text { Diameter } \\
\text { wire }(\mu \mathrm{m})\end{array}$ & $\begin{array}{l}\text { Inner diameter } \\
\text { coil }(\mu \mathrm{m})\end{array}$ & $\begin{array}{l}\mathrm{w} \% \text { drug in } \\
\text { coating }\end{array}$ & Interior of the coil \\
\hline $\mathrm{A}_{0}$ & 76 & 432 & $50 \mathrm{w} \% \mathrm{CAP}$ & - \\
\hline $\mathrm{A}_{1}$ & 76 & 432 & - & PolyHEMA-rod + $5.0 \mathrm{w} \%$ CAP \\
\hline $\mathrm{A}_{2}$ & 76 & 432 & $50 \mathrm{w} \% \mathrm{CAP}$ & PolyHEMA-rod $+5.0 \mathrm{w} \%$ CAP \\
\hline $\mathrm{A}_{3}$ & 76 & 432 & - & Textile fiber soaked into \\
\hline $\mathrm{A}_{4}$ & 76 & 432 & $50 \mathrm{w} \% \mathrm{CAP}$ & $\begin{array}{l}100 \mathrm{mg} / \mathrm{mL} \text { CAP in ethanol } \\
\text { Textile fiber soaked into } \\
100 \mathrm{mg} / \mathrm{mL} \mathrm{CAP} \mathrm{in} \mathrm{ethanol}\end{array}$ \\
\hline $\mathrm{B}_{0}$ & 176 & 870 & $30 \mathrm{w} \% \mathrm{CsA}$ & - \\
\hline $\mathrm{B}_{1}$ & 176 & 870 & - & PolyHEMA-rod + $10.0 \mathrm{w} \%$ CsA \\
\hline $\mathrm{B}_{2}$ & 176 & 870 & $30 \mathrm{w} \% \mathrm{CsA}$ & PolyHEMA-rod + $10.0 \mathrm{w} \%$ CsA \\
\hline $\mathrm{B}_{3}$ & 176 & 870 & - & $\begin{array}{l}\text { Textile fiber soaked into } \\
160 \mathrm{mg} / \mathrm{mL} \text { CsA in ethanol }\end{array}$ \\
\hline $\mathrm{B}_{4}$ & 176 & 870 & $30 \mathrm{w} \% \mathrm{CsA}$ & $\begin{array}{c}\text { Textile fiber soaked into } \\
160 \mathrm{mg} / \mathrm{mL} \text { CsA in ethanol }\end{array}$ \\
\hline $\mathrm{C}$ & 76 & 432 & - & $\begin{array}{c}\text { Textile fiber soaked into } 100 \mathrm{mg} / \mathrm{mL} \\
\text { PHE and TR }(5 / 1 \mathrm{w} / \mathrm{w}) \text { in ethanol }\end{array}$ \\
\hline
\end{tabular}

The interior of the coils were filled to increase the capacity of the device and to extend the release time of the drugs, as described in our previous work [21, 22]. Two different techniques were used. First, the hydrogel poly(2-hydroxyethyl methacrylate) (polyHEMA) was prepared in the presence of the drug CAP or CsA ( $5.0 \mathrm{w} \%$ and $10.0 \mathrm{w} \%$ respectively). As initiator, $3.0 \%$ AIBN was used. The hydrogels were polymerized at $80^{\circ} \mathrm{C}$ in silicon tubes with an inner diameter of $0.4 \mathrm{~mm}$ and $0.6 \mathrm{~mm}$ for the drugs CAP and CsA respectively. The hydrogels were removed from the tube, cut into pieces of $13 \mathrm{~mm}$ or $28 \mathrm{~mm}$ depending on the size of the coil, 
and placed inside the coils. The ends of the coils were closed using a UV curable cyanoacrylate.

In second approach, textile fibers were soaked into a solution of $100 \mathrm{mg} / \mathrm{mL}$ CAP in ethanol or $160 \mathrm{mg} / \mathrm{mL}$ CsA in ethanol. The fibrils were dried in open air for 24 hours and were pulled inside the coils. Again, the ends of the coils were closed. Additionally, textile fibers were soaked into a solution containing $100 \mathrm{mg} / \mathrm{mL}$ phenylephrine hydrochloride (PHE, Acros Organics, Geel, Belgium) and tropicamide (TR) in a ratio of 5:1 in ethanol. After drying, the fibrils were placed into a coil coated with only the hydrogel SS and the ends were closed again.

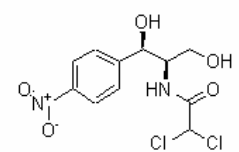

Chloramphenicol

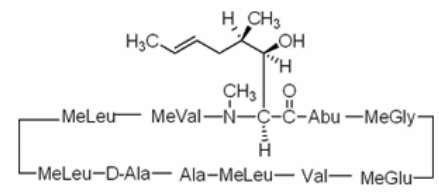

Cyclosporine A

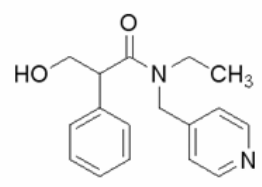

Tropicamide

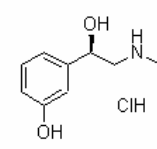

Phenylephrine hydrochloride

Figure 7.1. The chemical structures of the active agents used.

The chemical structures of the active agents are given in Figure 7.1. All prepared devices are listed in Table 7.1. In summary, the series A have an inner diameter of $432 \mu \mathrm{m}$ and contain the drug CAP; the series B have an inner diameter of $870 \mu \mathrm{m}$ and contain the drug CsA. The numbers 0 have the drug in the coating of the coil, but none in the interior. The numbers 1 and 3 have a non-loaded coated coil with, respectively, a drug-loaded polymer rod or drug-loaded textile fibers in the interior. And the numbers 2 and 4 have both a drug-loaded coated coil as well as a drug-loaded polymer (nr. 2) or drug-loaded textile fibers (nr. 4) in the interior of the coil. Finally, coil $\mathrm{C}$ contains the textile fibers soaked into the mydriatic agents.

\subsubsection{Drug measurements}

High performance liquid chromatography (HPLC) was used to measure the concentration of the drugs in the samples. For the drug chloramphenicol, a Hewlett Packard HP 1090 HPLC apparatus equipped with a C18 column 150 x $4.6 \mathrm{~mm}$ (X Terra RP 18, Waters, Eschborn, Germany) was used with a mean particle size of $3.5 \mu \mathrm{m}$. As eluents, a $10 \mathrm{mM}$ phosphoric acid / water solution (phase A) and acetonitrile (phase B) were used. The temperature was set at $40^{\circ} \mathrm{C}$, and the software used was ChemStation software version Rev. A.08.03 (Agilent Technologies, Amstelveen, the Netherlands). 
In the gradient cycle of 15 minutes, the mobile phase was changed from $10 \%$ to $70 \%$ of the mobile phase B over a period of 10 minutes; kept at $70 \%$ for 1 minute, and thereafter changed to $90 \%$ of the mobile phase B over a period of 0.1 minute; again kept at $90 \%$ for 1 minute, and lowered to $10 \%$ in 0.1 minute, where it was kept until the next sample was injected. A flow rate of $1.5 \mathrm{~mL} / \mathrm{min}$, and an injection volume of $150 \mu \mathrm{L}$ were used during the analysis. The detection was done at a wavelength of $280 \mathrm{~nm}$. The drug concentration in the samples could be calculated using a dilution series with samples of $0.10-45 \mu \mathrm{g} / \mathrm{mL}$ chloramphenicol in water.

For the detection of the drug CsA, a Varian ProStar 215 SDM with a C4 column $150 \times 4.6 \mathrm{~mm}$ (Grace Vydac, Deerfield, USA) was used. As eluents, buffer A contained $99.9 \% \mathrm{H}_{2} \mathrm{O}$ and $0.1 \%$ trifluoric acid (TFA), and buffer B contained $89.95 \%$ acetonitrile (ACN), 9.95\% $\mathrm{H}_{2} \mathrm{O}$ and $0.1 \%$ TFA. The measurements were done at room temperature.

In the gradient cycle, the ratio of buffer B was steadily increased from $60 \%$ to $90 \%$ over 15 minutes, where it was kept for 5 minutes. Thereafter, the ratio was decreased to $60 \%$ in one minute, and finally decreased slowly to $55 \%$ in the final 9 minutes of the cycle. A flow rate of $1.0 \mathrm{~mL} / \mathrm{min}$ and an injection volume of $200 \mu \mathrm{L}$ were used. The detection was done at a wavelength of $210 \mathrm{~nm}$. The drug concentration in the samples could be calculated using a dilution series with samples of $0.5-40 \mu \mathrm{g} / \mathrm{mL}$ CsA in 1:1 (v/v) ethanol/simulated tear fluid (STF [26]).

\subsubsection{Drug release in vitro}

\subsubsection{Chloramphenicol release}

The release of different coils, containing the drug chloramphenicol was measured in demineralized water at room temperature. The coils were placed in a vial with $10 \mathrm{~mL}$ of water. After 24 hours, $150 \mu \mathrm{L}$ of the sample solution was used for the HPLC detection method. Coils $A_{0}-A_{4}$ were used for the release experiment $(n \geq 10)$.

\subsubsection{Cyclosporine A release}

The release of the drug cyclosporine A from different coils was measured in STF. The coils were placed in a vial with $50 \mathrm{~mL}$ of STF. Coils $\mathrm{B}_{0}-\mathrm{B}_{4}$ were used for the release experiment (n $=3$ ). After 4 and 7 days of immersion, the sample medium was refreshed. And the collected samples were diluted 1:1 with ethanol for consistency with the calibration curve. The samples 
were stored at $4^{\circ} \mathrm{C}$ until measurement using the HPLC method described above. The cumulative amount of CsA released was calculated.

A second experiment with the coils was performed in a flow setup. STF was perfused through a silicon tube (inner diameter of $1.6 \mathrm{~mm}$ ) with a rate of $0.01 \mathrm{~mL} / \mathrm{min}$. Coil $\mathrm{B}_{2}$ and $\mathrm{B}_{4}$ were used. They were placed at the end to the tube, and samples of $0.1 \mathrm{~mL}$ were collected at different time points and diluted with $0.1 \mathrm{~mL}$ ethanol $(\mathrm{n}=3)$. Again, the samples were stored at $4^{\circ} \mathrm{C}$ until measurement.

\subsubsection{In vivo experiments}

In vivo studies were carried out at Bayer HealthCare AG (Animal Center, Monheim, Germany) on their dogs and horses. Prior to the in vivo experiments, a clinical examination was performed. Each animal was examined for general condition (posture/behaviour), gingivae, haircoat and skin, respiratory tract, cardiovascular tract, digestive tract, urinary and reproductive system, musculoskeletal system, CNS and ocular system. The exclusion criteria were growing animals, pregnant or nursing animals, and animals with a disturbed state of health and/or symptoms of a considerable disease, especially of the ocular system. Furthermore, a final clinical examination was done 24 hours after application of the devices. The examinations followed standard veterinary procedures by the veterinarian in charge.

\subsubsection{Mydriasis in dogs}

An OphthaCoil was inserted into one eye of eight Beagle dogs to evaluate the mydriatic effect of phenylephrine and tropicamide to the canine eye. Coil $\mathrm{C}$ was used for the experiment, i.e. the hydrogel coating did not contain a drug, whereas the interior was filled with textile fibers soaked into the mydriatic agents. For the study, eight adult Beagle dogs ( 7 female, 1 male) were used. They were $1-2$ years old and the body weight ranged between 8 and $12 \mathrm{~kg}$. The animals were housed individually in pens. The floor consisted of tiles. Relative humidity ranged between $50-60 \%$ and the temperature varied between $15-21^{\circ} \mathrm{C}$. Lighting was controlled with approximately 12 hours of artificial light from $6.00 \mathrm{am}$ to $6.00 \mathrm{pm}$. Once a day, the dogs were fed a commercially available dry dog food. Water was provided for ad libitum consumption.

All eight animals received a coil in the lower conjunctival fornix of the left eye. The coils were inserted with a pair of tweezers. The eyes of the dogs were examined to evaluate the mydriatic effects of the coils at the following time points: prior to insertion, 1/2, 1, 2, 3, 4, 5, 6, 7 and 24 hours after insertion of the device. The following parameters were monitored: 
- presence of the coil in the left eye

- response of the pupil to light $(1=$ normal reaction, $2=$ delayed reaction, $3=$ no reaction $)$

- dilation of the pupil ( 1 = no dilation, 2 = moderate dilation, 3 = full dilation)

The coils were removed after 6 hours, if they were not lost spontaneously.

\subsubsection{Tolerance evaluation in horses}

To evaluate the persistence and the ease of application of the OphthaCoil in the eye of horses, an OphthaCoil of $30 \mathrm{~mm}$ in length was placed in the lower conjunctival fornix of the equine eye. Coil $\mathrm{B}_{4}$ was used for the experiment.

For the study, two adult horses (1 male, 1 female) were selected. The animals were housed individually in stables with straw bedding. Over the day, they have been kept in outdoor paddocks with a floor of laminated grids. Once a day, the horses were fed hay and a commercially available pellet horse food. Water supply was provided for ad libitum consumption by automatic water dispensers.

Two animals received a coil in the lower conjunctival fornix of the left eye. The coils were inserted with a pair of tweezers. The horses were observed directly after the application for their immediate reaction to the coil. The eyes were examined to evaluate the presence of the coil at the following time points: 3 hours, 5 hours, and 24 hours after application. It was planned to remove the coils after 24 hours of application, if still present.

\subsection{Results and Discussion}

\subsubsection{Preparation of the coils}

All devices listed in Table 7.1 could be prepared successfully. Coils from series A and C weighed $10-15 \mathrm{mg}$, and coils from series B weighed approximately $130 \mathrm{mg}$. Figure 7.2 shows a photograph of coil $\mathrm{A}_{0}$ and $\mathrm{B}_{0}$. 


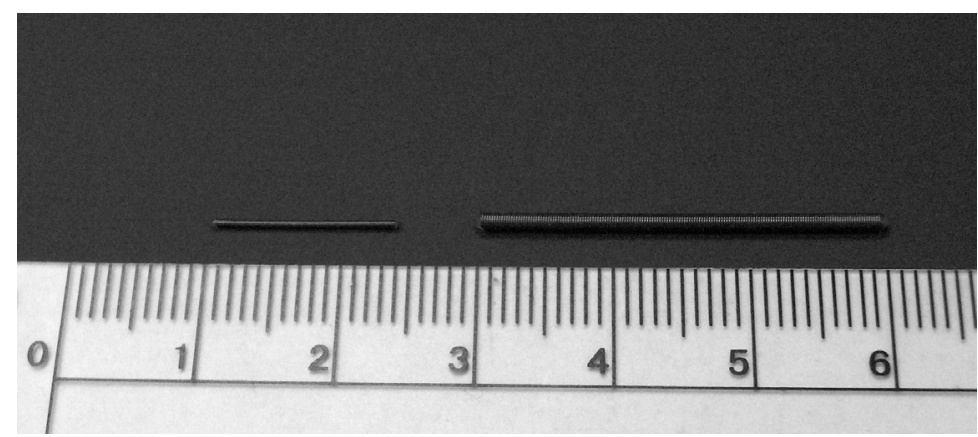

Figure 7.2. Photographs of two different coils (coil $A_{0}$ and $B_{0}$ ).

\subsubsection{Drug release in vitro}

\subsubsection{Chloramphenicol release}

Figure 7.3 shows the total release of chloramphenicol from the coils $\mathrm{A}_{0}-\mathrm{A}_{4}$. Coil $\mathrm{A}_{0}$ released $53 \mu \mathrm{g}$, which is $49 \%$ of the drug entrapped in the hydrogel coating. Filling the interior of the coil, does improve the total release of the drug. Coils $\mathrm{A}_{1}$ and $\mathrm{A}_{2}$, which contained the hydrogel rod, released $161( \pm 15.9)$ and $198( \pm 21.2) \mu \mathrm{g}$ respectively. And coils $\mathrm{A}_{3}$ and $\mathrm{A}_{4}$, which contained the textile fibers, released $216( \pm 68.5)$ and $312( \pm 79.6) \mu \mathrm{g}$ respectively. It shows that using the polymer rod, the capacity could be increased by almost 300\%, however using the fibers, the capacity was increased for appr. 500\%, both compared to a coil with an empty interior.

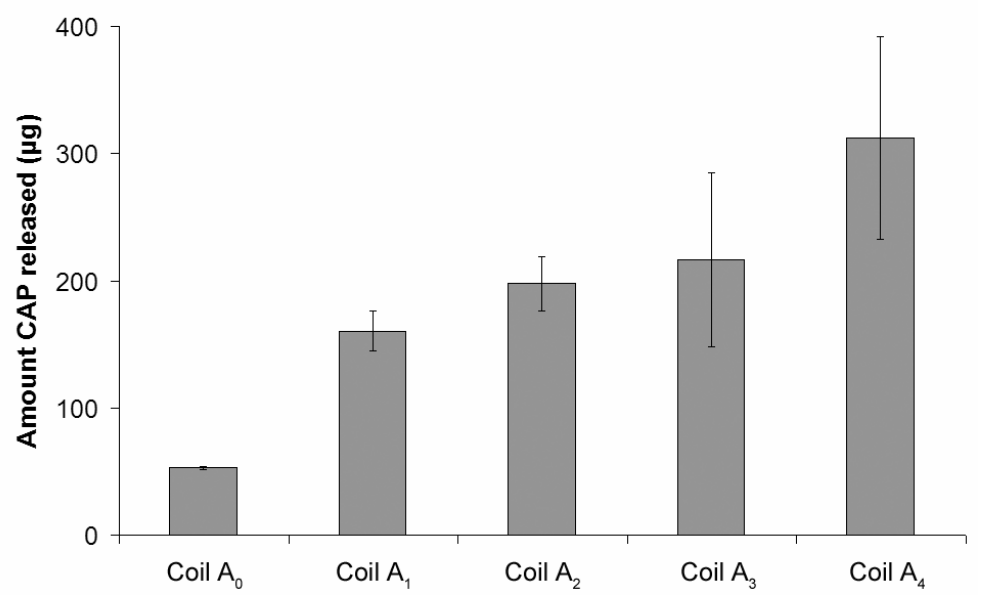

Figure 7.3. The amount of chloramphenicol released from different types of devices $(n \geq 10)$. 
We noticed, however that it is difficult to achieve truly reproducible drug release profiles with coils filled with the textile fibers. This may indicate that further improvements in our methods to impregnate the drug in the fiber and/or to fill the device, are required.

\subsubsection{Cyclosporine A release}

First release experiments in vials, showed that $53 \mu \mathrm{g}$ (which is $22 \%$ ) of the cyclosporine A entrapped in the coating was released. At first, the results from coils $\mathrm{B}_{0}, \mathrm{~B}_{2}$, and $\mathrm{B}_{4}$ were similar, what indicates that no drug was released from the interior of the coil. An explanation could be the fact that the drug is very hydrophobic and the molecule size is too large to diffuse through the windings of the coil. Therefore, the windings of the coils were expanded, such that the release medium could easily diffuse into the coil and the drug could diffuse out of the coil. Figure 7.4 shows the total cumulative release of the coils $\mathrm{B}_{0}-\mathrm{B}_{4}$ in STF after expansion of the windings of all coils. Coils $\mathrm{B}_{1}$ and $\mathrm{B}_{2}$, which contained the hydrogel rod, released $285( \pm 70.5)$ and $532( \pm 70.5) \mu \mathrm{g}$ respectively. And coils $\mathrm{A}_{3}$ and $\mathrm{A}_{4}$, which contained the textile fibers, released $670( \pm 359.9)$ and $993( \pm 311.8) \mu \mathrm{g}$ respectively. It shows that using the polymer rod, the capacity could be increased by a factor 10 however using the fibers, the capacity was increased for a factor 18 , both compared to a coil with an empty interior.

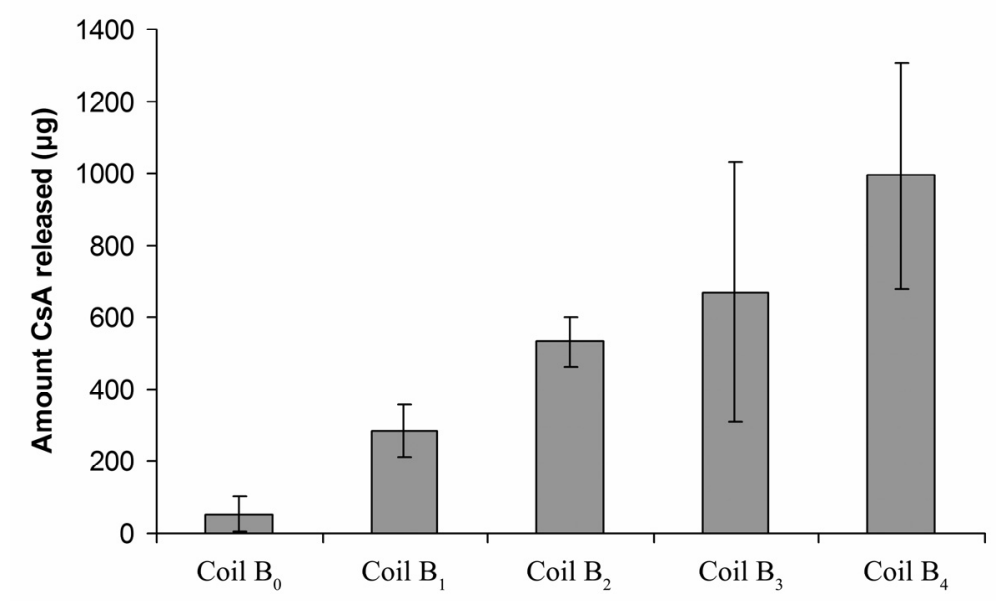

Figure 7.4. The amount of cyclosporine A released from different types of devices $(n=3)$.

The release experiment was also done in a system with a flow of $0.01 \mathrm{~mL} / \mathrm{min}$. Again, the windings of the coils were expanded (about $33 \%$ of its original size) to make sure the drug is released from the interior of the coil. Figure 7.5 shows the release profile of CsA from the coils 
$B_{2}$ and $B_{4}$ in time $(n=3)$. Because of expansion of the windings, the drug release starts immediately. The concentration decreases gradually over time for at least 72 hours. As expected, coil $\mathrm{B}_{4}$ released more amount of $\mathrm{Cs} A$, and the standard deviation of coil $\mathrm{B}_{4}$ was higher compared to coil $\mathrm{B}_{2}$.

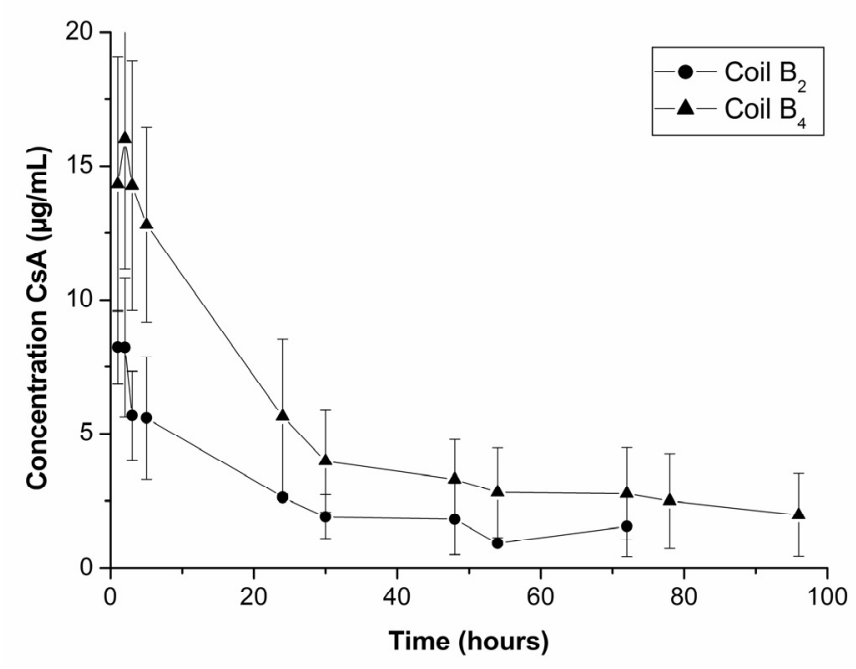

Figure 7.5. The release profile of cyclosporine $A$ in time from coil $B_{2}$ and $B_{4}(n=3)$.

\subsubsection{In vivo experiments}

\subsubsection{Mydriasis in dogs}

All coils could be inserted easily into the eyes of the Beagle dogs without the use of local or general anaesthetics. The animals showed no reaction of defense or discomfort at all. The tolerability of the device was excellent. Table 7.2 lists the average results of the parameters that were monitored during the study $(\mathrm{n}=8)$. After insertion of the coils into the eyes of the dogs, a delayed response to light was observed in two dogs already after 30 minutes. The rest of the animals showed a clear effect after one hour. The grade of pupil response to light was variable differing between delayed and completely no pupillar reaction.

As already found in previous studies with the OphthaCoil, the persistence in the lower conjunctival fornix can be quite variable. In this study, only two dogs kept the device in situ for the anticipated 6 hours. In all other animals, the coils were lost spontaneously before that time. It has already been suspected before that the third eyelid, an anatomical structure in nearly all 
animal species, except humans, may be the reason for the premature loss of the devices [23]. In spite of this, the mydriatic effect of phenylephrine and tropicamide was clearly visible in all animals. Onset of the reaction was visible after 30 minutes up to 1 hour. The effects lasted for around $1-4$ hours after the coils have been lost or removed. Figure 7.6 shows a photograph of the left eye of the same dog, before application of the device, and after 2.5 hours of wear, when the pupil was dilated. In none of the cases, a mydriatic effect has been observed on the contralateral eye, suggesting a strictly local and no systemic activity of the drugs.

Table 7.2 .The average results of the parameters monitored during the in vivo study. The response to light $(1=$ normal response, 2 = delayed response, 3 = no reaction to light $) ;$ and the extend of the mydriatic effect ( 1 = no dilation, 2 = moderate dilation, 3 = full dilation of the pupil) of the left eye of the dogs before and after placement of coil $C(n=8)$.

\begin{tabular}{|c|c|c|c|c|}
\hline \multirow[t]{2}{*}{ Time (hours) } & \multicolumn{2}{|c|}{ Presence coil } & \multirow{2}{*}{$\begin{array}{l}\text { Response to light } \\
\text { (SD) }\end{array}$} & \multirow{2}{*}{$\begin{array}{l}\text { Pupil dilation } \\
\text { (SD) }\end{array}$} \\
\hline & yes & no & & \\
\hline 0 & 0 & 8 & $1.00(0.00)$ & $1.00(0.00)$ \\
\hline Application & 8 & 0 & - & - \\
\hline 0.5 & 8 & 0 & $1.25(0.46)$ & $1.38(0.52)$ \\
\hline 1 & 8 & 0 & $2.63(0.52)$ & $2.63(0.52)$ \\
\hline 2 & 7 & 1 & $3.00(0.00)$ & $3.00(0.00)$ \\
\hline 3 & 5 & 3 & $2.88(0.35)$ & $2.63(0.52)$ \\
\hline 4 & 4 & 4 & $2.75(0.46)$ & $2.63(0.52)$ \\
\hline 5 & 2 & 6 & $2.63(0.52)$ & $2.75(0.46)$ \\
\hline 6 & 2 & 6 & $1.75(0.46)$ & $2.00(0.00)$ \\
\hline Removal & 0 & 8 & - & - \\
\hline 7 & 0 & 8 & $1.50(0.53)$ & $1.50(0.53)$ \\
\hline 24 & 0 & 8 & $1.00(0.00)$ & $1.00(0.00)$ \\
\hline
\end{tabular}



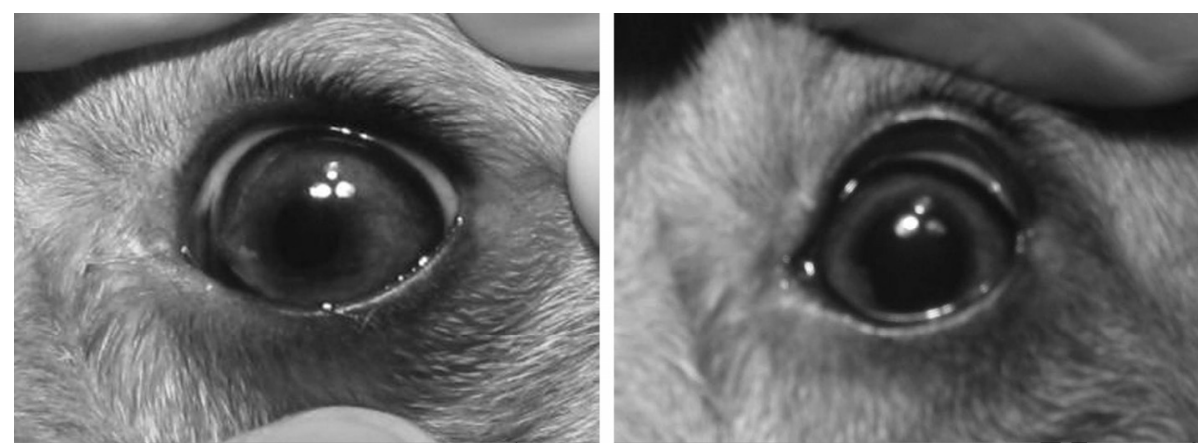

Figure 7.6. The canine eye before (A) and 2.5 hours (B) after placement of coil C.

\subsubsection{Tolerance evaluation in horses}

Insertion of the OphthaCoils was uneventful and easy, without the use of local anaesthetics. None of the horses had to be restrained or did show any avoidance behaviour or adverse reaction to the coil. Persistence in the conjunctival fornix was monitored around 3, 5 and 24 hours after insertion of the device. After 3 and 5 hours, all coils were still in place. Obviously both coils were lost over night and 24 hours after application, none of them could be recovered. Again, we hypothesize that the third eyelid may be the reason for the premature loss. Systemic or local reactions to the device could not be detected in both participating animals.

The delivery of cyclosporine A to the equine eye, for the treatment of uveitis, is a well known problem. The administration of medication to a horse at frequencies recommended for the treatment of corneal ulcers is often not feasible due to a lack of patient compliance [27]. In horses, it is almost impossible to retract the upper eyelid or tilt the head of the horse to apply an eye drop [28]. For the intense treatment of inflammation, ocular devices must be used. Examples of devices are a suprachoroidal implant, placed under general anaesthesia [29], and a subconjunctivally implanted, single-use, continuous-delivery pump [30]. The latter is attached to a subpalpebral lavage for continuous delivery of topical ophthalmic medications. The device continuously releases fluid at a predetermined rate of $0.02-4.0 \mathrm{~mL} / \mathrm{h}$ for up to 7 days. However, this method of drug delivery did not result in a stable concentration of medication in the tear film [27].

Based on the results of this study, the OphthaCoil may be suited to release drugs to the equine eye for at least 5 hours. Further studies on a larger number of horses may be necessary, as well as concentration measurements of the drug in the tear film. Fixation of the device using a bioresorbable suture, for example poly(lactic acid), may be useful for long term drug release. 
Then, the horse needs to be sedated only once for the placement of the device. The device will be lost from the eye after dissolution of the suture.

\subsection{Concluding remarks}

The animal model studies in this work show that the OphthaCoil provides a versatile platform for controlled release of drugs to the tear film of the eye. The new data reveal that the OphthaCoil could also play a role in veterinary medicine. The small version of the OphthaCoil is well-tolerated in the Beagle's eye, and may be useful in the combat of corneal infections. The large version of the OphthaCoil is most likely well acceptable for use in the horse eye. Moreover, different methods are considered to utilize the interior of both the small and the large OphthaCoils, although further technical work is required to improve the control over the drug release kinetics.

\section{References}

1. Davies N.M. Biopharmaceutical considerations in topical ocular drug delivery. Clin Exp Pharmacol Physiol 27 (7) (2000) 558-562.

2. Kaur I.P. and Kanwar M. Ocular preparations: the formulation approach. Drug Dev Ind Pharm 28 (5) (2002) 473-493.

3. Baudouin C. Allergic reaction to topical eyedrops. Curr Opin Allergy Clin Immunol 5 (5) (2005) 459-463.

4. Ghate D. and Edelhauser H.F. Ocular drug delivery. Expert Opin Drug Deliv 3 (2) (2006) 275-287.

5. Barbu E., Sarvaiya I., Green K.L., Nevell T.G. and Tsibouklis J. Vinylpyrrolidone-co-(meth)acrylic acid inserts for ocular drug delivery: Synthesis and evaluation. J Biomed Mater Res A 74 (4) (2005) 598-606.

6. Shargel L., Wu-Pong S. and Yu A.B. Applied Biopharmaceutics \& Pharmacokinetics, 5th edn. New York: McGray-Hill; 2005.

7. Weyenberg W., Vermeire A., Remon J.P. and Ludwig A. Characterization and in vivo evaluation of ocular bioadhesive minitablets compressed at different forces. J Control Release 89 (2) (2003) 329340.

8. Ceulemans J., Vermeire A., Adriaens E., Remon J.P. and Ludwig A. Evaluation of a mucoadhesive tablet for ocular use. J Control Release 77 (3) (2001) 333-344.

9. Hornof M., Weyenberg W., Ludwig A. and Bernkop-Schnurch A. Mucoadhesive ocular insert based on thiolated poly(acrylic acid): development and in vivo evaluation in humans. J Control Release 89 (3) (2003) 419-428.

10. Gurtler F., Kaltsatos V., Boisrame B., Deleforge J., Gex-Fabry M., Balant L.P. and Gurny R. Ocular availability of gentamicin in small animals after topical administration of a conventional eye drop solution and a novel long acting bioadhesive ophthalmic drug insert. Pharm Res 12 (11) (1995) 17911795 . 
11. Lv F.F., Li N., Zheng L.Q. and Tung C.H. Studies on the stability of the chloramphenicol in the microemulsion free of alcohols. Eur J Pharm Biopharm 62 (3) (2006) 288-294.

12. Medicines Evaluation Board, http://www.cbg-meb.nl (2007).

13. Alfonso E.C., Miller D., Cantu-Dibildox J., O'Brien TP. and Schein O.D. Fungal keratitis associated with non-therapeutic soft contact lenses. Am J Ophthalmol 142 (1) (2006) 154-155.

14. Chang D.C., Grant G.B., O'Donnell K., Wannemuehler K.A., Noble-Wang J., Rao C.Y., Jacobson L.M., Crowell C.S., Sneed R.S., Lewis F.M. et al Multistate outbreak of Fusarium keratitis associated with use of a contact lens solution. JAMA 296 (8) (2006) 953-963.

15. Iyer S.A., Tuli S.S. and Wagoner R.C. Fungal keratitis: emerging trends and treatment outcomes. Eye Contact Lens 32 (6) (2006) 267-271.

16. Bielory L. Ocular allergy and dry eye syndrome. Curr Opin Allergy Clin Immunol 4 (5) (2004) 421424.

17. Sall K., Stevenson O.D., Mundorf T.K. and Reis B.L. Two multicenter, randomized studies of the efficacy and safety of cyclosporine ophthalmic emulsion in moderate to severe dry eye disease. CsA Phase 3 Study Group. Ophthalmology 107 (4) (2000) 631-639.

18. Pflugfelder S.C. Antiinflammatory therapy for dry eye. Am J Ophthalmol 137 (2) (2004) 337-342.

19. Wilson S.E. and Perry H.D. Long-term resolution of chronic dry eye symptoms and signs after topical cyclosporine treatment. Ophthalmology 114 (1) (2007) 76-79.

20. Hospital statistics the Netherlands, http://www.prismant.nl (2007).

21. Pijls R.T., Cruysberg L.P., Nuijts R.M., Dias A.A. and Koole L.H. Capacity and tolerance of a new device for ocular drug delivery. Int J Pharm 341 (1-2) (2007) 152-161.

22. Pijls R.T., Lindemann S., Nuijts R.M., Daube G.W. and Koole L.H. Pradofloxacin release from the OphthaCoil: a new device for sustained delivery of drugs to the eye. J Drug Deliv Sci Tec 17 (1) (2007) 87-91.

23. Pijls R.T., Sonderkamp T., Daube G.W., Krebber R., Hanssen H.H., Nuijts R.M. and Koole L.H. Studies on a new device for drug delivery to the eye. Eur J Pharm Biopharm 59 (2) (2005) 283-288.

24. Aldenhoff Y.B., Knetsch M.L., Hanssen J.H., Lindhout T., Wielders S.J. and Koole L.H. Coils and tubes releasing heparin. Studies on a new vascular graft prototype. Biomaterials 25 (16) (2004) 31253133 .

25. Hanssen H.H., Wetzels G.M., Benzina A., van der Veen F.H., Lindhout T. and Koole L.H. Metallic wires with an adherent lubricious and blood-compatible polymeric coating and their use in the manufacture of novel slippery-when-wet guide wires: possible applications related to controlled local drug delivery. J Biomed Mater Res 48 (6) (1999) 820-828.

26. Lin H.R., Yu S.P., Kuo C.J., Kao H.J., Lo Y.L. and Lin Y.J. Pilocarpine-loaded chitosan-PAA nanosuspension for ophthalmic delivery. J Biomater Sci Polym Ed 18 (2) (2007) 205-221.

27. Myrna K.E. and Herring I.P. Constant rate infusion for topical ocular delivery in horses: a pilot study. Vet Ophthalmol 9 (1) (2006) 1-5.

28. Baeyens V.V., Percicot C., Zignani M., Deshpande A.A., Kaltsatos V.V. and Gurny R. Ocular drug delivery in veterinary medicine. Adv Drug Deliv Rev 28 (3) (1997) 335-361.

29. Gilger B.C. Equine Ophthalmology: Elsevier Saunders; 2005.

30. Blair M.J., Gionfriddo J.R., Polazzi L.M., Sojka J.E., Pfaff A.M. and Bingaman D.P. Subconjunctivally implanted micro-osmotic pumps for continuous ocular treatment in horses. Am J Vet Res 60 (9) (1999) 1102-1105. 


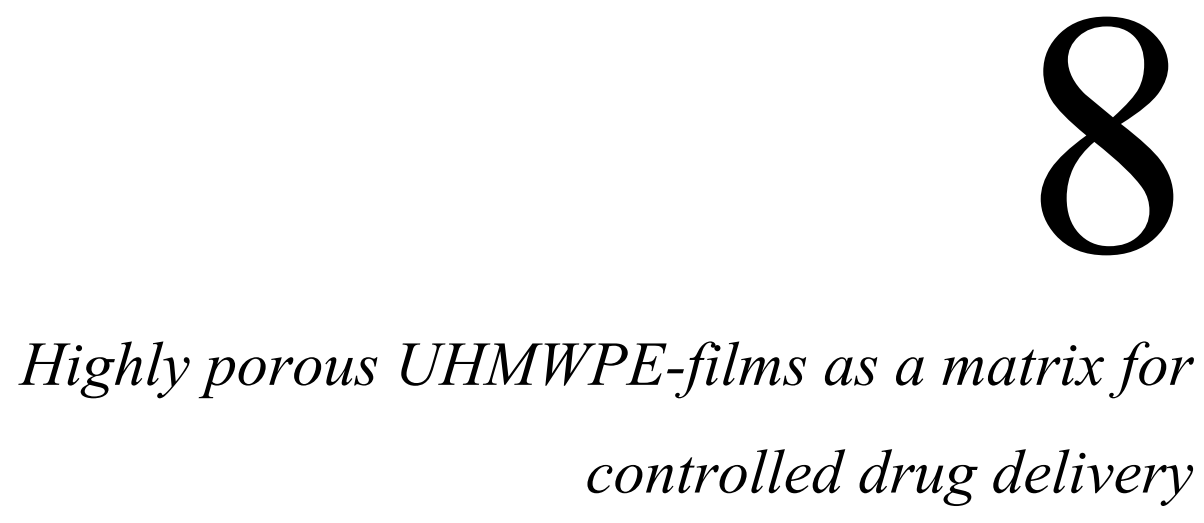

Rachel T. Pijls, Jérôme G.J.L. Lebouille, and Aylvin A. Dias, to be submitted

The work described in this manuscript led to the filing of two patents: 'Porous matrix for delivery of a bioactive agent' and 'Method for loading a bioactive agent in a porous matrix', both were filed on July 23, 2007 


\section{Abstract}

The use of a porous material for the controlled delivery of drugs was evaluated. Solupor ${ }^{\circledR}$, made from UHMWPE, was used as porous substrate. The porosity, thickness and pore size of the substrate could be varied. Drugs could be incorporated via precipitation of the drug, application of drug-loaded coating/impregnated layer(s), or a combination of both.

The antibiotic chloramphenicol was dissolved in ethanol and precipitated in the pores of Solupor $^{\circledR}$ with n-pentane. The release profile showed a small burst release for the first hour, followed by sustained release. However, the release rate was very slow; $19 \%$ was released after 19 days.

Application of drug-loaded coating layer(s) was achieved using an anilox coater. The pore size of the substrate plays an important role. Using pore sizes $\leq 0.1 \mu \mathrm{m}$, the substrate could be filled completely with the coating layer. Different pore sizes also influenced the drug release profile. Release from coated substrates with large pores $(>0.1 \mu \mathrm{m})$ showed more burst release than coated substrates with smaller pore sizes. By using multiple coating layers, the release could be tuned as well. Using a topcoat without a drug present, the burst release could be decreased. The combination of drug-precipitation and (drug-loaded) coating layer(s) resulted in a sustained release of $450 \mu \mathrm{g}$ of chloramphenicol for over 25 hours.

This approach could be used as a matrix for controlled drug delivery, for example in ophthalmology. A flat, drug-loaded substrate could be placed in the lower conjunctival fornix for topical drug delivery, whereas a rolled, tubular, drug-loaded substrate could be implanted, for example subscleral, for posterior drug release. 


\subsection{Introduction}

Controlled drug release materials have potential for utilization in biomedical implants, tissue engineering, and targeted drug delivery devices $[1,2]$. The advantages of controlled release include greater drug efficacy, better balanced drug concentrations in the body, and more comfort to the patient. In addition, local delivery of the drug reduces potential toxicity.

Porous materials offer a means to load such drugs and potentially exercise control over the release [1]. Examples of porous materials are nano- / microparticles [3, 4], scaffolds [5, 6] and membranes [7, 8]. However, rapid release, especially an initial burst release, has been observed in some cases. Charnay et al. reported that ibuprofen was released only during the initial 60 min from porous silica material, MCM 41 [9].

Here, a novel drug delivery device, based on a porous substrate, is described. The porous substrate is a film made from Ultra High Molecular Weight Polyethylene (UHMWPE), called Solupor $^{\circledR}$ (DSM Solutech, Geleen, the Netherlands). UHMWPE is one of the most common synthetic polymer that is used as an implant material. UHMWPE is currently used in 1.4 million patients around the world every year for use in the hip, knee, upper extremities and spine [10]. The UHMWPE is biaxially stretched in the presence of an organic solvent to generate a highly porous membrane with high gas, air and liquid permeability [11]. It has a unique combination of mechanical strength and high porosity. The porosity and thickness of the membrane can be varied via the PE recipe and biaxial stretching conditions. This results in film thicknesses ranging from $16 \mu \mathrm{m}$ to $120 \mu \mathrm{m}$, and porosity ranges of $36-50 \%$ and $75-85 \%$. The porosity was determined from the measured density and the density of the polyolefin bulk material [11]. A cross-section of the porous film, obtained by cryogenic fracturing, is shown in Figure 8.2A.

Solupor $^{\mathbb{B}}$ is used as a microporous membrane for various applications, like batteries, fuel cells and transdermal drug delivery systems $[12,13]$. In the transdermal drug delivery system (TDDS) Solupor ${ }^{\circledR}$ is used as a passive backing layer to deliver drugs. In this case, the skin is the rate-limiting factor in the drug release. Solupor ${ }^{\circledR}$ was tested for cytotoxicity, as well as on biocompatibility for skin contact, and it was FDA approved for this application.

Since Solupor ${ }^{\circledR}$ has good chemical resistance and is inert, tough, lightweight, and has high abrasion, impact resistance, and high gas, air and liquid permeability, it might be used for other biomedical applications than a TDDS. The other potential uses in the medical arena invariably tend to be more invasive than for transdermal applications. Solupor ${ }^{\circledR}$ could be used in various 
applications, like ocular drug delivery, an intravascular plug, mucosal drug delivery and drug delivery to the ear.

Our aim is to develop a drug delivery device with a release profile of several hours to several weeks, depending on the application. Solupor ${ }^{\mathbb{B}}$ can be loaded with a drug by precipitation of the drug in the pores and/or by impregnation of a drug-containing formulation, for example by photopolymerization. There are several advantages to using the photopolymerization technique in biomaterials [14-19]. In general, the process is benign and polymers can be fabricated at temperatures and $\mathrm{pH}$ values near physiological ranges and even in the presence of biologically active materials. The process also proceeds very rapidly at these conditions for most materials. Highly cross-linked polymer networks can be produced quasi instantly at ambient temperature by photoinitiated polymerization of multifunctional monomers and oligomers. UV-cured coatings are usually made of acrylate-based resins, known for their high reactivity, which undergo a radical-type polymerization upon UV exposure in the presence of a photoinitiator [20].

In considering photopolymerization, care has to be taken to consider two aspects related to the photochemical event: (i) the choice of wavelengths ranging from UV to visible light with respect to the drugs of choice and whether the irradiation is conducted in presence of biological tissue; (ii) the choice of photoinitiator in terms of biocompatibility and effect on biological tissues or drugs [21].

Here, the method for entrapping the drug is to polymerize a photopolymer in the presence of the drug in order to form a network around the solute. Drug diffusion out of the system occurs when the photopolymer is placed in an environment causing it to swell and the molecular mesh size to increase [16]. Photopolymers are considered as good candidate materials for many medical applications, e.g. contact lenses and dental cements. These photopolymers are now being considered for drug delivery applications where their polarity is tuned to the polarity of the drug [18]. For example, for hydrophilic drugs hydrogel photopolymers are used. Hydrogels can be used in many biomedical applications, including tissue engineering and drug delivery, because of their high water content, transport properties, and tissue-like physical and mechanical behavior [15]. However, before using this technique, the drug stability must be investigated to ensure that it can withstand the polymerization and will not react with the monomers in the system. 


\subsection{Materials and methods}

All chemicals were purchased from Sigma-Aldrich (Steinheim, Germany), unless stated otherwise.

\subsubsection{The porous substrate}

The different porous substrates were kindly provided by DSM Solutech (Geleen, the Netherlands). The names used to describe the different types of Solupor ${ }^{\mathbb{R}}$ reveal some properties of the substrate. For instance, Solupor ${ }^{\circledR} 10 \mathrm{P} 05 \mathrm{~A}$ has a total weight per surface area of $\underline{10} \mathrm{~g} / \mathrm{m}^{2}$, and a mean flow pore size of $0.5 \mu \mathrm{m}$. The first letter in the code stands for porous and hydrophobic (P) and the last letter (A) for the recipe. Table 8.1 lists the types of substrates used in this study and their properties.

\begin{tabular}{|c|c|c|c|c|c|c|}
\hline $\begin{array}{l}8 \\
8 \\
0\end{array}$ & $\begin{array}{l}\Theta_{\overline{0}} \\
\stackrel{0}{\Xi} \\
\stackrel{0}{\circ} \\
\mathscr{n}\end{array}$ & 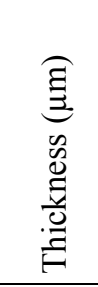 & 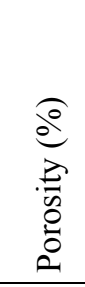 & 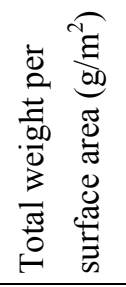 & 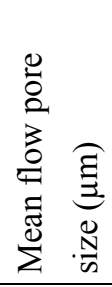 & 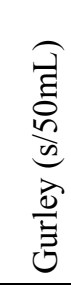 \\
\hline A & 7P07C & 45 & 84 & 7 & 0.7 & 3 \\
\hline B & 10P05A & 60 & 83 & 10 & 0.5 & 3 \\
\hline $\mathrm{C}$ & E-9P07A & $85-90$ & \pm 84 & 9 & 0.7 & n.a. \\
\hline $\mathrm{D}$ & $16 \mathrm{P} 05 \mathrm{~A}$ & 115 & 85 & 16 & 0.5 & 5 \\
\hline $\mathrm{E}$ & Y092C-8P01E & 16 & 42 & 8 & 0.1 & 160 \\
\hline $\mathrm{F}$ & 3P07A & 20 & 83 & 3 & 0.7 & 1.4 \\
\hline $\mathrm{G}$ & E115-P090628A & 19 & 36 & 12 & $<0.1$ & 290 \\
\hline $\mathrm{H}$ & 14P01E & 25 & 40 & 14.5 & 0.1 & 240 \\
\hline
\end{tabular}

\subsubsection{The active agents}

The pharmaceutical ingredients used for this study are fluorescein sodium (Fl-Na) and chloramphenicol (CAP), see Figure 8.1. Fl-Na is a commonly used dye in assessing tear turnover and corneal permeability [22]. The antibiotic CAP inhibits bacterial protein synthesis 
by blocking the peptidyl transferase step. It is an effective drug to treat eye infections and is often administered via eye drops [23, 24].

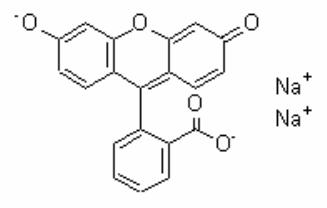

Fluorescein sodium

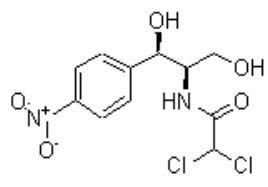

Chloramphenicol

Figure 8.1. The chemical structures of the active agents.

\subsubsection{Drug loading}

\subsubsection{Precipitation of a drug into the porous substrate}

A heated $\left( \pm 50^{\circ} \mathrm{C}\right)$ solution of $1.5 \mathrm{~g} \mathrm{CAP}$ in $2.0 \mathrm{~mL}$ EtOH was used. A weighed Solupor ${ }^{\circledR}$, with known surface area, was soaked into this hot solution. After wetting, the Solupor ${ }^{\mathbb{B}}$ was removed and washed directly in cold n-pentane. The cold n-pentane displaced the ethanol and CAP precipitated into and onto the Solupor ${ }^{\circledR}$ substrate. N-pentane was evaporated in air and the CAP crystals on the Solupor ${ }^{\circledR}$ were wiped off with a tissue soaked in n-pentane. The samples were checked by microscopy to ensure no residual CAP on the surface of the Solupor ${ }^{\mathbb{R}}$.

Three types of Solupor ${ }^{\circledR}$ with different pore sizes were used to study the result and reproducibility of the impregnation process. The types used were type B (10P05A), G (E115P090628A), and H (14P01E). The substrates were weighed before and after this procedure to calculate the amount of drug in the pores.

As control experiment to check the integrity of the Solupor ${ }^{\circledR}$ substrate after this procedure, the substrate underwent the same impregnation process as described above, without the use of CAP. The thickness and the laminated structure of the Solupor ${ }^{\circledR}$ were examined using scanning electron microscopy (SEM).

\subsubsection{Coating procedure and photopolymerization}

The second method used for drug loading of Solupor ${ }^{\circledR}$, was using a coating procedure. The drug matrix (formulation) used was a poly(2-methyl-1,4-butanediol)-alt-poly(tetramethylene glycol)-based urethane acrylate oligomer (PTGL, [25]). This oligomer is already used as primer coating in lubricious coatings on catheters. It passed the tests for cytotoxicity (ISO 10993-5) and irritation and sensitization (ISO 10993-10). The monomer hexanediol diacrylate 
(HDDA) was added to the oligomer in order to create a denser network to facilitate drug entrapment, as well as to increase the drug solubility in the formulation. Two different ratios were used: 70/30 and 30/70 w/w ratio of PTGL/HDDA. Furthermore, the solvent ethanol, the photoinitiator Irgacure 2959 (2.0 $\mathrm{w} \%$ of the solids in the formulation), and the stabilizer Irganox $1035(0.1 \mathrm{w} \%$ of the solids, Ciba Specialty Chemicals Inc., Groot-Bijgaarden, Belgium) were used. The photoinitiator used is well tolerated over a wide range of cell types and chemical concentrations [26]. A summary of the different drug-containing formulations used, are given in Table 8.2. Formulations containing the dye Fl-Na (I) or the drug CAP (II-IV) were prepared, where the dye/drug replaced the PTGL and HDDA in the given ratio. In addition two formulations were prepared without a drug (V and VI).

Table 8.2. The components of the formulations used for coating the Solupor ${ }^{\circledR}$.

\begin{tabular}{ccccccc}
\hline Formulation & $\begin{array}{c}\text { Ethanol } \\
(\mathrm{w} \%)\end{array}$ & $\begin{array}{c}\text { PTGL } \\
(\mathrm{w} \%)\end{array}$ & $\begin{array}{c}\text { HDDA } \\
(\mathrm{w} \%)\end{array}$ & $\begin{array}{c}\text { Irgacure } \\
2959(\mathrm{w} \%)\end{array}$ & $\begin{array}{c}\text { Irganox } \\
1035(\mathrm{w} \%)\end{array}$ & $\begin{array}{c}\text { Dye / Drug } \\
\left.\mathrm{w} \% \text { (w\% of solids }^{*}\right)\end{array}$ \\
\hline I & 40 & 36.92 & 15.82 & 1.20 & 0.06 & $6.0(10.0) \mathrm{Fl}-\mathrm{Na}$ \\
II & 40 & 36.92 & 15.82 & 1.20 & 0.06 & $6.0(10.0) \mathrm{CAP}$ \\
III & 70 & 11.39 & 4.89 & 0.40 & 0.02 & $13.3(43.5) \mathrm{CAP}$ \\
IV & 40 & 15.31 & 35.73 & 1.20 & 0.06 & $7.7(12.7) \mathrm{CAP}$ \\
V & 40 & 17.59 & 41.05 & 1.30 & 0.06 & - \\
VI & 40 & 41.12 & 17.62 & 1.20 & 0.06 & - \\
\hline
\end{tabular}

${ }^{*}$ The number in the brackets represent the weight percentage of drug after the polymerization, thus after evaporation of the solvent ethanol.

These formulations were applied onto the Solupor ${ }^{\circledR}$ substrate using an Esiproof anilox coater (Erichsen, Saint-Antoine, France). An anilox coater is a precision instrument for accurate application of coatings onto a surface. The Esiproof consists of a precision-engineered unit into which the anilox roller, EPDM stereo roller and doctor blade are easily positioned. The substrate to be proofed is placed on a flat, hard surface. Several milliliters of the drug containing liquid formulation from a pipette are dropped into the ink reservoir of the Esiproof applicator, and the unit is then pulled over the substrate to produce a sample. Samples up to 1 meter long can be made.

The Solupor ${ }^{\circledR}$ substrate was taped onto a glass plate and the formulation was applied using the anilox coater. The formulation layer was polymerized using UV-light. The samples were prepared by curing coatings of $5-15 \mu \mathrm{m}$ thickness on glass plates at $20^{\circ} \mathrm{C}$, in a nitrogen 
atmosphere, on a conveyor belt, fitted with a Fusion F600 (6000W, Fusion UV Systems Inc., Gaithersburg, USA) electrodeless D-bulb. A UV dose of $1.0 \mathrm{~J} / \mathrm{cm}^{2}\left(>5.0 \mathrm{~W} / \mathrm{cm}^{2}\right)$ was measured using a UV Power Puck Light meter (EIT Inc., Virginia, USA). Then, the substrate was cut from the glass plate, turned and taped again to the glass plate. The application and the curing process of the formulation were repeated on the reverse side. The films were weighed before and after the dual side coating procedure to calculate the amount of drug in the coating. To investigate the effect of this coating procedure onto several types of Solupor ${ }^{\circledR}$, this process was done using formulations I and II for all the different types of Solupor ${ }^{\circledR}$ listed in Table 8.1.

\subsubsection{Multilayer application}

In the following experiments Solupor ${ }^{\circledR}$ type $\mathrm{H}, 14 \mathrm{P} 01 \mathrm{E}$ with pore size of $0.1 \mu \mathrm{m}$, and the drug CAP were used. The effects of multilayer application of the coating, as well as combinations of the loading techniques, on the drug release were investigated.

Here, different drug-containing formulations have been used, see Table 8.2. In the experiments, three effects on drug release were studied: (i) the effect of drug loading concentration; (ii) the effect of cross-link density; and (iii) the effect of multiple coating layers. Formulation III had an increased amount of CAP and ethanol. In formulation IV, the ratio of the PTGL-oligomer and HDDA was changed from $70 / 30$ to $30 / 70 \mathrm{w} / \mathrm{w} \%$ to create a denser network. Formulations $\mathrm{V}$ and VI did not contain the drug CAP to study the effect of a 'blank' top layer on the release profile. Then, the effect of multilayered coatings on the drug release was studied. Solupor ${ }^{\mathbb{B}}$ type $\mathrm{H}$ was coated with the following combinations: formulation III only, formulation III and IV, III and V, and formulation III and VI, see Table 8.6.

Finally, the combination of drug precipitation into the pores and (multilayered) coating of the substrate is investigated. Here, CAP was first precipitated into the pores of the Solupor ${ }^{\mathbb{P}}$ substrate, via the method described in section 8.2.3.1. Then, the drug-loaded substrate was coated using the anilox coater with formulation III only, and with the following combinations: formulations III and IV, and III and V, listed in Table 8.6 as well. Again, all films were weighed before and after curing to calculate the amount of drug in the coating.

\subsubsection{Characterization}

\subsubsection{FT-IR measurements}

The acrylate conversion of the formulations was studied using FT-IR spectrometry. A Perkin Elmer Spectrum One FTIR spectrometer equipped with a Golden Gate attenuated total 
reflection (ATR) accessory was used. The Spectrum One consists of a DTGS detector and the Golden Gate makes use of a single bounce diamond crystal. Infrared spectra between 4000 and $650 \mathrm{~cm}^{-1}$ were recorded averaging 20 scans with a spectral resolution of $4 \mathrm{~cm}^{-1}$. The transmission spectra were transformed in absorption spectra. The $2^{\text {nd }}$ derivative with 25 points Savitsky Golay was taken and the peak height was determined at a frequencies of 810 and 1410 $\mathrm{cm}^{-1}$, typical double bond frequencies for acrylate conversions [27, 28]. The acrylate conversion was calculated using Equation 8.1. Various formulation batches were used, see Table 8.3. All formulations contained $0.06 \mathrm{w} \%$ Irganox 1035, $1.2 \mathrm{w} \%$ Irgacure 2959, and were prepared in $40 \mathrm{w} \%$ ethanol.

$$
\text { Conversion }(\%)=\frac{I_{\text {ref }}-I_{\text {sample }}}{I_{\text {ref }}} * 100 \%
$$

Table 8.3. The components of the formulation batches

\begin{tabular}{ccc}
\hline Component & $\begin{array}{r}\text { PTGL/HDDA } \\
\text { (w/w\% ratio) }\end{array}$ & Drug / Dye \\
\hline Batch 1 & $70 / 30$ & - \\
Batch 2 & $70 / 30$ & $5 \% \mathrm{Fl}-\mathrm{Na}$ \\
Batch 3 & $70 / 30$ & $10 \% \mathrm{Fl}-\mathrm{Na}$ \\
Batch 4 & $30 / 70$ & $5 \% \mathrm{Fl}-\mathrm{Na}$ \\
Batch 5 & $70 / 30$ & $10 \% \mathrm{CAP}$ \\
\hline
\end{tabular}

\subsubsection{LM/SEM characterization}

Cross-sections of the impregnated and/or coated porous films were prepared by cryogenic fracturing. Cross-sections of the films containing Fl-Na could be investigated using a light microscope (LM). The Leica MZFLIII equipped with a CC12 camera and analysis software was used. Photos were made with reflected light and fluorescence light. Samples containing the drug CAP were investigated with scanning electron microscopy (SEM). The specimens were mounted in a SEM sample holder and a conductive Au-layer was applied (2x60s, $20 \mathrm{~mA})$. Sample SEM imaging was done using a Philips CP SEM XL30 at accelerating voltages of 5 and $10 \mathrm{kV}$.

Energy Dispersive X-ray (EDX) Analysis was performed using a Phoenix EDAX-system. In the mapping, the chlorine atoms of the pharmaceutical agent CAP were highlighted, which may prove that the drug was incorporated into the Solupor ${ }^{\circledR}$. Different coated substrates were 
used to check the incorporation of the drug into the substrate. Table 8.4 lists the Solupor ${ }^{\mathbb{R}}$ substrates and their applied coatings used for the mappings.

Table 8.4. The substrates and their coatings used for the chlorine mapping

\begin{tabular}{cccc}
\hline Substrate & Precipitation of CAP & $1^{\text {st }}$ coating* & $2^{\text {nd }}$ coating* \\
\hline $\mathrm{H}$ & - & II & - \\
$\mathrm{H}$ & Yes & - & - \\
$\mathrm{H}$ & Yes & III & - \\
$\mathrm{H}$ & Yes & III & IV \\
\hline
\end{tabular}

*Codes of the coating refer to the formulations according to Table 2.

\subsubsection{Spectrophotometer}

The release of the dye Fl-Na could be detected using a spectrophotometer. A SpectraMax Gemini equipped with SoftMax Pro software was used. The absorption and emission wavelength of fluorescein sodium are $490 \mathrm{~nm}$ and $514 \mathrm{~nm}$ respectively [22, 29]. In a 96-wells plate, $100 \mu \mathrm{L}$ of every sample was injected in triplo, and using a dilution series the amount of fluorescein sodium released could be calculated.

\subsubsection{HPLC measurement}

High performance liquid chromatography (HPLC) was used to measure the concentrations of CAP in the samples. A Hewlett Packard HP 1090 HPLC apparatus equipped with a C18 analytical column 150 x $4.6 \mathrm{~mm}$ (X Terra RP 18, Waters, Eschborn, Germany) was used with a mean particle size of $3.5 \mu \mathrm{m}$. As eluents, a $10 \mathrm{mM}$ phosphoric acid / water solution (phase A) and acetonitrile (phase B) were used. The temperature was set at $40^{\circ} \mathrm{C}$ and the software used was ChemStation software version Rev. A. 08.03 (Agilent Technologies, Amstelveen, the Netherlands).

In the gradient cycle of 15 minutes, the mobile phase was changed from $10 \%$ to $70 \%$ of the mobile phase $\mathrm{B}$ over a period of 10 minutes; kept at $70 \%$ for 1 minute and, thereafter changed to $90 \%$ of the mobile phase B over a period of 0.1 minute; again kept at $90 \%$ for 1 minute, and lowered to $10 \%$ in 0.1 minute, where it was kept until the next sample was injected. A flow rate of $1.5 \mathrm{~mL} / \mathrm{min}$ and an injection volume of $150 \mu \mathrm{L}$ were used during the analysis. The detection was done at wavelengths of 200 and $280 \mathrm{~nm}$. The drug concentration in the samples could be calculated using a dilution series with samples of $0.09 \mu \mathrm{g} / \mathrm{mL}-45.0 \mu \mathrm{g} / \mathrm{mL}$ CAP in water. 


\subsubsection{Drug stability}

The effects of UV light and photo-generated radicals on the stability of the drug CAP were studied. This was done in three phases:

- In phase I, the effect of UV light on the stability of the drug was measured. The concentration of the drug in solution before and after UV irradiation was measured and compared. A drug solution of $5.0 \mathrm{mg}$ chloramphenicol in $50 \mathrm{~mL}$ water/ethanol $(1: 1 \mathrm{v} / \mathrm{v})$ was prepared. Three HPLC vials were filled with $1 \mathrm{~mL}$ of this drug containing solution. One vial was not exposed to UV light (reference), the second vial was exposed to 2.0 $\mathrm{J} / \mathrm{cm}^{2}$ and the third vial was exposed to $12.0 \mathrm{~J} / \mathrm{cm}^{2}$.

- In phase II, the additional effect of photogenerated radicals (through UV photodecomposition of a photoinitiator) on the drug was measured. Here, $5.0 \mathrm{mg}$ chloramphenicol and $5.0 \mathrm{mg}$ photoinitiator (Irgacure 2959) were dissolved in $50 \mathrm{~mL}$ water/ethanol $(1: 1 \mathrm{v} / \mathrm{v})$. Again, three HPLC vials were filled with $1 \mathrm{~mL}$ of this solution, and the same procedure was followed for the UV irradiation.

- Phase III examines the combined effect of UV light and radicals in the presence of an acrylate-type monomer on the stability of the drug. The monomer is present in a 100 -fold excess relative to the drug. The photogenerated radicals can react with both the drug and the unsaturated monomer. Now, $5.0 \mathrm{mg}$ chloramphenicol, $5.0 \mathrm{mg}$ photoinitiator and $0.5 \mathrm{~g}$ poly(propylene glycol) acrylate were dissolved in $50 \mathrm{~mL}$ water/ethanol $(1: 1 \mathrm{v} / \mathrm{v})$.

All samples were done in five-fold. UV irradiation of drug containing solutions was performed with a lab UV source Dr. Hoenle Bluepoint 2, equipped with a D-bulb, directed onto the (open) HPLC vials by a UV light guide. After exposure to the UV light, the vials were shaken to homogenize the samples, and the drug amount present was measured by HPLC, using the following conditions. As eluents, again a $10 \mathrm{mM}$ phosphoric acid / water solution (A) and acetonitrile (B) were used. In the gradient cycle of 10 minutes, the mobile phase was changed from $30 \%$ to $70 \%$ of the mobile phase B over a period of 5 minutes; kept at $70 \%$ for 1 minute and, thereafter changed to $90 \%$ of the mobile phase B over a period of 0.1 minute; again kept at $90 \%$ for 1 minute, and lowered to $30 \%$ in 0.1 minute, where it was kept until the next sample was injected. A flow rate of $1.5 \mathrm{~mL} / \mathrm{min}$, temperature of $40^{\circ} \mathrm{C}$, and an injection volume of $5 \mu \mathrm{L}$ were used during the analysis. The detection was done at the wavelength of 200 and $280 \mathrm{~nm}$. A dilution series of $0.4-0.025 \mathrm{mg} / \mathrm{mL}$ CAP in water/ethanol $(1 / 1 \mathrm{v} / \mathrm{v})$ was made to calculate the amount of drug in the samples. 


\subsubsection{Drug release}

The release of the dye or drug from the different drug-loaded (and coated) Solupor ${ }^{\circledR}$ substrates was measured in demineralized water at room temperature. Eppendorf vials were filled with $1.5 \mathrm{~mL}$ water and pieces of $1 \times 1 \mathrm{~cm}^{2}$ films were added. At different time points the films were removed from the vials, dried on a filter paper and introduced into vials with fresh demineralized water. Then, the samples were analyzed via spectrophotometry or HPLC, as described in section 8.2.4.

The treated Solupor ${ }^{\circledR}$ substrates that were measured are listed in the Tables 8.5 and 8.6. Table 8.5 lists all substrates single coated with formulation I or II, all measured in fourfold. Table 8.6 lists the substrates with multiple coating layers, and the substrates loaded with both methods: the CAP precipitated in the pores and coated afterwards, all measured in fivefold.

\subsection{Results and Discussion}

\subsubsection{Drug loading}

\subsubsection{Precipitation of the drug in the porous substrate}

After precipitation and drying in the air, the weight increase of the different impregnated Solupor $^{\circledR}$ substrates was measured. The tests were executed in triplo. The average weight increase of Solupor $^{\circledR}$ type B (pore size $0.5 \mu \mathrm{m}$ ), G (pore size $<0.1 \mu \mathrm{m}$ ), and H (pore size 0.1 $\mu \mathrm{m})$ were $180.03 \%$ (10.82), $24.65 \%$ (0.08), and $19.14 \%(0.21)$ respectively.

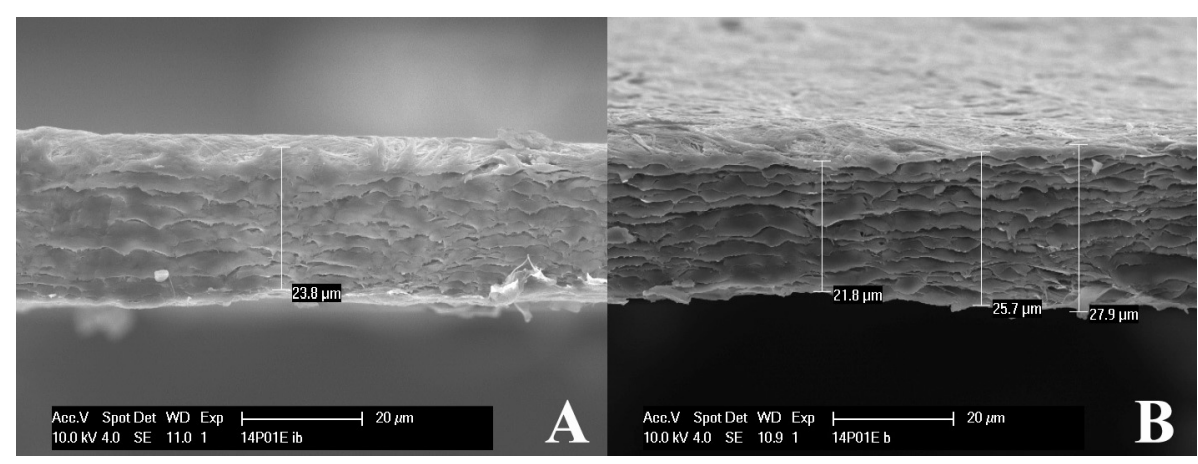

Figure 8.2. SEM photographs of an untreated Solupor ${ }^{\circledR}$ type $H(A)$, and Solupor ${ }^{\circledR}$ type H which underwent the precipitation process without using the drug $(B)$. 
The results show that this procedure is reproducible. The thicker the Solupor ${ }^{\mathbb{R}}$ and higher the porosity, the more drug that could be incorporated into the Solupor ${ }^{\circledR}$ (e.g. type B compared to type $\mathrm{H})$. The control experiment, where the loading procedure in the absence of drug was followed, showed no changes in the laminated structure or thickness of the substrate, see Figure 8.2.

\subsubsection{Coating procedure and photopolymerization}

For coating the Solupor ${ }^{\circledR}$ substrates, two formulations with different active agents were prepared. Formulation I contained the dye Fl-Na, which was dispersed in the formulation and formulation II contained the drug CAP, which could be dissolved.

The Solupor ${ }^{\circledR}$ types A, B, C and D were coated on both sides using formulation I, containing the dye Fl-Na. The substrates turned orange/red and were opaque. The other Solupor ${ }^{\circledR}$ types, which were thinner and had smaller pore sizes, could not be coated properly with this formulation. Applying the formulation onto the Solupor ${ }^{\circledR}$ substrate went well, but the substrate broke during the UV polymerization process, probably due to shrinkage. This is attributed to the fixation of Solupor ${ }^{\circledR}$ on the glass plate to ensure that it is flat on the UV curing rig. In addition, the fixation is required so that the light porous substrate does not get sucked into the cooling and extraction fan that maintains the temperature of the bulb. In an industrial conveyorized wet coating facility this can be avoided with adjustable tension settings.

Using formulation II, containing the drug CAP, all different Solupor ${ }^{\circledR}$ substrates could be coated successfully on both sides. Most substrates turned white/cream. The substrates with small pore sizes $(\leq 0.1 \mu \mathrm{m})$ became transparent. Table 8.5 shows the total amount of dye/drug estimated to be present in $1 \mathrm{~cm}^{2}$ of the coated substrate.

\begin{tabular}{ccc} 
Table 8.5. The total amount of dye/drug present in $1 \mathrm{~cm}^{2}$ coated substr \\
\hline Solupor $^{\circledR}$ & Fluorescein sodium & Chloramphenicol \\
\hline $\mathrm{A}$ & $133 \mu \mathrm{g}$ & $111 \mu \mathrm{g}$ \\
$\mathrm{B}$ & $136 \mu \mathrm{g}$ & $120 \mu \mathrm{g}$ \\
$\mathrm{C}$ & $144 \mu \mathrm{g}$ & $136 \mu \mathrm{g}$ \\
$\mathrm{D}$ & $159 \mu \mathrm{g}$ & $122 \mu \mathrm{g}$ \\
$\mathrm{E}$ & - & $64.5 \mu \mathrm{g}$ \\
$\mathrm{F}$ & - & $66.1 \mu \mathrm{g}$ \\
$\mathrm{G}$ & - & $65.2 \mu \mathrm{g}$ \\
$\mathrm{H}$ & - & $79 \mu \mathrm{g}$ \\
\hline
\end{tabular}




\subsubsection{Different formulations and multilayer application}

All combinations listed in Table 8.6, could be prepared successfully. The amount of CAP present in the prepared coated substrates (all type $\mathrm{H}$ ) is listed in the table as well.

Table 8.6. The total amount of drug present on the substrate using different coatings and loading

techniques.

\begin{tabular}{ccccc}
\hline Sample & $\begin{array}{c}\text { Precipitation of } \\
\text { drug into pores? }\end{array}$ & $1^{\text {st }}$ coating & $2^{\text {nd }}$ coating & $\begin{array}{c}\text { Total amount of CAP } \\
\left(\mu \mathrm{g} / \mathrm{cm}^{2}\right)\end{array}$ \\
\hline 1 & - & III & - & 201 \\
2 & - & III & IV & 218 \\
3 & - & III & V & 204 \\
4 & - & III & VI & 209 \\
\hline i.0 & yes & - & - & 268 \\
i.1 & yes & III & - & 476 \\
i.2 & yes & III & IV & 483 \\
i.3 & yes & III & V & 454 \\
\hline
\end{tabular}

\subsubsection{Characterization}

\subsubsection{FT-IR measurements}

For Batch 1, without a dye or drug, no residual acrylates could be detected. The detection limit was set at $3 \%$, which means that the acrylate conversion was over $97 \%$. The same results were found for Batch 2 and 4, which contained 5\% of the dye and drug, see Figure 8.3.

For batches 3 and 5, containing $10 \mathrm{w} \%$ of the dye or drug, the acrylate conversion was $81 \%$ $( \pm 10)$ and $82 \%( \pm 4)$ respectively. Especially the batch containing the Fl-Na had a large systematic error, which can be explained by the inhomogeneity of the coating formulation (dispersion). Even though CAP, which has an absorption maximum at $280 \mathrm{~nm}$ (similar to the absorption maximum of the photoinitiator), while Fl-Na has an absorption maximum at $490 \mathrm{~nm}$ (i.e. in a totally different range as the photoinitiator), surprisingly in both $5 \%$ and $10 \%$ of drug loading no significant differences in the acrylate conversion for these formulations were seen. In addition, the polymerization rates of batch 1 and 2 were measured using real-time FT-IR $(\mathrm{n}=2)$. The acrylate conversion after $60 \mathrm{~s}$ UV illumination $\left(85 \mathrm{~mW} / \mathrm{cm}^{2}\right)$ was plotted against the illumination time. The results showed that the addition of Fl-Na lowers the rate of 
polymerization significantly. The conversion rate of batch 1 was $3.911 \mathrm{~mol} / \mathrm{dm}^{3}\left(113 \% \cdot \mathrm{s}^{-1}\right)$, as the rate of batch 2 was $2.776 \mathrm{~mol} / \mathrm{dm}^{3}\left(83 \% \cdot \mathrm{s}^{-1}\right)$, assuming a density of $1 \mathrm{~g} / \mathrm{cm}^{3}$.

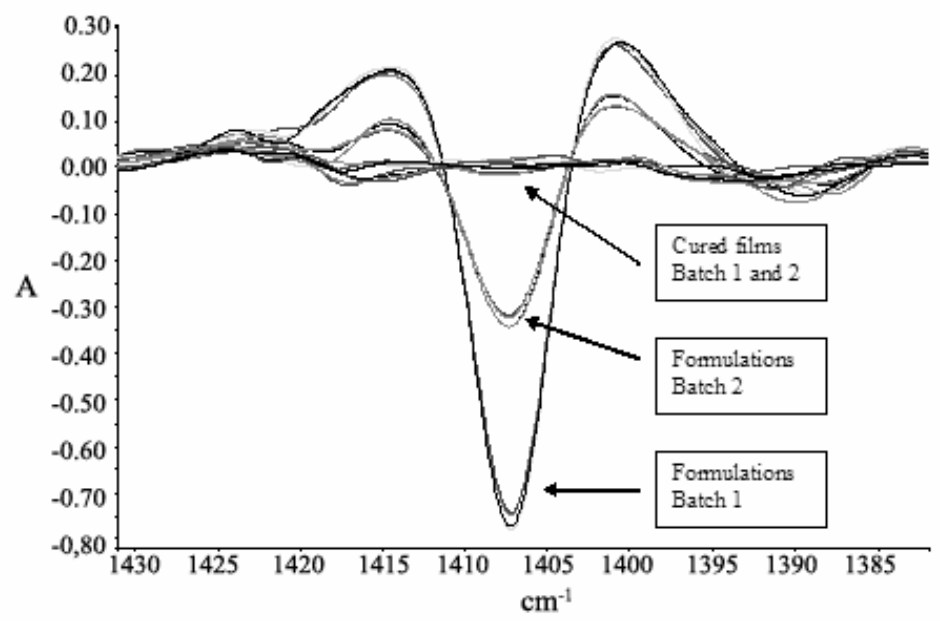

Figure 8.3. $2^{\text {nd }}$ derivative FTIR spectra enlarged in the spectral region characteristic for $\mathrm{C}-\mathrm{H}$ bend vibration $\left(1407 \mathrm{~cm}^{-1}\right)$ of an acrylate unsaturation.

\subsubsection{SEM/LM characterization}

Figure 8.4 shows the cross-section of different coated Solupor ${ }^{\circledR}$ substrates. Figure 8.4A shows a LM photograph of the Solupor ${ }^{\circledR}$ substrate coated with formulation I containing Fl-Na, using fluorescence light. The picture shows that the laminated structure of the substrate is lost and that clusters of Fl-Na were accumulated in the pores.

Figures 8.4B-D shows SEM photographs of cross-sections of different substrates coated with formulation II. It shows that more of the substrate was filled with the coating, as the pore sizes of the substrate were smaller.

EDX-mapping of the chlorine atoms of the different coated substrates, given in Table 8.5, was performed. Figure 8.5A-B shows that when the substrate (type $\mathrm{H}$ ) is only coated with a CAP containing formulation, the chlorine atoms are concentrated at the surface of the sample. When the substrate is impregnated first, and thereafter coated with formulation III and IV, the chlorine atoms are evenly distributed over the sample, see Figure 8.5C-D. 


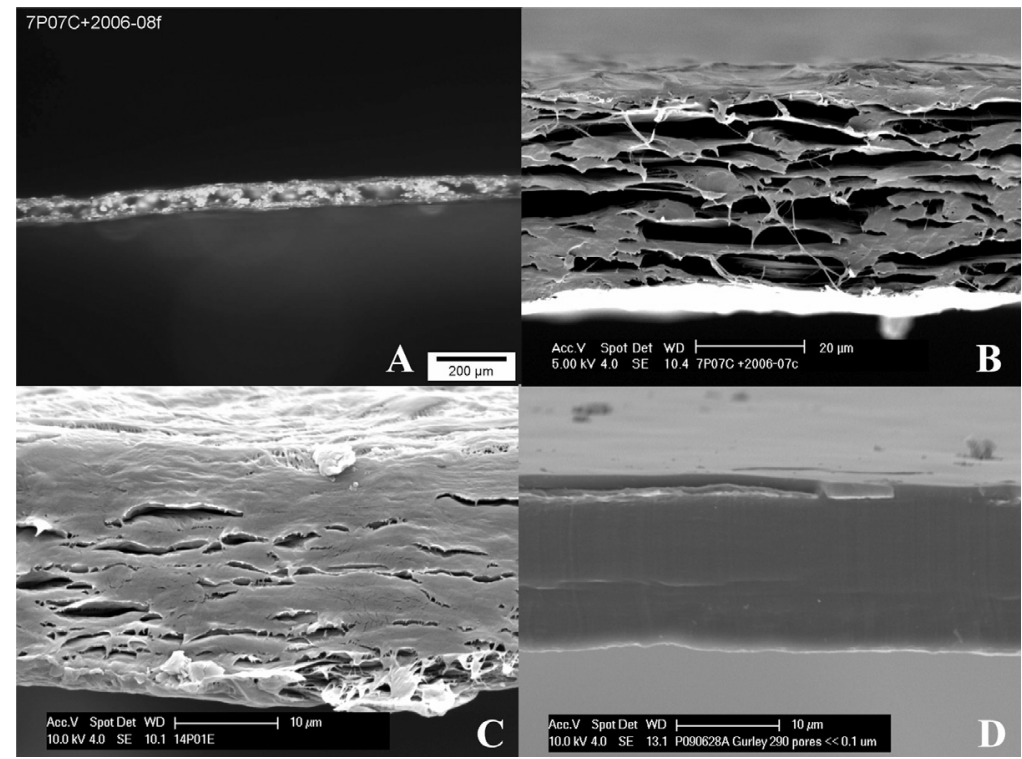

Figure 8.4. Cross-sections of coated Solupor ${ }^{\circledR}$ substrates. (A) LM photograph of a Fl-Na coated Solupor ${ }^{\circledR}$ type A (pore size $0.7 \mu \mathrm{m}$ ); SEM photograph of CAP coated Solupor ${ }^{\circledR}$, (B) type A (pore size $\left.0.7 \mu \mathrm{m}\right),(C)$ type $H$ (pore size $0.1 \mu \mathrm{m})$, and (D) type $G$ (pore size $<0.1 \mu \mathrm{m})$.

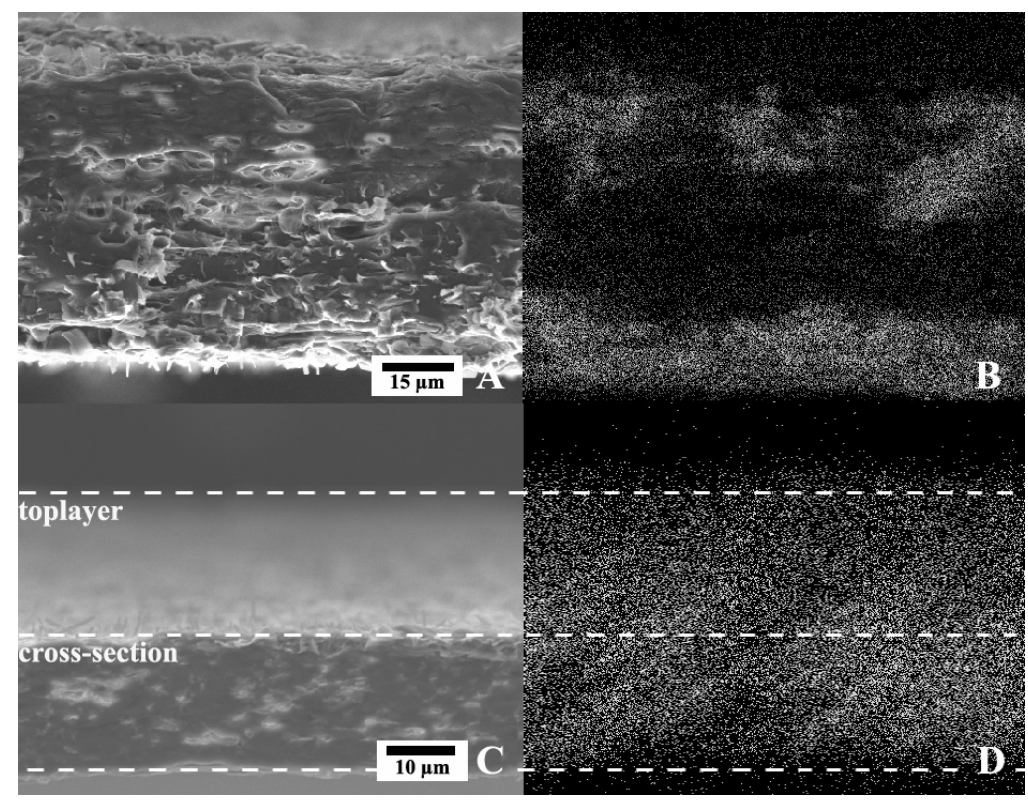

Figure 8.5. Mapping of chlorine atoms. (A) SEM photograph of coated Solupor ${ }^{\circledR}$, (B) Chlorine atoms in sample coated Solupor ${ }^{\circledR}$, (C) SEM photograph of impregnated and coated Solupor ${ }^{\circledR}$, and (D) Chlorine atoms in impregnated and coated Solupor ${ }^{\circledR}$. 


\subsubsection{Drug stability}

The effect of UV light and radicals on the stability of CAP in the presence and absence of an acrylate monomer was investigated. Figure 8.6 shows the different phases and the percentage recovery of the drug plotted against the irradiation dose. Phase I showed that CAP is not affected by UV light. The recovery is still $100 \%$, even at the high irradiation dose of $12 \mathrm{~J} / \mathrm{cm}^{2}$. In phase II, it is shown that CAP is affected by the photogenerated radicals of the photoinitiator, but only at the highest irradiation level of $12 \mathrm{~J} / \mathrm{cm}^{2}$. Here, the percentage of recovery was decreased to $90 \%$. Phase III showed that the presence of poly(propylene glycol) acrylate has a protecting effect on the drug at an irradiation level of $12 \mathrm{~J} / \mathrm{cm}^{2}$. Typical

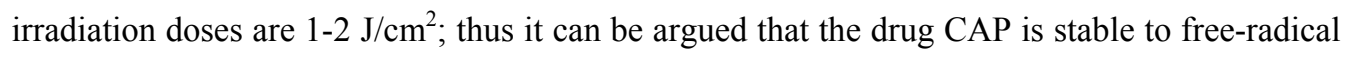
photo-initiated polymerization processes.

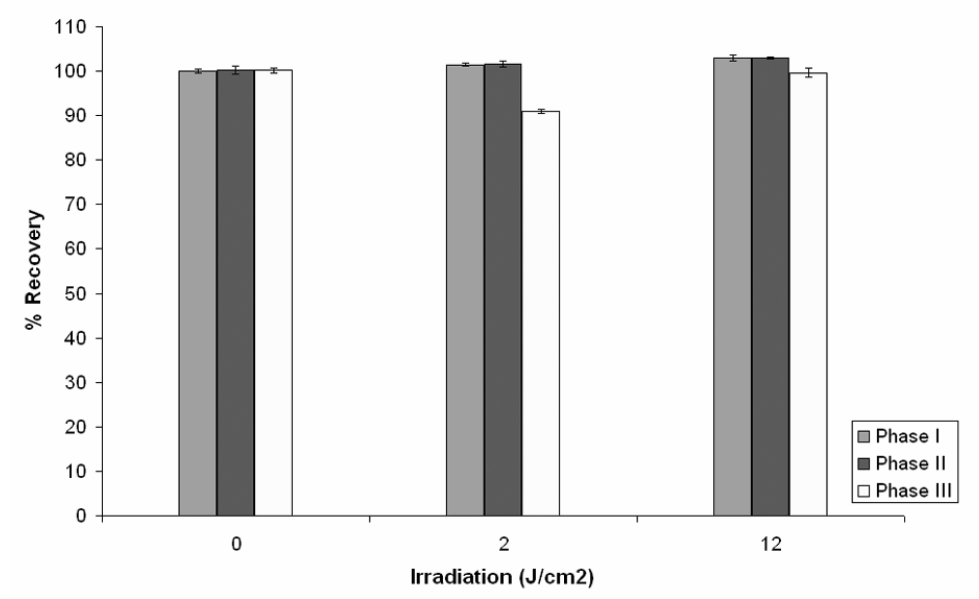

Figure 8.6. The effect of UV dose, initiating radicals and the monomer (poly(propylene glycol) acrylate) on the drug recovery.

\subsubsection{Drug release}

The Solupor ${ }^{\circledR}$ substrates A-D were coated with formulation I, which contained Fl-Na. The release of Fl-Na was characterized by a burst release, i.e. 75-85\% was released within the first few hours, followed by sustained release for almost 4 weeks $(n=4)$. Substrate type A and C released over $97 \%$ of their drug, whereas type $\mathrm{B}$ and $\mathrm{D}$ released $85-86 \%$. This may be due to the difference in pore size of the substrates, namely 0.7 and $0.5 \mu \mathrm{m}$ respectively.

The Solupor ${ }^{\circledR}$ substrates A-D coated with formulation II, containing the drug CAP, resulted in a release of $58-76 \%$ of their entrapped drug $(n=4)$. Again, a burst release was seen within the first two hours, followed by sustained release for $24 \mathrm{~h}$. The difference in release time of both 
agents can be explained by the solubility of the drugs [30, 31]. The dispersed Fl-Na has a longer elution time since there are three mechanisms that govern the release rate: (i) the rate of dissolution of the Fl-Na crystals/particles; (ii) the rate of diffusion of the Fl-Na molecules through the cross-linked coating matrix; (iii) the porosity of the substrate. It can be argued that for this choice of materials the rate of dissolution of the crystals is the rate determining step, as the drug CAP does not form crystals and is released much faster compared to Fl-Na. Furthermore, the hydrophobic substrate also plays a role. The very hydrophilic Fl-Na is not compatible with the very hydrophobic substrate. However, this incompatibility is decreased by the polyether based coating, which is more polar than the polyolefin Solupor ${ }^{\mathbb{R}}$.

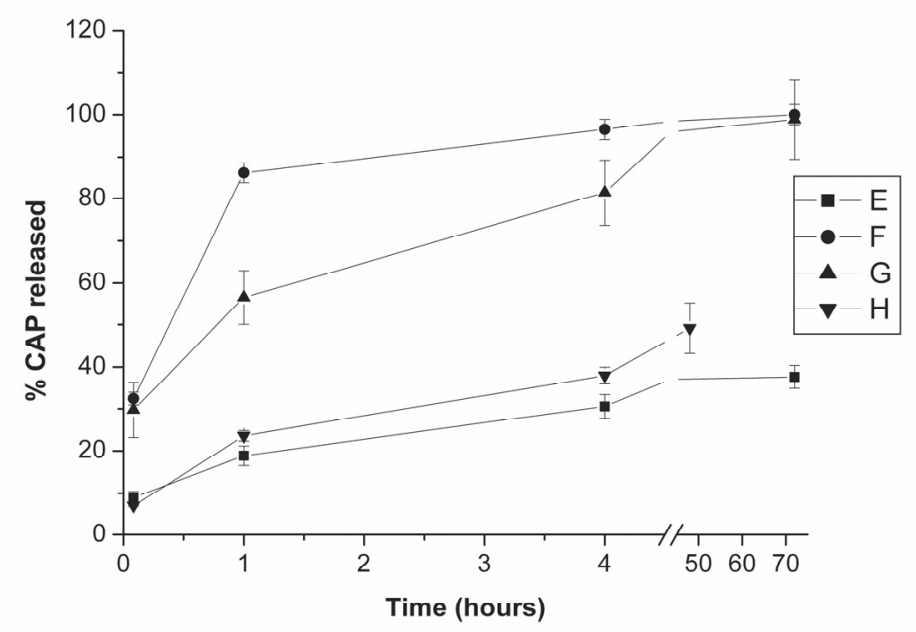

Figure 8.7. The percentage of CAP released from different coated Solupor ${ }^{\circledR}$ substrates $(n=4)$.

Figure 8.7 shows the release of CAP from the coated substrates type E-H $(n=4)$. A distinction is made between the amount of drug that can be released and the amount that is loaded, because not all of the loaded drug is released, as shown in the 70 hours data in Figure 8.7. The figure shows that most of the drug that can be released, is released after 4 hours. Substrate type $\mathrm{F}$ has the largest pores $(0.7 \mu \mathrm{m})$ and has the highest amount of drug released, i.e. $>90 \%$ released within the first hour. In the substrates $\mathrm{E}$ and $\mathrm{H}$, with pore sizes of $0.1 \mu \mathrm{m}$, only released $37 \%$ and $49 \%$ of the entrapped drug respectively. The release profile of substrate $\mathrm{G}$ shows an intermediate, i.e. after 4 hours about $80 \%$ of the drug was released, and after 72 hours the release was finished and almost $100 \%$ was released. 
The effect of multiple drug-loaded coatings on the release profiles of CAP from substrate type $\mathrm{H}$ was examined briefly. This was inspired by the work of K. Anseth, who used multiple coatings with the dye acid orange 8 (AO8) as a model drug to characterize the release of small MW molecules from the designed matrix devices [32] and developed an associate predictive model $[14,33]$. Our preliminary experiments were just designed to determine whether multiple coatings with different concentrations of drug would show differences.
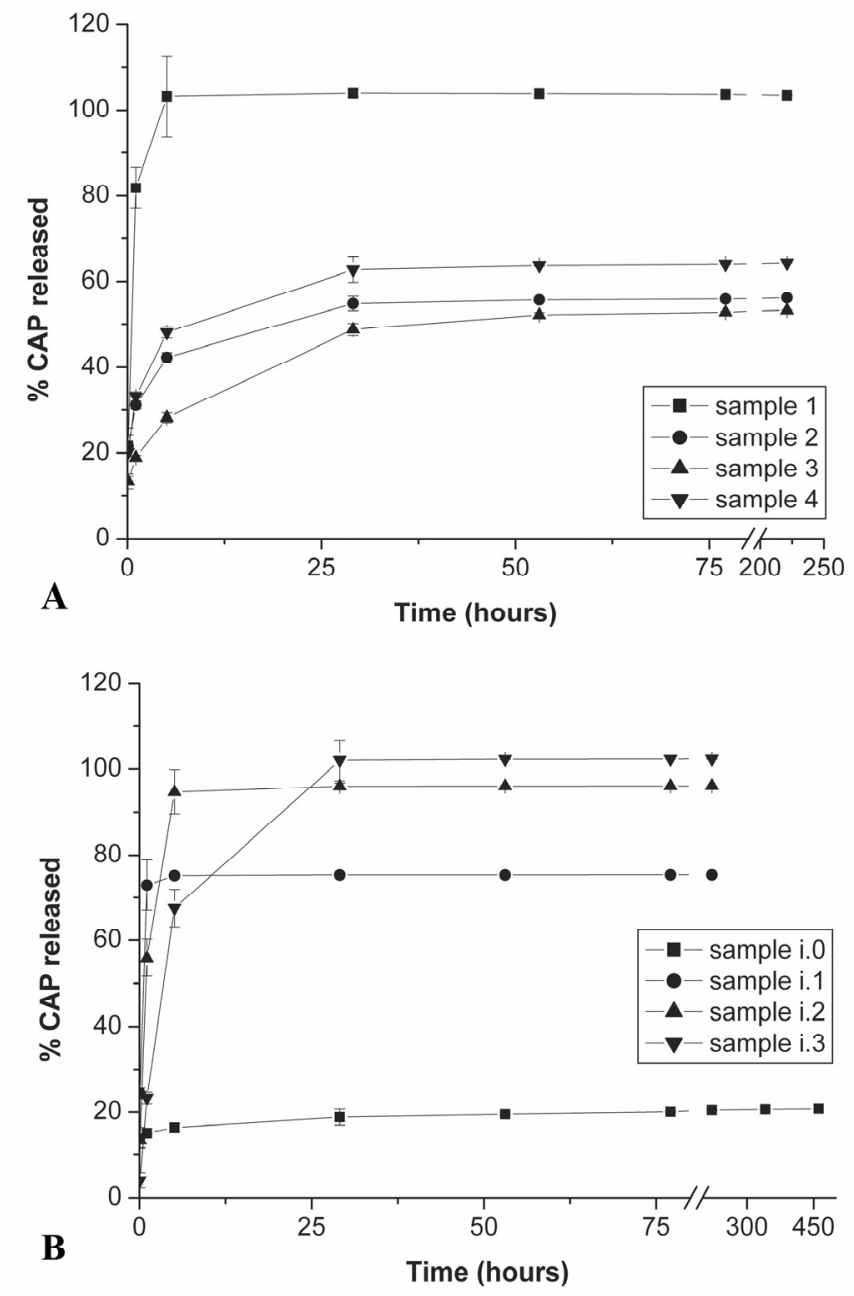

Figure 8.8. (A) The release profile of chloramphenicol from Solupor ${ }^{\circledR}$ substrates with multiple coating layers, (B) the release profile of impregnated Solupor ${ }^{\circledR}$ in combination with (multiple) coating(s). 
The release profiles of multiple drug-loaded coated Solupor ${ }^{\circledR}$ substrates are given in Figure 8.8A. The figure shows that sample 1 (substrate only coated with formulation III) released almost all the entrapped drug within the first 4 hours. Samples $2-4$ have the same first coating, but have an additional topcoat. Sample 2 had a topcoat with $12.7 \mathrm{w} \%$ of CAP, sample 3 had the same topcoat with absence of the drug, and sample 4 had a more cross-linked topcoat without a drug. From the figure it can be seen that applying a topcoat resulted in the fact that not all of the entrapped drug could be released.

The effect of the precipitation of CAP in the pores of the substrate and (multilayer) coatings on the release profiles is given in Figure 8.8B. When the drug was precipitated into the pores of the substrate (sample i.0), the release rate is very slow, i.e. $21 \%$ of the entrapped drug released after 19 days. The profile shows a (small) burst release for the first hour, followed by sustained release. The slow release profile can be explained by the very hydrophobic substrate, and the water will not penetrate into the pores easily. When impregnating the substrate with a formulation after the drug was already precipitated into the pores, the coating provided a route for the water to penetrate the substrate, therefore more of the drug was released compared to sample i.0.

These preliminary experiments would need a much more detailed systematic study in order to determine whether multiple coating layers offers as much value in terms of controlled drug release as the variation in pore size. One can speculate that due to the greater versatility of coatings (differences in polarity, cross-link density and homogeneity of the coating formulation) a much greater flexibility in controlled drug release could be obtained, since with the Solupor ${ }^{\circledR}$ substrate only porosity and film thickness can be varied.

\subsection{Conclusion}

Multiple ways to incorporate drugs into the pores of the Solupor ${ }^{\circledR}$ substrate were developed. Precipitation of the drug into the pores, coating/impregnating the substrate with a drugcontaining formulation, or a combination of both methods is feasible.

In terms of drug release, pore size is a key factor that can be used to control drug release. This is attributed in part to the tortuous path that the drug would have to follow when being released from the fibrous structure of Solupor ${ }^{\mathbb{R}}$. The coating and impregnation matrix is another vehicle to control drug release. It would be particularly useful when the drug is more polar (hydrophilic), since the Solupor ${ }^{\circledR}$ substrate is highly hydrophobic. 
These thin films, coated or uncoated, could be used in a variety of forms in thin films, multilaminated wafers, or tubular rolls. There is much more further work needed in order to better understand the potential of this drug eluting matrix, for example, the effect of biological fluids, the effect of laminated or rolled geometries, as well as this matrix in combination with other materials in implants.

\subsection{Acknowledgements}

The authors would like to thank DSM Solutech for providing the porous substrates, and DSM Resolve for their support with the characterization techniques.

\section{References}

1. Berg M.C., Zhai L., Cohen R.E. and Rubner M.F. Controlled drug release from porous polyelectrolyte multilayers. Biomacromolecules 7 (1) (2006) 357-364.

2. Langer R. New methods of drug delivery. Science 249 (4976) (1990) 1527-1533.

3. Wang C., He C., Tong Z., Liu X., Ren B. and Zeng F. Combination of adsorption by porous CaCO3 microparticles and encapsulation by polyelectrolyte multilayer films for sustained drug delivery. Int $\mathrm{J}$ Pharm 308 (1-2) (2006) 160-167.

4. Hong Y., Gao C., Shi Y. and Shen J. Preparation of porous polylactide microspheres by emulsionsolvent evaporation based on solution induced phase separation. Polym Adv Technol 16 (8) (2005) 622-627.

5. Yang F., Murugan R., Ramakrishna S., Wang X., Ma Y.-X. and Wang S. Fabrication of nanostructured porous PLLA scaffold intended for nerve tissue engineering. Biomaterials 25 (10) (2004) 1891-1900.

6. Kim H.-W., Knowles J.C. and Kim H.-E. Hydroxyapatite porous scaffold engineered with biological polymer hybrid coating for antibiotic Vancomycin release. J Mater Sci 16 (3) (2005) 189-195.

7. Chu L.-Y., Li Y., Zhu J.-H., Wang H.-D. and Liang Y.-J. Control of pore size and permeability of a glucose-responsive gating membrane for insulin delivery. J Control Release 97 (1) (2004) 43-53.

8. McHugh A.J. The role of polymer membrane formation in sustained release drug delivery systems. $\mathrm{J}$ Control Release 109 (1-3) (2005) 211-221.

9. Charnay C., Begu S., Tourne-Peteilh C., Nicole L., Lerner D.A. and Devoisselle J.M. Inclusion of ibuprofen in mesoporous templated silica: drug loading and release property. Eur J Pharm Biopharm 57 (3) (2004) 533-540.

10. Kurtz S.M. The UHMWPE handbook: ultra-high molecular weight polyethylene in total joint replacement, 1st edn. US: Academic Press Inc.; 2004.

11. Fortuin H.M. and Simmelink J.A.P.M. Microporous film of polyethylene and process for the production thereof US5376445 (1994).

12. DSM Solutech, http://www.dsm.com (2007).

13. Fang J.Y., Huang Y.B., Wang H.Y. and Tsai Y.H. Electrically-assisted skin permeation of two synthetic capsaicin derivatives, sodium nonivamide acetate and sodium nonivamide propionate, via rate-controlling polyethylene membranes. Biol Pharm Bull 28 (9) (2005) 1695-1701. 
14. Lu S., Ramirez W.F. and Anseth K.S. Photopolymerized, multilaminated matrix devices with optimized nonuniform initial concentration profiles to control drug release. J Pharm Sci 89 (1) (2000) 45-51.

15. Elisseeff J., Anseth K., Sims D., McIntosh W., Randolph M. and Langer R. Transdermal photopolymerization for minimally invasive implantation. Proc Natl Acad Sci 96 (6) (1999) 31043107.

16. Ward J.H. and Peppas N.A. Preparation of controlled release systems by free-radical UV polymerizations in the presence of a drug. J Control Release 71 (2) (2001) 183-192.

17. Nguyen K.T. and West J.L. Photopolymerizable hydrogels for tissue engineering applications. Biomaterials 23 (22) (2002) 4307-4314.

18. Baroli B. Photopolymerization of biomaterials: issues and potentialities in drug delivery, tissue engineering, and cell encapsulation applications. J Chem Technol Biotechnol 81 (4) (2006) 491-499.

19. Peters R., Litvinov V.M., Steeman P., Dias A.A., Mengerink Y., van Benthem R., de Koster C.G., van der Wal S. and Schoenmakers P. Characterization of UV-cured acrylate networks by means of hydrolysis followed by aqueous size-exclusion combined with reversed-phase chromatography. $\mathrm{J}$ Chromatogr A 1156 (1-2) (2007) 111-123.

20. Studer K., Decker C., Beck E. and Schwalm R. Overcoming oxygen inhibition in UV-curing of acrylate coatings by carbon dioxide inerting: Part II. Progress in Organic Coatings 48 (2003) 101111.

21. Bryant S.J., Nuttelman C.R. and Anseth K.S. Cytocompatibility of UV and visible light photoinitiating systems on cultured NIH/3T3 fibroblasts in vitro. J Biomater Sci Polym Ed 11 (5) (2000) 439-457.

22. Nelson J.D. Simultaneous evaluation of tear turnover and corneal epithelial permeability by fluorophotometry in normal subjects and patients with keratoconjunctivitis sicca (KCS). Trans Am Ophthalmol Soc 93 (1995) 709-753.

23. Lv F.F., Li N., Zheng L.Q. and Tung C.H. Studies on the stability of the chloramphenicol in the microemulsion free of alcohols. Eur J Pharm Biopharm 62 (3) (2006) 288-294.

24. Medicines Evaluation Board, http://www.cbg-meb.nl. Chloramphenicol $0.4 \%$ eye drops, Thea Pharma, Wetteren, Belgium (2007).

25. Dias A.A., Rooijmans M., Litvinov V., Currie E.P.K. and Houben E.J.E. Method for reducing the amount of migrateables of polymer coatings WO06056482 (2006).

26. Williams C.G., Malik A.N., Kim T.K., Manson P.N. and Elisseeff J.H. Variable cytocompatibility of six cell lines with photoinitiators used for polymerizing hydrogels and cell encapsulation. Biomaterials 26 (11) (2005) 1211-1218.

27. Kalakkunnath S., Kalika D.S., Lin H. and Freeman B.D. Viscoelastic characteristics of UV polymerized poly(ethylene glycol) diacrylate networks with varying extents of crosslinking. J Polym Sci Part B: Polym Phys 44 (15) (2006) 2058-2070.

28. Tummers P.H.G., Houben E.J.E., Jansen J.F.G.A. and Wienke D. Evaluation of advanced data analysis techniques on time resolved FT-IR spectroscopy data of photo-copolymerization reactions. Vib Spectrosc 43 (1) (2007) 116-124.

29. Mota M.C., Carvalho P., Ramalho J. and Leite E. Spectrophotometric analysis of sodium fluorescein aqueous solutions. Determination of molar absorption coefficient. Int Ophthalmol 15 (5) (1991) 321326.

30. Narasimhan B. and Peppas N.A. Molecular analysis of drug delivery systems controlled by dissolution of the polymer carrier. J Pharm Sci 86 (3) (1997) 297-304.

31. Huang X. and Brazel C.S. On the importance and mechanisms of burst release in matrix-controlled drug delivery systems. J Control Release 73 (2-3) (2001) 121-136.

32. Lu S. and Anseth K.S. Photopolymerization of multilaminated poly(HEMA) hydrogels for controlled release. J Control Release 57 (3) (1999) 291-300.

33. Lu S., Ramirez W.F. and Anseth K.S. Modeling and optimization of drug release from laminated polymer matrix devices. AIChE 44 (7) (1998) 1689-1696. 


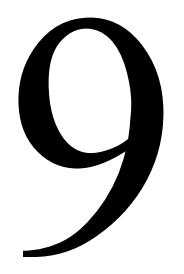

General Discussion 


\section{General Discussion}

Administration of drugs to both anterior and posterior tissues of the eye is troublesome. Topical drug delivery is commonly carried out via eye drops, but this method has many disadvantages. This thesis describes the research and development of a novel ocular drug delivery device. The device consists of a hydrogel coated coil, which can be loaded with a drug. It is placed in the lower conjunctival fornix, where it releases its drug gradually to the tear film, and from there to the anterior (and possibly also posterior) ocular tissues. First, the preparation of the device was described in detail, as well as the incorporation of drugs into the coating layer. Then, several methods were provided, not only to increase the capacity, but also to extend the release time and to obtain more control over the release profile. This was achieved by filling the interior of the coil with a drug-loaded material.

Several methods to achieve filling of the luminal space of the OphthaCoil with an additional amount of drug were explored. Specifically, short wires with a drug-loaded hydrogel coating, drug-loaded hydrogel microspheres, drug-loaded hydrogel rods, and drug-loaded textile fibers were examined. Wires with a drug-loaded hydrogel coating are far from the ideal method, because only the coating of the wire can contain a drug, which is only a fraction of the total lumen. The wire itself fills already most of the lumen (Chapter 2 and 3). Also, the flexibility of the device is decreased significantly after introducing these wires in the lumen, and this can adversely influence the tolerance of the device in the fornix. Using drug-loaded microspheres in the interior of the coil, the drug capacity of the device can be increased significantly, and can be dosed simply by varying the number of microspheres in the interior. On the other hand, this method is complex, time consuming and hence expensive (Chapter 5). The use of a drugloaded polymer rod is less labour-intensive and complex, but special care must be taken during the polymerization, since the diameter of the rod is very small. Moreover, there is a significant danger that toxic unreacted monomers leach away from the device's core towards the tear film (Chapter 6 and 7). Use of a textile fiber, impregnated with the drug of choice, within the device's lumen probably provides the most suitable and safe solution to the capacity problem. We noticed, however that it is difficult to achieve truly reproducible drug release profiles. This may indicate that further improvements in our methods to impregnate the drug in the fiber and/or to fill the device, are required (Chapter 7). The drug release from a coil filled by the latter two methods are comparable (demonstrated in Chapter 7).

An advantage of the device compared to the commonly used eye drops is the higher bioavailability of the drug. The exact extent of drug absorption in the ocular tissues has not 
been investigated yet, but the in vivo experiment with the mydriatic agents showed that using approximately $200 \mu \mathrm{g}$ of mydriatics (phenylephrine and tropicamide) can give mydriasis in Beagle dogs. To obtain mydriasis in the human eye, normally three drops of phenylephrine hydrochloride $(2.5 \% \mathrm{w} / \mathrm{v})$, and three drops of tropicamide $(0.5 \% \mathrm{w} / \mathrm{v})$ are administered, which accounts for a total drug amount of $4500 \mu \mathrm{g}$, assuming that the volume of an eye drop is $50 \mu \mathrm{L}$. This is an indication that the device can obtain a much better drug absorption into the ocular tissues compared to eye drops. As a result of the higher bioavailability and the correct dosing of the drug, less frequent administrations are necessary, and this can increase the patient compliance. However, in literature, it was mentioned that insertion and removal of a device once a week can be quite difficult for users (Y. Ali and K. Lehmussaari, Adv Drug Deliv Rev 58 (2006) 1258-68). It is possible that the removal of the device and possible insertion of a new one, can be combined with control examinations in the clinic. But this depends on the drug release period; for example, once a month or even less in the treatment of chronic diseases.

An advantage of the device over other ocular inserts is the shape, i.e. the length and the flexibility, which ensure that the device can not move within the lower conjunctival fornix. In addition, different drugs can be incorporated into the device simultaneously, and the integrity of the device in both the wet and dry state is ensured by the metal wire.

At first sight, the use of an ocular device that consists of a metallic core may raise questions although the wire is very thin $(76 \mu \mathrm{m})$ and the coil is extremely flexible. In the OphthaCoil, there is however, no contact between the metal and the ocular epithelium, because of the hydrogel coating and the polymer caps. The essential role of the coiled metallic wire is that it guarantees the device's integrity throughout the application time window; there is no degradation of the device itself, contrary to what has been encountered with all-polymeric hydrogel ocular inserts. Furthermore, we are aware of other ocular inserts with a metallic core, such as the I-vation ${ }^{\mathrm{TM}}$ TA coated screw that was invented by Surmodics (Eden Prairie, MN, USA), as a vehicle for drug release to the vitreous body. Other possible positive aspects of the use of a metallic substrate in the OphthaCoil are the possible removal of the device with a magnet (instead of tweezers), and the metallic coil provides X-ray visibility, which is an extra safety issue. We do recognize that the clinical safety of the OphthaCoil, especially for longterm applications, still remains to be established. Additional in vivo experiments must be performed to assure the safety of the device in the eye. On the other hand, for short-term drugrelease up to several days or a week, we do not see any objections in using the metal wire, as the Beagle dogs did not show any signs of possible irritation or avoidance to the wear of the device. The porous material, described in Chapter 8 , could be very useful for the long term 
applications, as this material is biocompatible and well-known as an implant material in the body.

Another disadvantage of the coil is the fact that removal of the device is necessary after the drug is released. The insertion and removal procedure probably must be done by healthcare workers, or after a training protocol of the patient. One aspect is that the insertion and removal of the insert can possibly be combined with control examinations in the hospital or eye centre, as mentioned before. Additional analysis are required as well to investigate the device's price for a whole treatment compared to the treatment via eye drops.

In veterinary medicine, for example in dogs, cats or horses, the third eyelid can cause some problems. It moves over the surface of the eye when the eyeball is retracted. Some functions of this eyelid are the protection of the cornea and removal of foreign material from the corneal surface. A possible solution for the loss of the device in the lower conjunctival fornix is the use of a soluble suture (e.g. Vicryl), also discussed in Chapter 6. The major disadvantage of the use of a suture, is the fact that the animal must undergo general anesthesia. On the contrary, this method can be considered for long-term drug release, for example to treat uveitis in the equine eye. In humans we do not foresee this problem, as humans do not have third eyelids, but the presence of the device overnight in the human eye must still be verified in clinical experiments.

There are several applications conceivable for this ocular device, both in human and veterinary medicine. For example, in the treatment or prevention of ocular infections/inflammation, drugs must mostly be delivered up to 14 days. In this thesis, it was demonstrated that the device filled with a drug-loaded hydrogel rod, could deliver drugs to the tear fluid for at least 72 hours in Beagle dogs. This may lead to a frequency of administration of only twice a week, instead of several times a day, and this could increase the patient compliance.

Another potential application for this device is in pre-operative drug delivery. An example is found in cataract surgery, where three types of drugs must be administered several times within 1-2 hours prior to the surgery. Here, one single coil, for example filled with a drug-loaded fiber, could provide a release of these different drugs within this timeframe.

For posterior drug delivery, the coil could be placed along the sclera (i.e. in a scleral tunnel), where it releases its drug which will be diffused into the vitreous. Possibly, the coil can be reloaded with drugs or a drug-containing polymer using an injection. However, for long-term drug delivery (as in more than several days), further research must be done to increase the drug capacity and to extend the release time, as well as to investigate the presence and tolerance of the device in the eye. 
We believe that we have designed a novel and promising device for the sustained delivery of drugs to the eye. The device was tested in vitro and in vivo. At this point, a clinical application for the device must be chosen and further clinical trials towards this application must be performed. For example, additional clinical tests to investigate the tolerance of the device in the eye, and drug release from the device into the different ocular tissues, like the drug concentration in the anterior and vitreous chamber in time. Hopefully at one day, this research will lead to a commercial product, which is used in the clinic.

Finally, the use of a highly porous substrate as a matrix for the delivery of drugs was investigated as well. This substrate consists of UHMWPE, which is one of the most common used polymer implanted in the body. It can be used in various appearances, e.g. flat or rolled in a tubular device, and for multiple applications, e.g. intravascular drug delivery, mucosal drug delivery, or delivery of drugs to the ear or eye. In the eye the material can be used flat, in the lower conjunctival fornix, or rolled and placed subscleral, for the posterior delivery of drugs. Also, a combination of both approaches described in this thesis would be an option. The OphthaCoil could be filled with a drug-loaded rolled piece of Solupor ${ }^{\mathbb{R}}$. This drug-loaded porous material is still in the development phase. Initial in vitro experiments have shown promise in that this porous material provides a number of different modalities to control drug release, e.g. pore size, impregnation of a drug-loaded formulation and its cross-link density, and precipitation and crystallization of drugs in the pores of the substrate. In vivo experiments must demonstrate the presence and tolerance of the material in the eye as well as the drug concentration in the different ocular tissues. 
$142 \mid$ Chapter 9 


\section{Appendix I. Title page patent}

(12) INTERNATIONAL APPLICATION PUBLISHED UNDER THE PATENT COOPERATION TREATY (PCT)

(19) World Intellectual Property Organization International Bureau

(43) International Publication Date 18 January 2007 (18.01.2007)

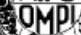

PCT

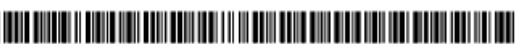

(10) International Publication Number WO 2007/006427 A1
(51) International Patent Classification: A61L 31/10 (2006.01) A61K 9/50 (2006.01) A6IF 2/14 (2006.01)

(21) International Application Number: PCT/EP2006/006355

(22) International Filing Date: 30 June $2006(30.06 .2006)$

(25) Filing Language:

English

(26) Publication Language:

English

(30) Priority Data:

05076580.9

05077210.2

11 July 2005 (11.07.2005) 27 September 2005 (27.09.2005)

EP

EP

11) Applicant (for all designated States except US): DSM IP ASSETS B.V. [NL/NL]; Het Overloon 1, NL-6411 Te Heerlen (NL).

(72) Inventors; and

75) Inventors/Applicants (for US only): DIAS, Aylvin, Jorge, Angelo, Athanasius [NL/NL]; Pletzersstraaat 17D, NL-6213 HE Maastricht (NL). KOOLE, Levinus, Hendrik [NL/NL]; Bolderik 9, NL6271 EC Gulpen (NL). PIJLS, Rachel, Theodora [NL/NL]; Markt 70, NL-6161 GL Geleen (NL).
(74) Agent: HABETS, W.J.A.; DSM Intellectual Property, P.O. Box 9, 6160 MA Geleen (NL).

(81) Designated States (unless otherwise indicated, for every kind of national protection available): $\mathrm{AE}, \mathrm{AG}, \mathrm{AL}, \mathrm{AM}$, $\mathrm{AT}, \mathrm{AU}, \mathrm{AZ}, \mathrm{BA}, \mathrm{BB}, \mathrm{BG}, \mathrm{BR}, \mathrm{BW}, \mathrm{BY}, \mathrm{BZ}, \mathrm{CA}, \mathrm{CH}, \mathrm{CN}$, $\mathrm{CO}, \mathrm{CR}, \mathrm{CU}, \mathrm{CZ}, \mathrm{DE}, \mathrm{DK}, \mathrm{DM}, \mathrm{DZ}, \mathrm{EC}, \mathrm{EE}, \mathrm{EG}, \mathrm{ES}, \mathrm{FI}$, GB, GD, GE, GH, GM, HN, HR, HU, ID, IL, IN, IS, JP, KE, KG, KM, KN, KP, KR, KZ, LA, LC, LK, LR, LS, LT, LU, LV, LY, MA, MD, MG, MK, MN, MW, MX, MZ, NA, NG, NI, NO, NZ, OM, PG, PH, PL, PT, RO, RS, RU, SC, SD, SE, SG, SK, SL, SM, SY, TJ, TM, TN, TR, TT, TZ, UA, UG, US, UZ, VC, VN, ZA, ZM, ZW.

(84) Designated States (unless otherwise indicated, for every kind of regional protection available): ARIPO (BW, GH, GM, KE, LS, MW, MZ, NA, SD, SL, SZ, TZ, UG, ZM, $\mathrm{ZW}$ ), Eurasian (AM, AZ, BY, KG, KZ, MD, RU, TJ, TM), European (AT, BE, BG, CH, CY, CZ, DE, DK, EE, ES, FI, FR, GB, GR, HU, IE, IS, IT, LT, LU, LV, MC, NL, PL, PT, RO, SE, SI, SK, TR), OAPI (BF, BJ, CF, CG, CI, CM, GA, GN, GQ, GW, ML, MR, NE, SN, TD, TG).

Published:

- with international search report

For two-letter codes and other abbreviations, refer to the "Guidance Notes on Codes and Abbreviations" appearing at the beginning of each regular issue of the PCT Gazette. particular for controlled release of drugs to the eye. Delivery of drugs to the anterior side of the eye is routinely done with eye drops, but this method results in low bioavailability and low patient compliance. Devices that address these problems have been described for the delivery of drugs to the eye. One of such devices, called the OphthaCoil, consists of a thin metallic wire, which is coiled and carries a drug-loaded adherent hydrogel coating on its surface. Surprisingly it has now been found that the drug loading capacity can be dramatically increased by filling the lumen of the coil with micro-particles such as microspheres that contain the drug of choice. 
Summary

Samenvatting

List of Publications

Curriculum Vitae

Dankwoord 


\section{Summary}

Topical ocular drug delivery is routinely done via eye drops. This method is well-accepted and for most patients easy to use. However, this method has several drawbacks. It results in low bioavailability, because the rate and extend of absorption of the drug is severely limited due to spilling and clearance of the drop. Thus, frequent administrations of high drug concentrations are necessary, which may cause toxic side effects and low patient compliance.

The use of an ocular device for the delivery of drugs would be a solution to the problems of eye drops. An ocular device, for example placed in the lower conjunctival fornix, can release its drug gradually over a period of time in situ. This would result in a higher bioavailability, as the drug is exposed in a much more sustained manner to the ocular tissues. Better patient compliance may be achieved, especially when installation of an ocular insert provides sustained drug release during, for example, several days. Using an ocular insert is also a solution for patients who have difficulties with the self-administration of the drugs, like the elderly, children and infirm. Also in the veterinary medicine, an ocular drug delivery device has potential, for example to treat common eye infections in dogs, cats or horses.

This thesis describes the development of a novel ocular drug delivery device, called the OphthaCoil. The device is essentially a coiled wire with the following characteristics: i) the device is based on hydrogel technology; in this case, the hydrogel is applied as a coating on a thin metallic wire; ii) the hydrogel coating contains a drug (e.g. an antibiotic); after contact with tear fluid, the coating will swell and releases its drug; iii) the ends of the coil are closed with polymer caps; iv) the lumen of the coil provides space for an additional drug reservoir, what increases the capacity of the device. The dimensions of the device are $16 \mathrm{~mm}$ in length and appr. $0.6 \mathrm{~mm}$ in diameter. The device is non-biodegradable to prevent possible disintegration of the device or leakage of the drug after swelling of the hydrogel. The preparation of the device is described in detail in Chapter 2. The hydrogel Slipskin ${ }^{\circledR}$ was used for the coating. Slipskin ${ }^{\circledR}$ consists of a hydrophilic and a hydrophobic monomer. The ratio of these monomers can be varied to obtain coatings with different hydrophilicities. The release of drugs from these coatings is affected by the time and degree of swelling of the hydrogel coating. This was shown for the dye fluorescein. All different coatings showed initial fast release in the first hour, but the most hydrophilic coating released the most amount of drug. Preliminary in vivo pilot experiments with the OphthaCoil were shown in Chapter 2 as well. A coil with fluorescein in the coating was placed in the lower conjunctival fornix of a volunteer, and it could be seen that the fluorescein was distributed via the tear film all over the eye within a few minutes. Also, coils with the mydriatic agent atropine in the coating, were placed in the 
eye of two volunteers and the diameter of the pupil was followed over time. There was no pupil-widening effect seen in both volunteers, but when two coils were placed simultaneously in the eye of one volunteer, the mydriatic effect was seen within several minutes. The effect was found to persist for over two hours.

Chapter 2 also describes the first method of using the interior of the coil as an additional reservoir for the drug. One, two or three thin, straight pieces of hydrogel coated wires, loaded with the dye fluorescein, were placed in the interior of a coil, loaded with a fluoresceincontaining coating. The release profile showed again a burst release for the first hour, but afterwards sustained release was seen for more than 4 days. Then, the preparation of two coils with different amounts of the antibiotic chloramphenicol in the hydrogel coating was described. The interior of the coils were filled identically with three drug-loaded coated wires. A zone of inhibition assay demonstrated that chloramphenicol was released from both coils and that the chloramphenicol was still active after the coating procedure.

Finally, a technique was found to round off sharp ends of the coil by means of a UVpolymerizable cyanoacrylate. Using these sealings, the potential damage of the coil to the ocular surface can be minimized. Another advantage is the fact that the swelling medium has to diffuse through the windings and that the drug release from the interior of the coil via the ends of the coil can be avoided.

In Chapter 3, the hydrogel coating of the coil and the wires in the interior were loaded with the anti-infective pradofloxacin. The initial release in vitro was fast. However, after 48 hours of immersion, the concentration was still far above the MIC-value. The tolerance of the device in the canine eye was evaluated as well. Three Beagle dogs received a 'blank' coil, as with no active agents, under their lower eyelid. The animals remained calm and did not show any signs of discomfort due to the coils. After 24 hours, all three animals lost their device. Our hypothesis that during sleep the movement of the third eyelid over the eye is the reason for the loss of the coils overnight was supported by an animal that had its third eyelids removed. Therefore, an experiment was done where coils loaded with pradofloxacin were inserted for 8 hours. Then, the coils were removed and stored until the next day, where the same coils were inserted in the same eyes for another 8 hours. Tear fluid samples with Schirmer strips and HPLC characterization showed that after 16 hours, the concentration of pradofloxacin in the tear fluid was still above the MIC-value of the anti-infective.

A clinical evaluation of the device in the human eye was described in Chapter 4. The response

to installation, 2h-wear, and removal of the device were evaluated. Ophthalmologic examinations, as well as photographs of the capillary veins, showed a normal appearance of the ocular tissues in all volunteers. Questionnaires filled by the volunteers revealed that most of the 
volunteers did feel the presence of the device in their eye, but in most cases the feeling was characterized as 'a little'. We believe that these responses were reminiscent of responses seen routinely with new wearers of contact lenses.

In Chapter 5 drug-loaded microspheres were introduced in the interior of the coil. Microspheres made from the monomer HEMA solely, or in combination with NVP, were prepared by suspension polymerization. The microspheres were soaked in a highly concentrated solution with a drug, after which they were lyophilized. The release was studied in vitro in a pump set-up, where Simulated Tear Fluid was perfused along the devices. Filling the interior with fluorescein sodium-loaded microspheres resulted in a capacity increase of $500 \%$. Also, the release time was extended from 90 minutes (from a coil with an empty lumen) to 5 hours at least. This effect was much less with the drug ciprofloxacin, which could not be dissolved very well in a medium which obtains swelling of the microspheres. Also, three-point bending tests were done with several coils to investigate the structural stiffness of the device. It was seen that the flexibility of coils with microspheres in the interior was similar to the coils with an empty interior. Coils with three coated wires inside showed clearly a decrease in flexibility.

A third approach of filling the interior of the coil to increase the capacity and release time was described in Chapter 6. Here, a pradofloxacin-loaded polymer rod prepared from the monomer HEMA was inserted in the coil. The in vitro release in the perfusion system was compared to the in vivo release to the tear film of the eyes of Beagle dogs. To ensure the presence of the coil during the experiment, the coils were fixated in the eyes using one stitch. The release curves of pradofloxacin were similar. Drug levels declined steadily, but in vivo measurements showed a longer release time compared to the in vitro data, i.e. 72 hours compared to 32 hours. This may be attributed to the relative slow refresh rate of the tear fluid in the eye compared to the in vitro flow. Chapter 7 compares the release of chloramphenicol and cyclosporine A from coils filled with a polymer rod, with the release from coils filled with drug-loaded textile fibers. In the latter method, the fibers were soaked into a highly concentrated drug solution, after which they were dried and introduced into the interior of the coil. In vitro data of chloramphenicol shows that the capacity of the device could be increased with $300 \%$ using the polymer rod, and $500 \%$ using the textile fiber, both compared to a coil with an empty lumen. We noticed, however that it is difficult to achieve truly reproducible drug release profiles with coils filled with the textile fibers. This may indicate that further improvements to impregnate the drug in the fiber and/or to fill the device, are required.

Then, coils containing cotton fibers, soaked into a solution of phenylephrine and tropicamide, were placed in the eyes of eight Beagle dogs. Within one hour, a clear effect was seen on the 
dilation of the pupil and its response to light. This effect persisted for over 4 hours, even when the coil was lost during the experiment.

In addition, coils with a larger diameter and length $(1.2 \mathrm{~mm}$ and $30 \mathrm{~mm}$ respectively) were prepared for the insertion in the equine eye. In vitro release of the immuno-suppressor cyclosporine A from these coils showed that the capacity could be increased with a factor 10 using the polymer rod, and a factor 18 using the textile fibers, again both compared to an empty coil. The release of cyclosporine A decreased gradually over time for at least 72 hours. Furthermore, two horses received a coil under their lower eyelid. The insertion was uneventful and easy, without the use of anaesthetics. After 5 hours, the coils were still in place, but overnight the coils were lost again.

In Chapter 8 a switch has been made towards another concept for the controlled delivery of drugs. The use of a porous UHMWPE film was investigated. The film, named Solupor ${ }^{\circledR}$, was loaded with chloramphenicol via different methods. It was possible to completely fill the pores of the substrate by different loading techniques. Initial in vitro experiments have shown promise in that this porous material provides a number of different modalities to control drug release, e.g. pore size, impregnation of a drug-loaded formulation and its cross-link density, and precipitation and crystallization of drugs in the pores of the substrate. This material is not limited for the use in ocular drug delivery, but can also been utilized in other applications, like intravascular or mucosal drug delivery, or drug delivery to the ear. This material has much potential for the controlled delivery of drugs, because of its high porosity (up to $85 \%$ ). However, the use of this material as a platform for drug release is still in development, and future in vivo experiments must demonstrate the presence and safety of the device, as well as the drug concentrations in the different ocular tissues. 


\section{Samenvatting}

Het toedienen van medicijnen aan de voorkant van het oog geschiedt normaliter door middel van oogdruppels. Deze methode is alom geaccepteerd en voor de meeste patiënten makkelijk in het gebruik. Deze methode heeft echter verschillende nadelen. Het resulteert in een lage hoeveelheid van het medicijn dat wordt geabsorbeerd door de weefsels, omdat de absorptie wordt beperkt door verspilling en klaring van de oogdruppel. Vandaar dat regelmatige toedieningen met zeer hoge medicijn concentraties nodig zijn, hetgeen gevaarlijke bijwerkingen kan veroorzaken en vaak resulteert in beperkte therapietrouw.

Het gebruik van een matrix voor de toediening van deze medicijnen zou een oplossing kunnen zijn voor de problemen die voorkomen bij oogdruppels. Een matrix, bijvoorbeeld geplaatst achter het onderste ooglid, kan zijn medicijn(en) geleidelijk afgeven in het oog zelf. Het gevolg is een betere absorptie van het medicijn in de weefsels van het oog. Een betere therapietrouw kan worden bereikt, wanneer een matrix een medicijn voor bijvoorbeeld meerdere dagen kan afgeven. Het gebruik van een matrix is ook een oplossing voor patiënten die moeilijkheden hebben met de toediening van oogdruppels, zoals ouderen, kinderen en gehandicapten. Eveneens in de diergeneeskunde heeft een matrix vele mogelijkheden, bijvoorbeeld bij de behandeling van ooginfecties in honden, katten of paarden.

Dit proefschrift beschrijft de ontwikkeling van een nieuw oogimplantaat, genaamd de "OphthaCoil". Het bestaat uit een spiraal met de volgende eigenschappen: i) de werking berust op hydrogel technologie; in dit geval wordt de hydrogel toegepast als een coating op een dunne metalen draad; ii) de hydrogel coating bevat een medicament (bijvoorbeeld een antibioticum); na contact van de spiraal met het traanvocht, gaat de coating zwellen en wordt het medicament afgegeven; iii) de uiteinden van de spiraal zijn gedicht met polymere dopjes; iv) het lumen van de spiraal verschaft ruimte voor een aanvullend reservoir voor medicijnen, wat de capaciteit van het implantaat vergroot. De dimensies van de spiraal zijn $16 \mathrm{~mm}$ in de lengte bij ongeveer $0,6 \mathrm{~mm}$ in diameter. Het implantaat is niet degradeerbaar, zodat mogelijke desintegratie van de spiraal of lekkage van het medicijn nadat de hydrogel gezwollen is, wordt voorkomen. De vervaardiging van het implantaat is tot in detail beschreven in Hoofdstuk 2. De hydrogel Slipskin ${ }^{\circledR}$ is gebruikt als coating op de dunne metalen draad. Slipskin ${ }^{\circledR}$ bestaat uit een hydrofiel en een hydrofoob monomeer. De verhouding tussen deze monomeren werd gevarieerd, zodat coatings met verschillende hydrofiliciteiten konden worden verkregen. De afgifte van medicijnen van de verschillende coatings werd beïnvloed door de snelheid en mate van zwelling van deze lagen. Dit werd gedemonstreerd voor de kleurstof fluoresceïne. Alle 
verschillende coatings lieten in het eerste uur een snelle eerste afgifte van de kleurstof zien, maar de meest hydrofiele coating had het meeste medicijn afgegeven.

Tevens worden in Hoofdstuk 2 enkele in vivo experimenten met de OphthaCoil beschreven. Een spiraal met een coating geladen met fluoresceïne, werd geplaatst achter het onderste ooglid van een vrijwilliger. De fluoresceïne werd duidelijk binnen enkele minuten via het traanvocht over het oog verdeeld. Ook spiralen met het mydriaticum atropine in de coating werden geplaatst in het rechter oog van twee vrijwilligers. De diameter van de pupillen werd gevolgd in de tijd, maar er was geen dilatatie van de pupil te zien bij beide vrijwilligers. Wanneer twee spiralen tegelijkertijd bij een vrijwilliger achter het ooglid werden geplaatst, was binnen enkele minuten dilatatie van de pupil waar te nemen. Dit effect hield meer dan twee uur aan.

Hoofdstuk 2 beschrijft bovendien een eerste methode om de kern van de OphthaCoil te gebruiken als een additioneel reservoir voor medicijnen. Een, twee of drie dunne, rechte stukken van een gecoate draad, geladen met fluoresceïne, werden in een spiraal geplaatst, waarvan de draad dezelfde, met fluoresceïne geladen, coating had. Het afgifte profiel liet in het eerste uur een snelle, hoge afgifte zien, gevolgd door langdurige afgifte gedurende meer dan 4 dagen. Vervolgens wordt de vervaardiging van twee spiralen met een verschillende hoeveelheid van het antibioticum chloramfenicol in de coating beschreven. De kern van beide spiralen werden gevuld met drie identieke draden, tevens geladen met chloramfenicol. Een analyse van de remmingszone van bacteriën heeft aangetoond dat chloramfenicol was afgegeven van beide spiralen, en dat dit antibioticum nog steeds werkzaam was na de vervaardiging van de OphthaCoil.

Tenslotte is een techniek gevonden om de scherpe uiteinden van de spiraal af te ronden door gebruik te maken van een UV uithardende cyano-acrylaat. Met het gebruik van deze afdichtingen wordt het potentiële gevaar dat de spiraal de weefsels van het oog zal beschadigen geminimaliseerd. Een ander voordeel van deze afdichtingen is dat het traanvocht eerst door de windingen van de spiraal naar binnen moet diffunderen, zodat het medicijn in het lumen van de spiraal niet via de uiteinden kan worden afgegeven.

In Hoofdstuk 3 wordt het anti-infectie middel pradofloxacin aangebracht in de coating van de spiraal en de draden binnenin. De in vitro afgifte was snel, echter na 48 uur was de concentratie nog steeds ruim boven de MIC-waarde. Tevens is de werking van de OphthaCoil in het oog van een hond geëvalueerd. Drie Beagle honden kregen een OphthaCoil zonder geneesmiddel achter het onderste ooglid geplaatst. De dieren bleven kalm en vertoonden geen symptomen van ongemak als gevolg van de implantaten. Na 24 uur bleek dat alle dieren de spiraal verloren waren. Onze hypothese hiervoor is dat tijdens de slaap het derde ooglid over het oog beweegt en zo de spiraal uit het oog heeft geschoven. Dit werd bekrachtigd door een hond die beide 
derde oogleden verwijderd had en waar de spiraal 24 uur na inbreng nog steeds aanwezig was. Vervolgens is een experiment uitgevoerd waarbij spiralen geladen met pradofloxacin gedurende 8 uur in het oog van honden waren geplaatst. Daarna werden de spiralen verwijderd en opgeslagen tot de volgende dag, waarbij dezelfde spiraal nogmaals voor 8 uur in hetzelfde oog werd geplaatst. Traanvocht samples werden afgenomen met Schirmer strips en HPLC metingen laten zien dat na 16 uur de concentratie van pradofloxacin in het traanvocht nog steeds boven de MIC-waarde van het medicijn ligt.

Een klinische analyse van de OphthaCoil in het menselijk oog is beschreven in Hoofdstuk 4. Reacties op het plaatsen, 2 uur dragen en verwijderen van het implantaat werden geëvalueerd. Oogheelkundige onderzoeken, alsmede foto's van de haarvaten in het oog, duidden bij alle vrijwilligers niet op veranderingen van verschillende weefsels in het oog. Vragenlijsten ingevuld door deze vrijwilligers lieten zien dat de meeste vrijwilligers de OphthaCoil in het oog voelden zitten, maar in de meeste gevallen werd het gevoel aangeduid als "een beetje". Wij geloven dat deze reacties vergelijkbaar zijn met reacties die optreden bij nieuwe dragers van contact lenzen.

In Hoofdstuk 5 worden microbolletjes, geladen met een medicijn, geïntroduceerd in de kern van de spiraal. De microbolletjes werden gemaakt door middel van een suspensie polymerisatie van ofwel het monomeer HEMA, ofwel een combinatie van HEMA en NVP. De microbolletjes werden gedrenkt in een zeer geconcentreerde medicijn oplossing, waarna ze werden gevriesdroogd. De afgifte van het medicijn werd bestudeerd in een opstelling waarbij kunstmatig traanvocht langs de spiraal werd gepompt. Door de kern van de spiraal te vullen met microbolletjes geladen met natrium-fluoresceïne, werd de capaciteit van het implantaat vergroot met $500 \%$. Ook de duur van de afgifte was verlengd van 90 minuten, bij een spiraal met een leeg lumen, naar minstens 5 uur. Dit effect was niet te zien bij het medicijn ciprofloxacine, dat niet voldoende kon worden opgelost in een medium waar de microbolletjes in zwellen. Tevens worden in dit hoofdstuk buigtesten met verschillende spiralen beschreven om de starheid van het implantaat te onderzoeken. Er werd waargenomen dat de flexibiliteit van spiralen met microsferen in de kern vergelijkbaar was met spiralen met een lege kern. Spiralen met gecoate draden in de kern vertoonden een duidelijke afname in flexibiliteit.

Een derde methode voor het vullen van de kern van de spiraal om de capaciteit en de duur van de afgifte te vergroten is beschreven in Hoofdstuk 6. Een polymeer staafje geladen met pradofloxacin werd gemaakt uit het monomeer HEMA en werd in de kern van de spiraal geplaatst. De in vitro afgifte van het medicijn is vergeleken met de in vivo afgifte in het traanvocht van de honden. Om de aanwezigheid van de spiraal in het oog van de honden te garanderen tijdens het experiment werden de spiralen met een enkele hechting aan het oog 
vastgemaakt. De afgifte profielen van beide experimenten waren vergelijkbaar. Medicijn concentraties namen gestaag af, maar de in vivo metingen vertoonden een langere afgifte tijd dan de in vitro metingen, namelijk 72 uur in vivo in vergelijking met 32 uur in vitro. Dit kan mogelijk worden toegeschreven aan de relatief langzame verversing van het traanvocht in het oog, vergeleken met de in vitro verversing van het kunstmatig traanvocht.

Hoofdstuk 7 vergelijkt de afgifte van de medicijnen chloramfenicol en cyclosporine A uit spiralen gevuld met een polymeer staafje met de afgifte van spiralen gevuld met textielvezels. Voor de laatste methode worden de vezels gedrenkt in een geconcentreerde oplossing van het medicijn, waarna ze worden gedroogd en in de kern van de spiraal worden geplaatst. In vitro afgifte van chloramfenicol laat zien dat de capaciteit van het implantaat met 300\% vergroot kan worden door het gebruik van het polymeren staafje, en 500\% door het gebruik van textielvezels, beide vergeleken met een lege spiraal. We hebben echter opgemerkt, dat het moeilijk is om reproduceerbare metingen te verrichten met spiralen gevuld met de textielvezels. Dit duidt erop dat verdere verbeteringen nodig zijn om het medicijn in de vezels te impregneren en/of in de methode de spiraal te vullen met de vezels.

Tevens zijn spiralen, gevuld met textiel vezels gedrenkt in een oplossing met fenylefrine en tropicamide, geplaatst in het rechter oog van 8 Beagle honden. Binnen 1 uur was bij alle honden een duidelijk effect te zien van de verwijding van de pupil en zijn reactie op licht. Dit effect hield minstens 4 uur aan, ook wanneer de spiraal tijdens het experiment verloren was geraakt.

Tenslotte zijn spiralen geprepareerd met een grotere diameter en lengte $(1,2 \mathrm{~mm}$ en $30 \mathrm{~mm}$ respectievelijk) voor de plaatsing in het oog van een paard. In vitro afgifte van de immunosuppressor cyclosporine A laat zien dat de capaciteit van het implantaat, vergeleken met een spiraal met een lege kern, met een factor 10 vergroot kan worden door gebruik te maken van het geladen polymeer staafje, en met een factor 18 gebruikmakend van de textielvezels. De hoeveelheid cylcosporine A afgegeven van de spriaal daalt geleidelijk in de tijd voor tenminste 72 uur. Tevens ontvingen twee paarden een spiraal achter het onderste ooglid. Het inbrengen verliep rustig en eenvoudig, zonder gebruik van verdovingsmiddelen. Na 5 uur waren de implantaten nog steeds aanwezig onder het ooglid, maar na de nacht waren de spiralen, net als bij de Beagle honden, verloren.

In Hoofdstuk 8 is een omschakeling gemaakt naar een ander concept voor gecontroleerde afgifte van medicijnen. Het gebruik van een zeer poreus materiaal, genaamd Solupor ${ }^{\circledR}$, werd onderzocht. Het materiaal was op verschillende manieren geladen met chloramfenicol. Het was mogelijk om de poriën volledig te vullen door middel van verschillende technieken. De in vitro experimenten laten zien dat er mogelijk meerdere manieren zijn om de afgifte van medicijnen 
te controleren, bijvoorbeeld de poriegrootte, impregnatie van een formulering geladen met een medicijn, en de crosslink dichtheid hiervan, alsmede het kristalliseren van medicijnen in de poriën van het materiaal. Het materiaal hoeft niet beperkt te worden tot gebruik in de oogheelkunde, maar het materiaal kan tevens worden toegepast in andere applicaties, zoals intravasculair gebruik of voor medicijnafgifte aan de slijmvliezen of in het oor. Het materiaal biedt veel perspectief voor gecontroleerde afgifte van medicijnen door zijn hoge porositeit (tot wel $85 \%$ ). Het gebruik van dit materiaal als een platform voor medicijnafgifte is echter nog in ontwikkeling, en verdere in vivo experimenten moeten de aanwezigheid en veiligheid van dit materiaal aantonen, alsmede de medicijn concentraties in de verschillende weefsels in het oog. 


\section{List of Publications}

\section{Papers}

- $\quad$ Rachel T. Pijls, Hans, H.L. Hanssen, Rudy M.M.A. Nuijts, Leo H. Koole. Flexible coils with a drug-releasing hydrophilic coating: a new platform for controlled delivery of drugs to the eye? Biomedical Materials and Engineering 14 (4) (2004) 383-393.

- Rachel T. Pijls, Trudi Sonderkamp, Gert W. Daube, Ralph Krebber, Hans H.L. Hanssen, Rudy M.M.A. Nuijts, Leo H. Koole. Studies on a new device for drug delivery to the eye. European Journal of Pharmaceutics and Biopharmaceutics 59 (2) (2005) 283-288.

- $\quad$ Rachel T. Pijls, Sandra Lindemann, Rudy M.M.A. Nuijts, Gert W. Daube, Leo H. Koole. Pradofloxacin from the OphthaCoil: a new device for sustained delivery of drugs to the eye. Journal of Drug Delivery Science and Technology 17 (1) (2007) 87-91.

- $\quad$ Rachel T. Pijls, Lars P.J. Cruysberg, Hans H.L. Hanssen, Rudy M.M.A. Nuijts, Leo H. Koole. Capacity and tolerance of a new device for ocular drug delivery. International Journal of Pharmaceutics 341 (1-2) (2007) 152-161.

- $\quad$ Rachel T. Pijls, Peter P.K.H. Fransen, Hans H.L. Hanssen, Sandra Lindemann, Christiane Smetonat, Gert W. Daube, Leo H. Koole. The OphthaCoil for treatment of infections or pre-operative delivery of drugs to the eye. To be submitted.

- Rachel T. Pijls, Jérôme G.J.L. Lebouille, Aylvin A. Dias. Highly porous UHMWPE-films as a matrix for controlled drug delivery. To be submitted.

\section{Patents}

- Aylvin A. Dias, Leo H. Koole, Rachel T. Pijls. Coiled wire for the controlled release of drugs to the eye. WO2007/006427.

- Aylvin A. Dias, Rachel T. Pijls, Jérôme G.J.L. Lebouille. Porous matrix for delivery of a bioactive agent. Filing date: July 23, 2007.

- Aylvin A. Dias, Rachel T. Pijls, Jérôme G.J.L. Lebouille. Method for loading a bioactive agent in a porous matrix. Filing date: July 23, 2007.

\section{Abstracts}

- $\quad$ Rachel T. Pijls, Hans H.L. Hanssen, Rudy M.M.A. Nuijts, Gert W. Daube, Leo H. Koole. The OphthaCoil: a new ocular drug delivery system. Consecutive annual meetings of the Dutch Society for Biomaterials and Tissue Engineering (NBTE) Lunteren, the Netherlands (2003, 2004, 2006).

- $\quad$ Rachel T. Pijls, Hans H.L. Hanssen, Rudy M.M.A. Nuijts, Gert W. Daube, Leo H. Koole. The OphthaCoil: a new ocular drug delivery system. Integrated Biomedical Engineering (iBME) Papendal, the Netherlands, 2004, 2005. 
- $\quad$ Lars P.J. Cruysberg, Rachel T. Pijls, Hans H.L. Hanssen, Gert W. Daube, Rudy M.M.A. Nuijts, Leo H. Koole. Studies on a new device for controlled drug delivery to the eye. $199^{\text {th }}$ Annual Meeting of Nederlands Oogheelkundig Gezelschap (NOG) Maastricht, the Netherlands, 2005.

- $\quad$ Rachel T. Pijls, Hans H.L. Hanssen, Rudy M.M.A. Nuijts, Gert W. Daube, Leo H. Koole. In vivo tolerance and kinetics of a novel ocular drug delivery device. $9^{\text {th }}$ European Symposium on Controlled Drug Delivery (ESCDD) Noordwijk aan zee, the Netherlands. Also published in Journal of Controlled Release 116 (2) (2006) e47-49.

- $\quad$ Rachel T. Pijls, Hans H.L. Hanssen, Rudy M.M.A. Nuijts, Gert W. Daube, Leo H. Koole. Pradofloxacin release from the OphthaCoil: a new device for controlled delivery of drugs to the eye. National Biomedical Engineering Conference (BME) Egmond aan Zee, the Netherlands, 2007.

- $\quad$ Rachel T. Pijls, Lars P.J. Cruysberg, Hans H.L. Hanssen, Gert W. Daube, Rudy M.M.A. Nuijts, Leo H. Koole. In vivo release of pradofloxacin from a new ocular insert for controlled drug delivery. Annual Meeting of the Association for Research in Vision and Ophthalmology (ARVO) Fort Lauderdale, Florida, 2007.

- $\quad$ Rachel T. Pijls, Hans H.L. Hanssen, Gert W. Daube, Aylvin A. Dias, Leo H. Koole. Drug release from a hydrogel coated coil for controlled drug delivery to the eye. $7^{\text {th }}$ International Symposium on Frontiers in Biomedical Polymers (FBPS) Ghent, Belgium, 2007. 


\section{Curriculum Vitae}

Rachel Theodora Pijls was born on September $10^{\text {th }} 1980$ in Heerlen, the Netherlands. After graduating in 1998 from secondary school (VWO) at the Bernardinus College in Heerlen, she started to study Biomedical Engineering at the Eindhoven University of Technology. During this study, she came in contact with the biomaterials group of Prof. Dr. Ir. L.H. Koole in Maastricht and her interest in biomaterials science was initiated. In 2002, she did her graduation project in this group on the use of coated, coiled wires for the delivery of drugs to the eye. As part of this project, she worked for 3 months at the company Bayer HealthCare AG, in Monheim, Germany, under the supervision of Dr. G.W. Daube. There, she performed the first in vivo experiments with a developed drug delivery device, using Beagle dogs. In September 2003, she obtained her Masters degree in Biomedical Engineering. She decided to continue her research in the biomaterials group in Maastricht on the ocular drug delivery device. In November 2003, she started her PhD-project in collaboration with DSM Research in Geleen, the Netherlands, where she also did research on a porous material for the delivery of drugs. During her project she was under supervision of Prof. Dr. Ir. L.H. Koole. She worked in close collaboration with the Ophthalmology Department of the Academic Hospital of Maastricht and several companies in the Netherlands and Germany. The research she performed is described in this thesis.

Rachel Theodora Pijls werd geboren op 10 september 1980 in Heerlen. Na het behalen van haar VWO diploma aan het Bernardinus College in Heerlen, begon zij met de studie Biomedische Technologie aan de Technische Universiteit van Eindhoven. Tijdens deze studie, kwam zij in contact met de biomaterialen groep van Prof. Dr. Ir. L.H. Koole in Maastricht en haar interesse in biomaterialen was gewekt. In 2002 besloot zij haar afstudeerproject in deze groep te doen, aan het gebruik van gecoate spiralen voor de toediening van medicijnen aan het oog. In het kader van dit project heeft zij 3 maanden gewerkt bij Bayer HealthCare AG, in Monheim in Duitsland onder de supervisie van Dr. G.W. Daube. Hier deed ze in vivo experimenten met Beagle honden. In september 2003 haalde zij haar Ingenieurs-diploma (ir.) in de Biomedische Technologie. Zij besloot haar onderzoek over het toedienen van geneesmiddelen aan het oog in de biomaterialen groep in Maastricht te vervolgen. In november 2003 is zij haar AIO-project begonnen in samenwerking met DSM Research in Geleen, waar zij tevens onderzoek deed naar een poreus materiaal voor de toediening van geneesmiddelen. Tijdens haar project werkte zij onder supervisie van Prof. Dr. Ir. L.H. Koole. Zij heeft nauw samengewerkt met de afdeling Oogheelkunde van het Academisch Ziekenhuis in Maastricht en met verschillende bedrijven in Nederland en Duitsland. Het onderzoek dat zij uitgevoerd heeft, is beschreven in dit proefschrift. 


\section{Dankwoord}

Het einde van een tijdperk nadert. Vier jaar zijn voorbij gevlogen. Ik heb deze periode met heel veel plezier beleefd, zowel aan de Universiteit van Maastricht als bij DSM in Geleen. Ik wil daarom iedereen bedanken die gezorgd heeft dat deze tijd voor mij onvergetelijk was. Een aantal personen verdienen hierbij speciale aandacht:

Allereerst mijn promotor Leo Koole. Leo, je hebt mij veel kansen gegeven waarvan ik veel heb geleerd. Er was altijd een prettige en ontspannen sfeer in het lab en je vele ideeën en directe aanpak zijn bewonderenswaardig. Je was altijd zeer betrokken bij het onderzoek, en bij het welzijn van je medewerkers. Hartelijk bedankt voor je fijne begeleiding!

Mijn co-promotores Aylvin Dias en Rudy Nuijts. Aylvin, many thanks for giving me the opportunity to do my PhD project within DSM. You showed me how things work in the industry. Thanks a lot! Rudy, bedankt voor alle hulp bij het schrijven van de artikels en dit proefschrift. De relatie met medisch specialisten is voor ons werk van groot belang. Bedankt dat ik altijd bij je kon langskomen.

Alle leden van de beoordelingscommissie, met Prof. F. Hendrikse als voorzitter. Hartelijk dank voor het doornemen van dit proefschrift en de getoonde interesse in mijn onderzoek.

Natuurlijk wil ik ook mijn directe collega's in Maastricht bedanken. Allereerst, mijn kamergenootjes Erik en Tosca. Erik, bedankt voor de gezellige tijd op kantoor. Je bent inmiddels zelf gepromoveerd, en ik wens je veel succes met je verdere carrière. Tosca, bedankt voor alle steun in de afgelopen jaren, met name tijdens het schrijven van dit proefschrift. Ook wil ik Menno, Yvette en Ketie bedanken voor hun getoonde interesse in mijn onderzoek. Bedankt dat ik altijd bij jullie kon binnenlopen voor vragen en veel succes met jullie eigen onderzoeken.

Tevens wil ik mijn collega's bij DSM bedanken voor alle hulp en gezelligheid de afgelopen jaren. Bart, Mark en Marnix dank ik voor het mij wegwijs maken in het grote DSM wereldje. Tessa, Olga, Peter, Edith, Astrid, Jérôme, Audrey, Tristan, Wendy, Jeroen, Sebastian, Siete, George en Eva, allemaal bedankt voor de leuke, gezellige tijd bij DSM! Ook voor jullie geldt: veel succes met jullie eigen carrière.

Verder zijn er nog vele mensen die mij bij het onderzoek geholpen hebben. Allereerst, alle medewerkers van MCTec BV, bedankt voor jullie hulp bij het coaten en spiraliseren van de draden. Hans Hanssen, zonder Leo en jij was dit onderzoek niet van de grond gekomen. Bedankt dat ik altijd gebruik kon maken van de faciliteiten van je bedrijf. Ik wens jou, Leo en Mike veel succes met de verdere ontwikkeling van de OphthaCoil, die ik met veel interesse zal volgen.

I also would like to thank Bernhard Uihlein from EPflex (Dettingen, Germany) for taking care of sealing the ends of the OphthaCoil. Thank you very much for the fast response every time I needed you.

Special thanks to Dr. Gert Daube from Bayer HealthCare AG (Monheim, Germany). Rollo, many thanks for the opportunities you gave me at Bayer during my internship and for the 
pleasant collaboration afterwards! I always enjoyed the experiments we did together in Monheim and I appreciate the fact that you would like to take part in the committee during my defense. Also Sandra en Christiane, thanks a lot for all the help in the lab and with the dogs!

Lars Cruysberg en Sander Bootsma wil ik danken voor al hun hulp op de poli van Oogheelkunde. Lars, veel succes met de verdediging van je eigen proefschrift. Sander, jammer dat we de laatste klinische studie niet meer samen hebben kunnen uitvoeren. Ik wens jullie beide veel succes met jullie loopbaan.

Ook alle BMT-studenten die in ons lab in Maastricht hebben gewerkt in de tijd dat ik aio was, wil ik bedanken. Vooral Rob en Peter dank ik voor hun bijdrage aan dit onderzoek.

De mensen bij DSM Resolve dank ik voor alle metingen die zij hebben gedaan. Myraise van Stijn, bedankt voor je interesse in mijn onderzoek en alle SEM foto's van Solupor ${ }^{\circledR}$. Het was altijd prettig om even een praatje met je te maken. Sjoerd van der Wal, bedankt voor alle hulp met de HPLC, die niet altijd even perfect werkte.

Tevens een woord van dank aan: Trudie Sonderkamp, Math Hazen, Gijs Calis, Robbert van Sluis, Winand Habets, Pascale Vandevijver en Sarah Reiver. Ook alle aio's en medewerkers van Biochemie, bedankt voor de gezellige sfeer op de afdeling. Trees, bedankt voor de uitleg van alle administratieve dingen.

De meiden van mijn studietijd in Eindhoven: Tamara, Mirjam, Wilma, Kristie, Marcia en Silvia. Inmiddels zijn jullie al over het hele land, en zelfs naar Amerika, uitgespreid. Bedankt voor onze gezellige uitstapjes! Veel succes met jullie eigen onderzoeken en promoties.

De vrienden: Aniek en Dennis, Laura en Ralph, Ria en Maurice, Dorinda en Roland, Olga en Alessio, Tessa en Jeroen, bedankt voor alle ontspanning! Tessa en Jeroen, veel succes met jullie huis. Geniet ervan als het af is! Olga, je bent in korte tijd een heel goede vriendin geworden. Erg jammer dat je er op de dag zelf niet bij kunt zijn. Ik wens jou en Alessio veel geluk op jullie huwelijksreis!

Mijn paranimfen op deze dag zijn Tessa en Martine. Bedankt dat jullie mij op deze belangrijke dag willen steunen.

Ook alle familieleden en schoonfamilie wil ik danken voor de belangstelling in mijn onderzoek. Pap, mam en Lisanne, zonder jullie was ik nooit zover gekomen. Jullie hebben me de mogelijkheid gegeven om te studeren en mij vrij gelaten mijn eigen keuzes te maken. Bedankt voor jullie steun en vertrouwen.

Huib, jij hebt mij altijd gesteund door dik en dun. Ik ben blij dat jij op deze bijzondere dag aan mijn zijde staat.

\section{Rachel}

